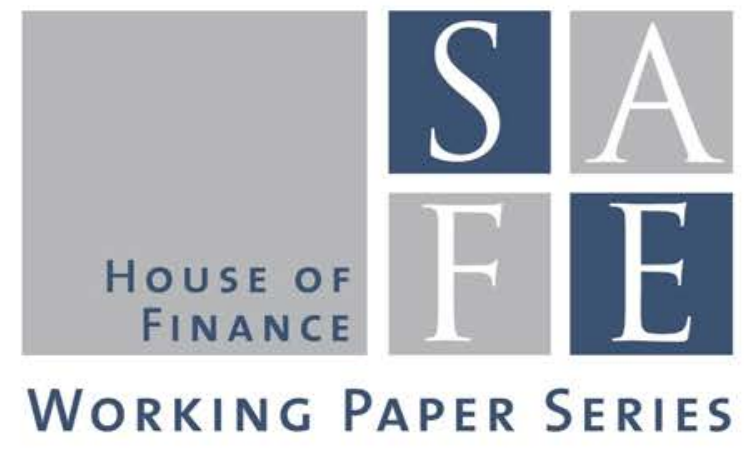

Giovani Bonaccolto - Massimiliano Caporin - Roberto Panzica

\title{
Estimation and model-based combination of causality networks
}

SAFE Working Paper No. 165

SAFE I Sustainable Architecture for Finance in Europe A cooperation of the Center for Financial Studies and Goethe University Frankfurt 


\section{Non-Technical Summary}

Statistical tests based on Granger causality are used in finance to detect the links among financial institutions that might be interpreted as channels along which shocks spread through the financial system. The links' structure can be formally defined as a network. Despite the growing interest in the financial network topic and the increasing understanding that there might be different channels over which financial contagion spreads, the literature on combinations of financial or economic networks is still very limited. In fact, the available competing approaches to estimate networks among financial institutions suggest the coexistence of different channels for the spread of risk. It is therefore of fundamental importance to allow for the possibility of combining those alternative risk spreading channels to obtain a more complete picture of risk propagation within the financial system. Furthermore, when focusing on the approaches for estimating financial company links, we believe that Granger causality should be contrasted with methods pointing in a more clear way to the risk dimension, for instance by detecting causality among quantiles. Therefore, we utilize parametric and notparametric approaches for the estimation on the networks based on quantile causality tests.

We show how to use a linear factor model as a device for estimating a combination of several networks that monitor the links across variables from different viewpoints, and we demonstrate that Granger causality test should be combined with quantile-based causality when the focus is on risk propagation. We empirically validate our two main proposals concerning the use of quantile causality to infer the network structure across a set of (financial) variables, and the model-based combination of causality networks. By using different datasets (US industrial portfolio returns, and a set of large banks and insurance companies), we first provide evidence of the different network structures that we can estimate from Granger causality and quantile causality. We then show how the networks differ across methods and over two different samples relating to the global financial crisis (2006-2008) and to the years 2011-2015. Our results suggest that quantile causality networks are denser than Granger causality networks, a finding of relevance to systemic risk interpretation, because a denser network is indicative of a much larger set of links, and thus explains a possibly greater systemic effect of shocks. 


\title{
Estimation and model-based combination of causality networks*
}

\author{
Giovani Bonaccolto ${ }^{\dagger} \quad$ Massimiliano Caporin ${ }^{\ddagger} \quad$ Roberto Panzica ${ }^{\S}$
}

This version: January 31, 2017

\begin{abstract}
Causality is a widely-used concept in theoretical and empirical economics. The recent financial economics literature has used Granger causality to detect the presence of contemporaneous links between financial institutions and, in turn, to obtain a network structure. Subsequent studies combined the estimated networks with traditional pricing or risk measurement models to improve their fit to empirical data. In this paper, we provide two contributions: we show how to use a linear factor model as a device for estimating a combination of several networks that monitor the links across variables from different viewpoints; and we demonstrate that Granger causality should be combined with quantile-based causality when the focus is on risk propagation. The empirical evidence supports the latter claim.
\end{abstract}

Keywords: Granger causality; quantile causality; multi-layer network; network combination J.E.L. codes: C58, C31, C32, G01

${ }^{*}$ We gratefully acknowledge research support from the Research Center SAFE, funded by the State of Hessen initiative for research LOEWE. We thank for the comments provided by the participants of the Computational and Financial Econometrics conference (CFE) held in Sevilla in December 2016, the Italian Conference of Econometrics and Empirical Economics held in Messina in January 2017.

${ }^{\dagger}$ University of Enna, Italy

${ }^{\ddagger}$ University of Padova, Italy; Corresponding author: Department of Statistical Sciences, Via C. Battisti 241, Padova, Italy, massimiliano.caporin@unipd.it

${ }^{\S}$ Goethe University Frankfurt, Germany 


\section{Introduction}

A growing strand of the financial economics literature has investigated the role of links between financial institutions, which serve as channels along which shocks spread through the financial system, so this strand of the financial economics literature is related to studies on financial contagion. The relationships between financial institutions may vary in nature and, as a consequence, the way in which we interpret these links may differ, depending on which type of relations are of interest to us, or which type of link we are monitoring or measuring. As examples, we cite the input-output relationship (where firms use the output of other companies as input, in their own production function), the ownership relationship (companies that hold assets of other companies), or the links measured on the grounds of stock market prices, such as causality-based connections.

The early literature on financial networks focused first on ascertaining the channel through which financial contagion was spreading within the financial system. The main objective was therefore to identify the networks by using appropriate criteria to detect the links between institutions. Following such an approach meant that the networks were, by definition, unique. Acemoglu et al. (2012) used a network structure based on input-output relationships to show that aggregate fluctuations may originate from microeconomic shocks to firms. Kelly et al. (2013), on the other hand, showed how stock firm volatility relates to customer-supplier connectedness. Billio et al. (2014) used contingent claim analysis and network measures to underscore the links between sovereign, banks and insurances. There is a constantly growing number of works proposing competing or alternative approaches for estimating the networks existing between groups of financial institutions, markets, countries and (not necessarily financial) assets. Among the many contributions in this area, we refer to Billio et al. (2012), Diebold and Yilmaz (2014, 2015), Hautsch et al. (2012, 2013, 2014, 2015), Barigozzi and Brownlees (2014), Ozdagli and Weber (2015), and Corsi et al. (2015), which have in common that they all refer to a financial or economic playground.

Despite the growing interest in the financial network topic, and the increasing understanding that there might be different channels over which financial contagion spreads, the literature on combinations of financial or economic networks is still very limited. In fact, the different approaches taken to estimating networks enable different potential channels for the spread of contagion or risk to come to light that might co-exist within the same financial system. It is therefore fundamentally important to allow for the possibility of combining them to obtain a more complete picture of risk spreading within a financial system.

This paper provides a possible solution based on a multiplex network, or a collection of networks (called layers) existing between a set of subjects, that we formally define in Section 2 , Ideally, the constituents of the multiplex network represent the outcomes of different approaches to the estimation or identification of links between the subjects analyzed. Postulating the existence of a multiplex network, we take a step forward and consider the combination of the information contained in the various layers of the network for the purpose of analyzing their impact within a financial economic model. In this case, we focus on the linear factor model augmented with network dependence introduced by Billio et al. (2016). We show how the model can be generalized to accommodate the presence of a multiplex network, and we provide a model 
parametrization that offers two useful interpretations of the models estimation output. First, the model parameters will enable the statistical significance of the information contained in each multiplex network layer to emerge. This will highlight the relevance of the various approaches to network measurement/identification within the model, with consequences on the financial interpretation associated with the model. These analyses might be relevant for policy purposes and market monitoring, as they may identify the main risk-spreading channel.

Second, the estimated model parameters will reveal a composite network obtained by combining the multiplex network layers. The model-based combination will thus provide an overall picture of the links existing between the variables analyzed, as measured using the different approaches (i.e. those behind the various layers of the network), as well as accounting for their respective relevance.

The model-based combination of networks is the first main contribution provided by the present paper. The second contribution that we make to the financial network literature concerns the estimation of networks. As previously mentioned, several authors have already discussed possible ways to obtain a network of financial assets. Among the various methods, the Granger causality approach of Billio et al. (2012) is taken as our starting point: Granger causality is used within a collection of bivariate Vector Auto Regressive (VAR) models to identify the statistically significant links across the modelled variables; then the network is used to identify the shock propagation channels and establish the systemic relevance of financial companies.

When focusing on the risk dimension, Granger causality within a VAR model detects mean causality whether it is on the financial contagion, or on the diffusion of systemic shocks, whereas we normally focus on variances or, more generally, on systemic and/or systematic risk measures. We consequently do not believe that using Granger causality will produce the most appropriate picture for interpreting the risk of the estimated financial networks. This prompts us to discuss a set of alternative approaches to estimating financial networks based on causality relationships that go beyond the classical Granger causality. We survey a collection of methods that have a common denominator: they all resort to the quantile regression approach of Koenker and Bassett (1978), in either a parametric or a non-parametric representation. Using these methods, we will show how we can identify causality among quantiles of the modelled variables. These quantile-based causality detection methods will lead to the estimation of causality networks mimicking the approach of Billio et al. (2012) but oriented towards a risk dimension. This is immediate when focusing on left quantiles of a random variable. As a further approach, we will also show that the non-parametric quantile causality test of Jeong et al. (2012) represents a further alternative to traditional Granger causality for building causality networks.

We stress that, by focusing on the quantiles of the modelled variables, we attach a clear risk interpretation to the estimated networks: on the one hand, the left quantiles are closely related to traditional risk measures such as the Value-at-Risk and the Expected Shortfall; on the other, the networks will monitor the spreading of shocks or the links between the modelled variables when they are in a high-risk state, and not when they are in a mean (or median) state, as captured by Granger causality. Our purpose is consistent with two other recent contributions, from Hong et al. (2009) and Corsi et al. (2015), that focus on causality among tail events. The Granger causality in risk in Hong et al. (2009), and also used by Corsi et al. (2015), is based on 
the occurrence of tail events and detects the possible causality among such events. We might associate the tail with a conditional quantile and estimate it with quantile regressions. We differ from the previous papers in that we focus on the causality within the assessment of the conditional quantiles, not on the occurrence of tail events. We thus consider the causality on the risk measures rather than the causality on the occurrence of extreme events.

We suggest different approaches to constructing causality networks, which involve monitoring the links across assets with views that might complement the (mean-based) Granger causality. These competing networks will represent the input for our model-based network combination. Using our model, we will be able to identify the relevance and the role of the various causality networks.

We empirically validate our two main proposals concerning the use of quantile causality to infer the network structure across a set of (financial) variables, and the model-based combination of causality networks. By using different datasets (US industrial portfolio returns, and a set of large banks and insurance companies), we first provide evidence of the different network structures that we can estimate from Granger causality and quantile causality. We show how the networks differ across methods and over two different samples relating to the global financial crisis (2006-2008) and to the years 2011-2015. Our results suggest that quantile causality networks are denser than Granger causality networks, a finding of relevance to systemic risk interpretation because a denser network is indicative of a much larger set of links, and thus a possibly greater systemic effect of shocks. The networks based on the non-parametric quantile causality test of Jeong et al. (2012) also have a clear core-periphery structure, which might help us to identify the more systemically relevant companies or sectors. Moreover, by resorting to the model-based combination approach, we show how different networks have a different impact and relevance on the various datasets, and we estimate a composite network. The model parameters indicate that quantile causality networks are much more relevant than Granger causality. In addition, the linear factor model augmented with a plurality of networks provides residuals that are much less correlated (on average) than traditional linear factor models, or the network augmented linear factor model of Billio et al. (2015), which is based on the Granger causality network alone. This latter finding might be implicated in explaining why the idiosyncratic risk is priced in the cross section. Since the quantile-based networks are the more relevant, it comes as no surprise that the composite network has a structure much closer to that of the quantile causality networks.

The paper proceeds as follows. Section 2 discusses the approach for obtaining a model-based composite network, extending the model of Billio et al. (2015). Section 3 presents the various approaches for estimating a causality network starting from the Granger causality method and then moving to quantile-based causality detection. Section 4 provides the empirical evidence with respect to both the estimation of quantile causality networks and the model-based combination of networks. This section also includes some robustness checks. Section 5 concludes. A Web Appendix accompanies the paper, which contains further tables and graphs, and some methodological notes. 


\section{Model-based network combination}

In this section, we introduce how we obtain a model-based combination of a collection of networks. At this stage, we assume that all the networks are available. We therefore do not estimate the structure of the various networks, but concentrate only on their optimal combination. We start with a formal definition of a simple network and of a multiplex or multilayer network.

A network or graph $G=(V, E)$ is a collection of vertexes $V$ and edges $E$, where the edges represent the links between the vertexes 1 with $E \subseteq(V \times V)$. Networks are generally represented by using the adjacency matrix $W$, a binary matrix where each element $w_{i, j}$ can take only two values, 1 and 0 . When $w_{i, j}$ is 1 , the node $j$ is linked to node $i$, with an information flow from $i$ to $j$. A value of zero identifies the absence of a link. We do not consider self-loops, so the diagonal of the $W$ matrix is identically equal to zero. If $W$ is symmetric, we associate with each node a measure called degree, that counts the number of edges for the node concerned. Note that, when focusing on $K$ assets, $W$ has a dimension $K \times K$.

The $W$ matrix might not be symmetric because we can have a link from $i$ to $j$, but the opposite might not hold. If the matrix $W$ is symmetric, the associated network is undirected as the information contained in the direction of the links becomes redundant; in this case, edges represent mutual relationships between nodes. If $W$ is not symmetric, the network is directed and there may be two edges between a pair of nodes; in this case, unlike the undirected case, we distinguish between indegree and outdegree. In particular, outdegree is the number of outgoing links starting from a given node, while indegree is the sum of the ingoing links on that node. If we relax the assumption of only two possible values for $w_{i, j}$ and allow for values within the (positive) real axis, the network becomes weighted (as opposed to unweighted) because the connection also has a size/relevance. In unweighted networks, each edge simply represents the presence of a connection, while in weighted networks edges also contain information on the strength of the relation between nodes.

As an example, the matrix in (1) represents the directed and unweighted network existing between a set of 5 nodes. Figure 1 provides a graphical representation of the network.

$$
W=\left[\begin{array}{lllll}
0 & 1 & 0 & 1 & 0 \\
1 & 0 & 1 & 0 & 0 \\
0 & 1 & 0 & 1 & 0 \\
0 & 0 & 1 & 0 & 1 \\
0 & 0 & 0 & 1 & 0
\end{array}\right]
$$

It is worth noting the parallelism between the adjacency matrix and the spatial (proximity) matrix used in spatial econometrics. The latter describes how the subjects of an analysis are spatially related. In such a different framework, the distance between subjects is (generally) measured on a physical (natural) playground, and connections correspond to neighborhood relationships. Therefore, if the element $w_{i, j}$ equals 1 , the element $j$ is a neighbor of the element $i$, following the spatial econometric nomenclature. The spatial matrices in the standard application are symmetric because neighboring relationships are symmetric. It is also common practice

\footnotetext{
${ }^{1}$ The terms vertex and nodes are equivalent, and both are used interchangeably in this work. In the same way, edges and links take on the same meaning.
} 


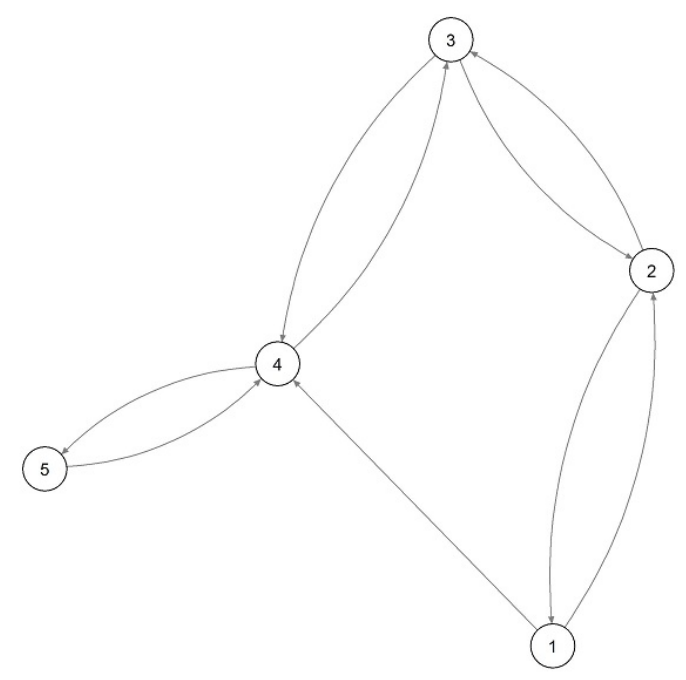

Figure 1: Network associated to the Matrix W

to focus on proximity matrices that are row-normalized. These matrices can therefore be interpreted as directed weighted networks, given that the symmetrical relations might become asymmetrical after normalization.

Within a financial framework, networks are generally directed and weighted when they are measured by means of balance sheet quantities. Billio et al. (2016) used BIS cross-holdings, Diebold and Yilmaz (2014) developed an approach based on variance decompositions of target series, Acemoglu et al. (2012) used the Input-Output matrix, as did Barigozzi and Brownlees (2014), Hautsch et al. (2014) and Hautsch et al. (2015). The network might also be directed and unweighted like those obtained using Granger causality in Billio et al. (2012), or Granger Causality in the tails (Corsi et al. 2015), or it might even be unweighted and undirected as in the economic sector case of Caporin and Paruolo (2015).

In the following, we assume that the strength of the edges is normalized at each node, as in a row normalization of the adjacency matrix $W$. We also assume that we are dealing with directed networks (the most general case that we could consider).

Assuming that we have $d$ different networks, the multiplex (or multilayer) network is a collection of the $d$ networks organized into $d$ different planes, or layers. In principle, the number of nodes needs not be the same across networks, so the total number of nodes in a multilayer network equals $\sum_{i=1}^{d} V_{i}$, where $V_{i}$ is the number of nodes or vertexes in the $i$-th layer.

Assuming that the graphs (associated with the networks) are simple (there are no loops), the maximum number of edges (connections between nodes) equals $\prod_{i=1}^{d} \frac{V_{i}\left(V_{i}-1\right)}{2}$, since connections across layers are also allowed.

In a financial framework, the multilayer network has an even simpler structure: the nodes are the same across layers because we have measures of connections over, say, the same financial institutions, but we use different approaches to estimate the networks. We also assume that there are no connections between layers, leaving us a collection of networks that are unrelated, but all referring to the same nodes. 
The literature on multiplex networks is relatively recent and, to the best of our knowledge, only a few papers combine networks in finance, focusing particularly on contagion and topological properties. Since institutions are linked in very different ways, it is useful to find a way to process all this information. Bargigli et al. (2015) analyzed the Italian interbank market using a multiplex network, distinguishing between five layers: unsecured overnight transactions, unsecured short-term transactions (up to 12 months), unsecured long-term transactions, secured short-term transactions and secured long-term transactions; they also considered the aggregated network, computed as the accumulation of all the layers.According to the authors, each layer had a different topological property and persistence over time and, among all the layers, only the overnight market was representative of the aggregate network.

Léon et al. (2014) studied the Colombian sovereign security market and built a multiplex network with three different layers corresponding to the three Colombian environments trading and registering sovereign securities. They found a strong nonlinear effect for the aggregate network value, attributable to the fact that the principal layer did not transfer its properties to the aggregate network or multiplex.

Montagna and Kok (2013) developed an agent-based multi-layered interbank network model using a sample of large EU banks, highlighting that there were non-negligible non-linearity effects influencing shock propagation to the individual banks. In other words, when different layers were considered simultaneously, the contagion effect was larger than the sum of the contagion-induced losses when the network layers were analyzed separately.

The present contribution aims to combine financial networks by building on a variant of a financial economic model, the linear factor model augmented with a spatial link following the approach of Billio et al. (2016).

The advantage of our model lies in that the combination of the various layers of the multiplex network is weighted with a set of coefficients appearing in the models parametrization. As well as representing the elements for a convex linear combination of the layers, the coefficients would consequently enable the significance and relevance of each layer to be assessed.

In the next subsection we briefly review the model of Billio et al. $(2016)$, and introduce the models generalization in the presence of a multilayer network. Then we discuss the approach used to derive a composite network from the models estimates.

\subsection{A linear factor model with multilayer spatial dependence}

To obtain a composite network, we generalize the model of Billio et al. (2016), a revisited multifactor model capable of taking into account the contemporaneous links between the assets (as measured by a network) together with the presence of common factors. In this model, the returns equation equals

$$
A\left(R_{t}-\mathbb{E}\left[R_{t}\right]\right)=\bar{\beta} F_{t}+\eta_{t}
$$

where $A$ is the matrix of the contemporaneous relationships between endogenous variables, i.e. the returns, coexisting with the exposure to a set of common factors $F_{t} ; R_{t}, \mathbb{E}\left[R_{t}\right]$ are, respectively, the asset returns and the expected asset returns, $\bar{\beta}$ is the structural asset exposure to the common factor and $\eta_{t}$ are the structural idiosyncratic residuals with a diagonal covariance 
matrix $\Sigma_{\eta}$.

The standard multifactor model can be seen as the reduced form of equation (2). Under the invertibility of matrix $A$, the model has the following reduced-form representation

$$
\begin{aligned}
R_{t} & =\mathbb{E}\left[R_{t}\right]+A^{-1} \bar{\beta} F_{t}+A^{-1} \eta_{t} \\
& =\mathbb{E}\left[R_{t}\right]+\beta^{\star} F_{t}+\epsilon_{t}^{\star}
\end{aligned}
$$

Starting from the reduced form, and in the presence of contemporaneous links across assets, Billio et al. (2016) make the point that: i) the reduced-form residuals $\epsilon_{t}^{\star}=A^{-1} \eta_{t}$ are crosscorrelated; and ii) the reduced-form betas, $\beta^{\star}=A^{-1} \bar{\beta}$, are a nonlinear function of the structural betas and of the contemporaneous relation matrix $A$.

The intuition of Billio et al. (2016) is to provide a structure for $A$ driven by the existence of a network representing the (contemporaneous) links across the assets. They therefore propose to make the matrix $A$ an affine function of a network, $A=(I-\rho W)$, where $\rho$ is a scalar parameter indicating the spatial dependence of the returns on the network, while $W$ is an exogenous spatial matrix, or adjacency matrix of a network. Such a choice also enables us to cope with the identification issues of the simultaneous equation system in (2).

Following Anselin (1988) and LeSage and Pace (2009), the model thus includes a spatial autoregressive component. The estimation of the spatial parameter $\rho$ follows from concentrated likelihood methods.

Billio et al. (2016) also allow for a more flexible parametrization of the $A$ matrix. In particular, they consider two relevant extensions:

- the use of asset-specific impacts from the network, which corresponds to a contemporaneous parameter matrix $A$ with equation-specific network impacts, namely $A=I-\mathcal{R} W$, where $\mathcal{R}=\operatorname{diag}\left(\rho_{1}, \rho_{2}, \ldots, \rho_{K}\right)$ is a diagonal matrix, and the coefficient $\rho_{i}$ represents the impact of the network on each asset; and

- the possibility of the spatial matrix, or network, varying in time, thus leading to $W_{t}$; this is particularly relevant in finance because $W$ is not related to a physical distance between subjects, but measures proximity or connections starting from variables liable to temporal differences.

We refer the reader to the paper by Billio et al. (2016) for further details on the models interpretation.

In the present paper, for the sake of simplicity, we assume the networks are time-invariant, though the approach that we introduce can easily be extended to the case of dynamic networks. In the presence of a multilayer network, we generalize the model of Billio et al. (2016) to accommodate the presence of several adjacency matrices.

In the spatial econometrics literature, we have some examples associated with the existence of richer spatial dynamics. In particular, Brandsma and Ketellapper (1979) propose a higher-order spatial autoregressive model, introducing two spatial matrices and consequently two spatial 
autocorrelation parameters: the first matrix based on the first-order neighbors, the second derived from the higher-order neighboring relations.

In the framework of Billio et al. (2016), the matrix of simultaneous relations $A$, in the presence of many networks, is easily generalized to

$$
A=I-\sum_{j=1}^{d} \rho_{j} W_{j}
$$

In equation (4), we have $d$ different scalar coefficients, $\rho_{j}$, capturing the impact of the network $W_{j}$ on the contemporaneous links across the assets.

Such a parametrization would give rise to a common impact of each network on the whole collection of modelled asset returns, so we propose to combine the existence of many networks with a restricted parametrization, accommodating the presence of the various networks, while also allowing for heterogeneous impacts of the networks on the assets. In detail, we suggest the following model specification:

$$
\left[I-\mathcal{R}\left(\sum_{j=1}^{d} \delta_{j} W_{j}\right)\right] R_{t}=A R_{t}=\mathbb{E}\left[R_{t}\right]+\beta F_{t}+\eta_{t}
$$

where $d$ is the number of layers or networks, $\mathcal{R}$ is a diagonal matrix, and the $\delta_{j}$ coefficients satisfy the following constraints:

$$
\sum_{j=1}^{m} \delta_{j}=1, \quad \delta_{j} \geq 0, j=1,2, \ldots m .
$$

The parameter $\delta_{j}$ controls the impact and role of each layer of the multiplex network, while the parameters in $\mathcal{R}$ determine the heterogeneous impact of the networks on the asset returns.

We suggest estimating the model using concentrated maximum likelihood, according to standard practice in spatial econometrics. If matrix $A$ is known, the factor loadings $\beta$ and the innovation covariance matrix $\Sigma_{\eta}$ can be obtained with traditional least square estimators. We can thus concentrate out the latter parameters and use concentrated maximum likelihood to estimate the parameters entering $A$. The maximization must account for the constraints that we impose on the $\delta_{j}$ parameters.

The model contains a sort of spatial autoregression, the structure of which is driven by several spatial proximity matrices. A standard requirement for estimations of spatial econometric models is the invertibility of matrix $A$. When the number of networks is restricted to one, or $d=1$, the non-singularity of $A^{-1}$ is ensured when $\lambda_{\text {min }}^{-1}<\rho<\lambda_{\text {max }}^{-1}$, where $\lambda_{\max }$ and $\lambda_{\min }$ are, respectively, the maximum and minimum eigenvalues of the spatial/adjacency matrix $W$.

In the presence of numerous proximity matrices, the non-singularity of $A$ might be addressed with analytical tools, but only in specific cases. For instance, Lee and Liu (2010) and Elhorst et al. (2012) discuss the case of two matrices $W$, referring to the estimation of a second-order spatial autoregressive model. These results are not easy to extend to our specification, however, we control for the invertibility of $A$ within the estimation step. 
The coexistence of many networks in the augmented linear factor model of Billio et al. (2016) has consequences on the identification of the models parameters too, particularly for those in $\mathcal{R}$.

The identification of the parameters entering the matrix $A$ is guaranteed by the constraints on the $\delta_{j}$ parameters, the row normalization in the $W_{j}$ matrices, and a further set of necessary (but simple and intuitive) conditions on the adjacency matrices:

i. $W_{j} \neq 0, \quad j=1,2, \ldots, d$;

ii. $W_{i} \neq W_{j}, \quad i, j=1,2, \ldots, d, \quad i \neq j$;

iii. $\rho\left(W_{i, .}\right)>0$ where $\rho(\mathcal{A})$ is the rank of matrix $\mathcal{A}, W_{i, .}=\left[\begin{array}{llll}\left.W_{1}^{\prime}\right|_{., i} & \left.W_{2}^{\prime}\right|_{., i} & \ldots & \left.W_{d}^{\prime}\right|_{., i}\end{array}\right]$ and $\left.W_{j}^{\prime}\right|_{., i}$ denotes the $i$-th row of $W_{j}$.

Condition [i] simply rules out the case of absence of edges between a collection of nodes. Condition [ii] excludes the case where two networks, measured by two different approaches are identical. If the latter case realize, we loose identification as the two networks are indistinguishable, and one of the two must be excluded from the analysis. The first two conditions are relevant for the identification of the $\delta_{j}$ parameters. Condition [iii] refers to the parameters in $\mathcal{R}$, and ensures that in each row of the combined network

$$
W^{\star}=\sum_{j=1}^{d} \delta_{j} W_{j} .
$$

we do have at least a non-null element. The parameter $\rho_{i, i}$ of the diagonal matrix $\mathcal{R}$ is identified if the $i$-th row of $W^{\star}$ has at least one non-null element. In fact, if a given row of $W^{\star}$ contains only null elements, the corresponding parameter in $\mathcal{R}$ is not identified. Such a case corresponds to asset $i$ having no spatial dependence on the other assets in the model, so we set the corresponding parameters in $\mathcal{R}$ to zero in the case of null rows in $W^{\star}$.

Finally, we note that the model is overidentified, given that the covariance matrix of the innovation, $\Sigma_{\eta}$, is diagonal, thus leaving space for any richer parametrizations in (5), with heterogeneous impacts on returns from groups of layers, for instance.

\subsection{Relevance and combination of networks}

The model offers two important insights. The first, and most relevant, concerns the feasibility of interpreting the summation of the adjacency matrices, matrix $W^{\star}$, as a new adjacency matrix representing a composite network. This is a direct consequence of the constraints on the coefficients leading to a convex linear combination of adjacency matrices, which are all rownormalized. The composite matrix will therefore also be a row-normalized matrix representing a combination of a collection of networks. The combination will also be directed and weighted because the initial networks are directed and weighted. The coefficients $\delta_{j}$ measure the relevance of each network layer in obtaining the composite network. The model thus generates both a composite network and an insight on the impact of each approach used to construct the various layers. 
Secondly, the parameters included in $\mathcal{R}$ represent the heterogeneous reaction of the assets to the composite network, thus making the multiple-network model comparable, in terms of its economic interpretation, to the network of Billio et al. (2016), but with the important difference that its underlying network is composite.

\section{Causality testing and estimating causality networks}

The concept of causality and the tools used to test for its existence date back to the seminal contributions of Granger $(1969,1980,1988)$. The purpose of his original contribution was to identify causal relationships in mean across economic variables, and his work attracted considerable interest in the econometrics literature, see Geweke (1984) and Hoover (2001). The concept was later extended to the analysis of causality among variances: Granger et al. (1986) provided a first definition of variance causality, while Comte and Lieberman (2000) extended and generalized the concept. On the testing side, Cheung and Ng (1996) proposed a test based on cross-correlations, Hafner (2003) introduced a procedure based on the likelihood of competing models, and Hafner and Herwartz (2008) presented a Wald-type approach.

More recently, there have been contributions on causality between two random variables that go well beyond the first- and second-order moments. We refer in particular to causality among quantiles such as the non-parametric test of Jeong et al. (2012) and the work of Lee and Yang (2014), and to the work of Candelon and Topkavi (2016) on causality in distributions.

Within a financial framework, the interest in causality and the use of Granger causality tests to estimate a network stems from the work done by Billio et al. (2012). The authors associated the statistical evidence of Granger causality in the mean with the existence of an edge connecting two assets (two nodes) of a financial network. In times of market turmoil, however, or when our interest lies in estimating systemic risk spreading after local or specific shocks, the causality in the mean might not be enough, and should be combined with a causality testing that covers the risk dimension.

In this section we provide a brief review of the Granger causality approach adopted by Billio et al. (2012), then suggest a number of competing methods we might consider for testing the existence of causality among quantiles of return distributions. As discussed in the introduction, the lower quantiles of returns acquire a more relevant role within a financial framework, because they correspond to Value-at-Risk levels. The availability of a number of competing methods for estimating causality networks enables the construction of a multiplex network, in which each layer is associated with a specific network. The multiplex network based on causality links can then be used for the model-based construction of composite networks, as discussed in the previous section.

The same criteria apply to the estimation of a single causality network, irrespective of the approach used to identify causality links. It is also important to note that we estimate a causality network starting from historical information on $K$ assets, generally focusing on asset returns.

The $K$ assets thus represent the nodes in the networks, and our purpose is to identify edges connecting each pair of nodes. If all assets are connected, and we exclude self-loops, we will have a total number of $K^{2}-K$ edges in which case the associated adjacency matrix is symmetric if the network is unweighted. 
In all the cases we discuss below, for any pair of assets $i$ and $j$, the existence of an edge connecting the nodes $i$ and $j$ with a specific direction (say, from $i$ to $j$ ) is established from a statistical test. The null hypothesis of the various tests corresponds to the absence of a causality link, while the null hypothesis is rejected in the presence of some form of causality. The type of causality detected clearly depends on the hypothesis tested.

For a given test statistic $\mathcal{M}$, and for any pair of assets $i$ and $j$, we define the edge from $i$ to $j$ as

$$
w_{i, j}=\left\{\begin{array}{cc}
0 & \mathcal{M} \text { null hypothesis rejected } \\
1 & \mathcal{M} \text { null hypothesis accepted }
\end{array}\right.
$$

where $w_{i, j}$ is the element in position $i, j$ within an adjacency matrix. Given the use of the test statistic $\mathcal{M}$, we denote the corresponding adjacency matrix as $W_{\mathcal{M}}$. The outcome of the test statistic, either rejecting or accepting the null, thus identifies the edges of a directed and unweighted network. We do not discuss here the possible generalization to a more flexible form of network construction that accounts for the strength of the causal relation too: such an extension is clearly possible but is left to future research. It should be noted that the networks will be row-normalized before they are used in the model presented in the previous section.

\subsection{Granger causality networks}

We start from the discussion of Granger causality testing, and the estimation of a Granger causality network in which case the framework for testing the presence of causality in mean between two variables $x_{t}$ and $y_{t}$ is the Vector Auto Regressive (VAR). We focus on the simplest VAR model with a single lag, that is

$$
\left[\begin{array}{l}
y_{t} \\
x_{t}
\end{array}\right]=\left[\begin{array}{ll}
a_{11} & a_{12} \\
a_{21} & a_{22}
\end{array}\right]\left[\begin{array}{l}
y_{t-1} \\
x_{t-1}
\end{array}\right]+\left[\begin{array}{l}
\varepsilon_{y, t} \\
\varepsilon_{x, t}
\end{array}\right]
$$

where we assume, for the sake of simplicity, that the innovation term is identically and independently distributed.

Testing for causality from $x_{t}$ to $y_{t}$ is tantamount to testing for the significance of the coefficient $a_{12}$, while the significance of $a_{21}$ provides information on the causality from $y_{t}$ to $x_{t}$. The framework can easily be generalized to the presence of more than one lag, in which case causality is associated with the null hypothesis of zero restrictions on a subset of the model parameters. Being obtained within a likelihood ratio framework, the test statistics have the usual Chi-square density.

The testing equation can also be extended by introducing further lagged or contemporaneous explanatory variables that affect both the dependent variables. This would bring to light a form of common behavior that might hide or distort the identification of causal relations. Notably, by replacing the series levels with their squares, or by focusing on realized volatility sequences, the Granger causality framework also enables us to test for causality between risk measures.

Billio et al. (2012) were the first to adopt Granger causality to estimate a relationship-based network among financial assets. The connections between nodes in their network denote the presence of a causality relation in the Granger sense. 
The approach used by Billio et al. (2012) starts from a collection of series of $K$ asset returns over a daily sample of size $T$, which we denote by $R_{i, t}$, with $i=1,2, \ldots, N$ and $t=1,2, \ldots T$. Returns are first filtered by means of a $\operatorname{GARCH}(1,1)$ model to eliminate the well-known heteroskedastic behavior. The authors thus estimate

$$
R_{i, t}=\mu_{i}+\eta_{i, t} \quad i=1,2, \ldots N
$$

where $\mu_{i}$ is the unconditional mean and $\eta_{i, t}$ is the innovation for asset $i$. Following the standard literature, $\eta_{i t}=\sigma_{i t} \epsilon_{i t}$, where $\sigma_{t}$ is the conditional standard deviation. The conditional variance follows a simple $\operatorname{GARCH}(1,1)$ process

$$
\sigma_{i, t}^{2}=\omega_{i}+\alpha_{i} \eta_{i, t-1}^{2}+\beta_{i} \sigma_{i, t-1}^{2} \quad i=1,2, \ldots N
$$

with $\omega_{i} \geq 0, \alpha_{i, 1} \geq 0, \beta_{i} \geq 0$, and $\alpha_{i}+\beta_{i}<1$. As usual in the GARCH literature, $\varepsilon_{i, t}$ is assumed to be a sequence of i.i.d random variables with zero mean and unit variance. After the model estimation, we can therefore obtain the so-called standardized residuals $\varepsilon_{i, t}=\frac{\eta_{i, t}}{\sigma_{i, t}}$.

The next step develops Granger's causality test on each pair of standardized asset residuals, but the simple framework of equation (9) might lead to the detection of spurious causality. In fact, if we take three assets, $i, j$ and $z$, and we have $i \rightarrow_{G} j$ (the series $i$ causes the series $j$ in the sense of Granger causality) and $j \rightarrow_{G} z$, then by using the standard Granger causality test we might also find that $i \rightarrow_{G} z$, but such a causality could be a by-product of the presence of $j$, and not a real direct causality impact. To control for such effects, Billio et al. (2012) augment the model in (9) with a so-called background series and/or a set of common factors, leading to the following model

$$
\left[\begin{array}{c}
\varepsilon_{i, t} \\
\varepsilon_{j, t}
\end{array}\right]=\left[\begin{array}{ll}
a_{11} & a_{12} \\
a_{21} & a_{22}
\end{array}\right]\left[\begin{array}{l}
\varepsilon_{i, t-1} \\
\varepsilon_{j, t-1}
\end{array}\right]+\left[\begin{array}{l}
b_{1} \\
b_{2}
\end{array}\right] \varepsilon_{z, t-1}+\beta F_{t-1}+\left[\begin{array}{c}
\varphi_{i, t} \\
\varphi_{j, t}
\end{array}\right]
$$

where $F_{t}$ might contain common factors, and with $i, j, z=1,2, \ldots N$, and $i \neq j \neq z$. Obviously, we have $N-2$ possible background series for each pair of GARCH standardized residuals $i, j$.

The Granger causality test must therefore discriminate between the various choices of $z$, conditional to the possible presence of common factors $F_{t}$. The approach of Billio et al. (2012) made use of information criteria to specify the VAR lag structure and select the background series. Once the background series had been selected, the authors ran a set of Granger causality tests including/excluding the background series and/or the common factors, and selected the test outcome with the highest p-value (of the Granger's causality test) to produce more robust results.

We point out that the approach is computationally intensive because detecting the causality from $i$ to $j$, for a specific choice of $i$ and $j$, involves estimating $N-2$ bivariate systems to select the background series, and then further estimates to perform the causality testing.

For each pair of GARCH standardized residuals $i$ and $j$, the test enables us to check for the presence of causality from $i$ to $j$, with a decision rule associated with the above-described procedure. The decision rule provides for just two outcomes, a 0 or a 1 : if causality is present, 
the approach of Billio et al. (2012) leads to the creation of a connection from node $i$ to node $j$. The adjacency matrix gives rise to a directed and unweighted network. Note that, in the empirical analyses, we obtain the network following the approach of Billio et al. (2012) and, before estimating our model, we proceed with a row normalization of the network.

\subsection{Quantile causality: a baseline case}

Granger causality tests focus on the possible presence of causality relations affecting the mean dynamic evolution of $x_{t}$ and $y_{t}$. As noted earlier, this approach might easily be extended to testing causality among higher-order moments.

Despite the usefulness and simplicity of this approach, focusing on the moments alone might prove too restrictive, since it could preclude or limit the possibility of studying the causal relations between $x_{t}$ and $y_{t}$ in specific regions of their distributions, or the risk measures associated with the distributions of the two variables, such as quantiles or conditional moments.

For instance, there might be situations where $x_{t}$ and $y_{t}$ are not linked by causal relations in the center of their distributions, or in the mean, but they might still be strongly dependent on one another in the tails of their distributions. This phenomenon could be crucial in the context of financial returns (given that their distributions are typically characterized by fat tails (Cont, 2001)), or when we are interested in the existence of causal relations during periods of market turmoil (when both the variables analyzed take values in their tails), or during a negative period for the target variable (when only the dependent variable takes values in its tail). There might also be more interesting cases in which the causal relations are asymmetrical between the left and right tails. The classical Granger's test and its baseline variants are obviously unable to detect such structural features.

This shortcoming can be overcome by building causality tests based on the quantile regression method introduced by Koenker and Bassett (1978). In such a framework, we postulate the existence of a linear relationship between the quantile of a target variable and the values taken by an explanatory variable. If we deal with two variables, we thus have two equations, and the bivariate system becomes:

$$
\begin{aligned}
& Q_{y_{t}}(\tau)=\beta_{0,1}(\tau)+\beta_{1,1}(\tau) y_{t-1}+\beta_{2,1}(\tau) x_{t-1} \\
& Q_{x_{t}}(\theta)=\beta_{0,2}(\theta)+\beta_{1,2}(\theta) y_{t-1}+\beta_{2,2}(\theta) x_{t-1}
\end{aligned}
$$

where $Q_{y_{t}}(\tau)$ and $Q_{x_{t}}(\theta)$ denote the $\tau$-th and $\theta$-th conditional quantiles of $y_{t}$ and $x_{t}$, respectively. As in the idea behind Granger causality, testing for causality from $x_{t}$ to the $\tau$-th conditional quantile of $y_{t}$ involves testing the significance of the coefficient $\beta_{2,1}(\tau)$. Likewise, testing for causality from $y_{t}$ to the $\theta$-th conditional quantile of $x_{t}$ involves testing the significance of $\beta_{1,2}(\theta)$.

Unlike the Granger causality testing approach, the estimation of the conditional quantiles is based on the marginal model for each target variable. We therefore test for causality from $x_{t}$ to $y_{t}$, and from $y_{t}$ to $x_{t}$ using two different models (i.e. two different conditional quantile linear models, one for each target variables). A recently-developed system approach is also viable, as discussed later on.

Financial variables are often highly correlated, due mainly to their sensitivity to market trends and shocks. The influence of these common factors can be isolated by including one or 
more control variables reproducing the market movements in the bivariate system (13). If we let $\mathbf{F}_{t}$ be the vector of these common factor variables observed in $t$, we rewrite the (13) as

$$
\begin{aligned}
& Q_{y_{t}}(\tau)=\beta_{0,1}(\tau)+\beta_{1,1}(\tau) y_{t-1}+\beta_{2,1}(\tau) x_{t-1}+\gamma_{1} F_{t-1}^{\prime} \\
& Q_{x_{t}}(\theta)=\beta_{0,2}(\theta)+\beta_{1,2}(\theta) y_{t-1}+\beta_{2,2}(\theta) x_{t-1}+\gamma_{2} F_{t-1}^{\prime}
\end{aligned}
$$

The latter conditional quantiles thus mimic the presence of common factors used in the Billio et al. (2012) approach. Similarly, we can introduce a further background variable, and increase the lag structure within the conditional quantile specification. Note that we could introduce lags for both the conditioning and the modelled variables.

If we focus on the conditional quantile for $x_{t}$ (similarly for $y_{t}$ ), with or without additional control or background variables, the detection of causality is associated with the presence of a significant impact of $y_{t-1}$ (or all lags if the models structure is more complex) on the conditional quantile of $x_{t}$.

Within a quantile regression framework, we can also assess the causality impact of $x_{t}$ by comparing the first equation in (13) with its restricted version:

$$
Q_{y_{t}}^{(r)}(\tau)=\beta_{0,1}^{(r)}(\tau)+\beta_{1,1}^{(r)}(\tau) y_{t-1},
$$

on the basis of the testing procedure proposed by Koenker and Bassett (1982). The test verifies the null hypothesis that the additional variable in the first equation belonging to the system (13), i.e. $x_{t-1}$, does not improve on the goodness-of-fit achieved with the restricted model given in (15). If the null hypothesis of the test, which reads like the F-test, is rejected at a given significance level, then there is evidence of causality from $x_{t}$ to the $\tau$-th conditional quantile of $y_{t}$. The same method could likewise be applied to test the causality relations from $y_{t}$ to the $\theta$-th conditional quantile of $x_{t}$.

Finally, we note that the quantile causality network is specific to the $\tau$ quantile used to test the link between $x_{t}$ and $y_{t}$. The above-outlined method might therefore generate a collection of quantile causality networks. When the focus is on the risk, however, our interest lies in the left tail of the distribution, and therefore on small values of $\tau$, ideally between $1 \%$ and $10 \%$.

Using conditional quantile causality testing based on an assessment of the significance of the coefficients, we thus obtain the quantile causality network at quantile $\tau$. We stress that the estimated network depends on the chosen quantile, so we can estimate a collection of quantilebased causality networks.

\subsection{Quantile-on-quantile causality}

So far, we have considered the causal impact of one variable on another, focusing either on moments (within a Granger causality framework) or on conditional quantiles. We can take the analysis further by directly linking the quantiles of both the causal and the dependent variables. In other words, our aim is to check whether the $\theta$-th quantile of $x_{t}$ causes the $\tau$-th quantile of $y_{t}$, and vice versa, for $\theta$ equaling or differing from $\tau$. In this way, we can test whether the power of $x_{t}$ in causing the $\tau$-th quantile of $y_{t}$ changes over $\theta \in(0,1)$.

From a different viewpoint, while in traditional quantile regression we analyze the possible 
impact of the values taken by $x_{t-1}$ (across its whole density) on the conditional quantile of $y_{t}$, for instance, we are interested here in testing the existence of causality when we restrict our attention to a neighborhood of a quantile of $x_{t-1}$. This would enable us to seek causal relations when both the analyzed variables take values in the respective tails, for example.

Two different approaches can be used for this purpose. The first is based on the method introduced by Sim and Zhou (2015), who proposed a modified version of quantile regression in which observations (and then the corresponding loss function) are weighted using a kernel function. In particular, Sim and Zhou (2015) use kernel-based weights to estimate the relations in quantiles between oil prices and stock returns. In our framework, following Sim and Zhou (2015), the conditional quantiles in 13 are rewritten, respectively, as follows:

$$
\begin{aligned}
Q_{y_{t}}(\tau, \theta) & =\beta_{0,1}(\tau, \theta)+\beta_{1,1}(\tau, \theta) y_{t-1}+\beta_{2,1}(\tau, \theta) x_{t-1} \\
Q_{x_{t}}(\theta, \tau) & =\beta_{0,2}(\theta, \tau)+\beta_{1,2}(\theta, \tau) y_{t-1}+\beta_{2,2}(\theta, \tau) x_{t-1}
\end{aligned}
$$

where the only difference lies in the fact that the parameters have both $\tau$ and $\theta$ subscripts. In fact, the estimated parameters depend on the quantile levels of both $y_{t}$ and $x_{t}$. Despite the similarities in the form, the equations in system (16) differ from those in 13 in the manner in which the unknown parameters are estimated. Notably, the coefficients belonging to the first equation of the system (16) come from the following minimization problem:

$$
\begin{gathered}
\min _{\beta_{0,1}(\tau, \theta), \beta_{1,1}(\tau, \theta), \beta_{2,1}(\tau, \theta)} \sum_{t=1}^{T} \rho_{\tau}\left[y_{t}-\beta_{0,1}(\tau, \theta)-\beta_{1,1}(\tau, \theta) y_{t-1}-\beta_{2,1}(\tau, \theta) x_{t-1}\right] * \\
* K\left(\frac{F_{T}\left(x_{t}\right)-\theta}{h}\right),
\end{gathered}
$$

where $\rho_{\tau}(e)=e\left(\tau-\mathbf{I}_{\{e<0\}}\right)$ is the asymmetric loss function on the basis of the quantile regression method introduced by Koenker and Bassett (1978), and $\mathbf{I}_{\{\cdot\}}$ is the indicator function, taking a value of 1 if the condition in $\{\cdot\}$ is true, and a value of 0 otherwise. The difference with respect to the classical quantile regression of Koenker and Bassett (1978) lies in $K(\cdot)$, i.e. the kernel function, with bandwidth $h$, where $F_{T}\left(x_{t}\right)=T^{-1} \sum_{k=1}^{T} \mathbf{I}_{\left\{x_{k}<x_{t}\right\}}$, to focus on the impact of $x_{t}$ in the neighborhood of its $\theta$-th quantile. The parameters in the second equation of the system (16) are likewise estimated from

$$
\begin{gathered}
\min _{\beta_{0,2}(\theta, \tau), \beta_{1,2}(\theta, \tau), \beta_{2,2}(\theta, \tau)} \sum_{t=1}^{T} \rho_{\theta}\left[x_{t}-\beta_{0,2}(\theta, \tau)-\beta_{1,2}(\theta, \tau) y_{t-1}-\beta_{2,2}(\theta, \tau) x_{t-1}\right] * \\
* K\left(\frac{F_{T}\left(y_{t}\right)-\tau}{h}\right) .
\end{gathered}
$$

If we remove the kernel function, the parameters no longer depend on $\theta$, and we return to the traditional quantile regression estimator. As discussed in the previous section, we can generalize the conditional quantiles in (16) by adding common factors and background variables. Notably, what Sim and Zhou (2015) call the quantile-on-quantile approach corresponds to a nonparametric quantile regression where the knots used to obtain the local quantiles are fixed at specific quantiles of the dependent variable (Koenker, 2005).

The construction of a quantile-causality network by following the quantile-on-quantile ap- 
proach of Sim and Zhou (2015) builds on the significance of the coefficients associated with the impact of the covariates quantiles. In particular, focusing on $x_{t}$, the existence of causality from the $\theta$ quantile of $y_{t}$ to the $\tau$ quantile of $x_{t}$ depends on the coefficient $\beta_{1,2}(\theta, \tau)$. If the conditional quantiles have a more complex structure, including several lags, the number of coefficients to be tested naturally increases. When accounting for the presence of common factors and background variables, we can again follow a procedure similar to that of Billio et al. (2012).

When estimating the quantile causality network by means of the quantile-on-quantile method, the estimated network depends on two quantile levels: one referring to the dependent variable and the other to the explanatory variable. As in the previous section, if we are interested in monitoring the causality networks during periods of financial turmoil, both quantiles would be placed in the range of $1 \%-10 \%$.

We note that such an approach has some similarities with the VAR for VaR model introduced by White et al. (2015), which, in a multivariate framework, estimates the dependence across quantiles for a collection of series, also accounting for the possible presence of an auto-regressive structure, in the spirit of the CAViaR by Engle and Manganelli (2004). Clearly, the quantileon-quantile dependence might be seen as a special case.

\subsection{Quantile causality: a nonparametric test}

The previous quantile regression-based approaches for detecting causality have a relevant feature in common, that is their parametric nature. In this section, we refer to another approach that is non-parametric. Non-parametric techniques offer the important advantage of requiring no particular assumption concerning the distributions for the processes underlying the variables of interest. We refer here to the nonparametric testing procedure proposed by Jeong et al. (2012).

First, we assume that the conditional variable is $x_{t}$, and we define the following vectors: $\mathcal{Y}_{t-1} \equiv\left(y_{t-1}, \ldots, y_{t-p}\right), \mathcal{X}_{t-1} \equiv\left(x_{t-1}, \ldots, x_{t-q}\right), \mathcal{Z}_{t-1} \equiv\left(y_{t-1}, \ldots, y_{t-p}, x_{t-1}, \ldots, x_{t-q}\right)$, for $(p, q)>$ 1. Note that the vectors refer to the lags of one or both the variables of interest. Further, $F_{y_{t} \mid \mathcal{Z}_{t-1}}\left(y_{t} \mid \mathcal{Z}_{t-1}\right)$ and $F_{y_{t}} \mid \mathcal{Y}_{t-1}\left(y_{t} \mid \mathcal{Y}_{t-1}\right)$ denote the distributions of $y_{t}$, conditional on $\mathcal{Z}_{t-1}$ and $\mathcal{Y}_{t-1}$, respectively; the distribution of $y_{t}$ is assumed to be absolutely continuous in $y$ for almost all $\nu=(\mathcal{Y}, \mathcal{Z})$. For the sake of simplicity, we denote the $\tau$-th quantile of $y_{t}$ conditional on $\mathcal{Z}_{t-1}$ as $Q_{\tau}\left(\mathcal{Z}_{t-1}\right)$ and the $\tau$-th quantile of $y_{t}$ conditional on $\mathcal{Y}_{t-1}$ as $Q_{\tau}\left(\mathcal{Y}_{t-1}\right)$.

The definition of causality in quantiles in Jeong et al. (2012) focuses on the conditional quantiles of the series densities: $x_{t}$ does not cause $y_{t}$ in its $\tau$-th quantile, with respect to $\mathcal{Z}_{t-1}$, if $Q_{\tau}\left(\mathcal{Z}_{t-1}\right)=Q_{\tau}\left(\mathcal{Y}_{t-1}\right)$; conversely, $x_{t}$ causes $y_{t}$ in its $\tau$-th quantile, with respect to $\mathcal{Z}_{t-1}$, if $Q_{\tau}\left(\mathcal{Z}_{t-1}\right) \neq Q_{\tau}\left(\mathcal{Y}_{t-1}\right)$. From the previous definition, the hypotheses to be tested read as follows:

$$
\left\{\begin{array}{l}
H_{0}: P\left[F_{y_{t} \mid \mathcal{Z}_{t-1}}\left(Q_{\tau}\left(\mathcal{Y}_{t-1}\right) \mid \mathcal{Z}_{t-1}\right)=\tau\right]=1 \\
H_{1}: P\left[F_{y_{t} \mid \mathcal{Z}_{t-1}}\left(Q_{\tau}\left(\mathcal{Y}_{t-1}\right) \mid \mathcal{Z}_{t-1}\right)=\tau\right]<1
\end{array}\right.
$$

The non-parametric test developed by Jeong et al. (2012) is based on a measure of distance defined as:

$$
J_{T}=\mathbb{E}\left[\left[F_{y_{t} \mid \mathcal{Z}_{t-1}}\left(Q_{\tau}\left(\mathcal{Y}_{t-1}\right) \mid \mathcal{Z}_{t-1}\right)-\tau\right]^{2} g_{\mathcal{Z}_{t-1}}\left(\mathcal{Z}_{t-1}\right)\right]
$$


where $g_{\mathcal{Z}_{t-1}}\left(\mathcal{Z}_{t-1}\right)$ is the marginal density function of $\mathcal{Z}_{t-1}$. $J_{T}$ is estimated using the feasible kernel-based estimator:

$$
\widehat{J}_{T}=\frac{1}{T(T-1) h^{m}} \sum_{t=1}^{T} \sum_{s \neq t} K\left(\frac{\mathcal{Z}_{t-1}-\mathcal{Z}_{s-1}}{h}\right) \tilde{\epsilon}_{t} \tilde{\epsilon}_{s},
$$

where $m=p+q, K(\cdot)$ is the kernel function with bandwidth $h, \tilde{\epsilon}_{t}=\mathbf{I}_{\left\{y_{t} \leq \widetilde{Q}_{\tau}\left(\mathcal{Y}_{t-1}\right)\right\}}-\tau, \mathbf{I}_{\{\cdot\}}$ being the indicator function that takes a value of 1 if the condition in $\{\cdot\}$ is true, and zero otherwise. In Jeong et al. $(2012), \widetilde{Q}_{\tau}\left(\mathcal{Y}_{t-1}\right) \equiv \widetilde{F}_{y_{t} \mid \mathcal{Y}_{t-1}}^{-1}\left(\tau \mid \mathcal{Y}_{t-1}\right)$, where

$$
\widetilde{F}_{y_{t} \mid \mathcal{Y}_{t-1}}\left(y_{t} \mid \mathcal{Y}_{t-1}\right)=\frac{\sum_{s \neq t} C_{t-1, s-1} \mathbf{1}_{\left\{y_{s} \leq y_{t}\right\}}}{\sum_{s \neq t} C_{t-1, s-1}}
$$

is the Nadaraya-Watson kernel estimator of $F_{y_{t}} \mid \mathcal{Y}_{t-1}\left(y_{t} \mid \mathcal{Y}_{t-1}\right)$, with the kernel function $C_{t-1, s-1}=$ $C\left(\mathcal{Y}_{t-1}-\mathcal{Y}_{s-1}\right) / a$, and $a$ is the bandwidth.

Under a set of precise assumptions, Jeong et al. (2012) derived the limiting distribution for the test statistic

$$
T h^{m / 2} \widehat{J}_{T} \stackrel{L}{\rightarrow} \mathcal{N}\left(0, \sigma_{0}^{2}\right)
$$

where

$$
\sigma_{0}^{2}=2 \mathbb{E}\left[\sigma_{\epsilon}^{4}\left(\mathcal{Z}_{t-1}\right) g_{\mathcal{Z}_{t-1}}\left(\mathcal{Z}_{t-1}\right)\right]\left(\int K^{2}(u) d u\right)
$$

and $\sigma_{\epsilon}^{2}\left(\mathcal{Z}_{t-1}\right)=\tau(1-\tau)$.

The unknown parameter $\sigma_{0}^{2}$ is estimated as

$$
\widehat{\sigma}_{0}^{2}=\frac{2 \tau^{2}(1-\tau)^{2}}{T(T-1) h^{m}} \sum_{s \neq t} K^{2}\left(\frac{\mathcal{Z}_{t-1}-\mathcal{Z}_{s-1}}{h}\right) .
$$

Then, after estimating all the quantities of interest, the standardized statistic can be computed:

$$
\widehat{J}_{T}^{*}=\frac{T h^{m / 2} \widehat{J}_{T}}{\widehat{\sigma}_{0}} .
$$

The above-described method could likewise be applied to test the causality impact of $y_{t}$ on the quantiles of $x_{t}$, simply by inverting the roles of $x_{t}$ and $y_{t}$.

If we detect quantile causality using the non-parametric test of Jeong et al. (2012), then we have a dependence on the chosen quantile, as in the previous quantile-based causality cases. To keep the focus on the risk side, we suggest using quantile levels in the range 1\%-10\%.

\section{Empirical examples}

\subsection{Data description}

The empirical analyses are conducted on three different datasets: the first concerns the 48 industry portfolios on the Kenneth R. French website; the second includes the 25 US banks with 
the greatest market value; the third the 25 US insurance firms with the highest market value 2 Using the three datasets, we can test for the presence of causality links from a general economic standpoint, or by looking at the companies that were mostly affected by the recent financial crisis.

For the three datasets, we use the daily returns over two different samples. The first spans the open-market business days between 3 January 2006 and 31 December 2008. The second covers the period between 3 January 2011 and 31 December 2015. The former is characterized by 755 daily returns for each time series, while the latter records 1258 observations for each industry portfolio or firm. The two samples behave very differently, since the first includes the years of the global financial crisis, while the second covers a period of upward trend in the financial market, excluding the initial recovery period after the crisis. Our purpose is to test whether there is a different dependence structure among the variables during periods of market distress vis-à-vis periods with a positive market trend. We proceed with our estimation of causality networks by focusing on the daily returns.

Just as we selected the 25 US banks and the 25 US insurance firms with the highest market value as at the beginning of 2006, for the first time interval considered, the choice of banks and insurance companies to consider in the second period was based on their market value as at the beginning of 2011. This means that most, but not all of these financial institutions are included in both of the samples. Appendix A contains a list of the selected banks and insurance companies. We stress that our objective is to obtain an overview of the changes occurring in the links across nodes (banks, insurance companies, or economic sectors) when we change the approach used to ascertain the existence of nodes, switching from Granger causality to quantilebased causality, to arrive at the optimal combination of competing available networks.

Tables B.9. B.10 in Appendix B show the main descriptive statistics computed for the banks in the first and second time intervals, respectively; Tables B.11 B.12 concern the insurance companies and the statistics for the 48 industry portfolios are given in Tables B.13 B.14. In all cases, the average returns are lower in 2006-2008 than in 2011-2015. This was expected and is due to the effects of the global financial crisis. The first time interval is also characterized by a greater uncertainty, averaging higher standard deviations and interquartile ranges; this was also expected. The risk affecting the years 2006-2008 is also apparent from the corresponding minimum and maximum returns. The distributions of these returns are fat-tailed (especially in the first period), consistently with the stylized facts highlighted by Cont (2001). The distributions are also highly skewed. Finally, we used the Ljung-Box test for mean serial dependence on both the returns and the squared returns, using 1,5 and 10 lags. As expected, the outcomes show no serial dependence on returns, while squared returns (a proxy of variances) are highly correlated, suggesting the presence of heteroskedasticity. In short, the descriptive analysis shows significant differences between the years 2006-2008 and 2011-2015, that are clearly a consequence of the turmoil affecting the US economy during the former period. We now take a closer look at the dependence across companies or economic sectors by focusing on causality analyses.

\footnotetext{
${ }^{2}$ The series of industry portfolios are available at http://mba.tuck.dartmouth.edu/pages/faculty/ken.french/, the data on the banks and insurance firms were obtained from Thomson Reuters Datastream.
} 


\subsection{Granger and quantile causality networks}

In building the causality networks, we extend the existing literature by combining the classic Granger testing approach with methods based on analyses of dependence across quantiles, as described in Section 3.1.

The methods based on quantile regression and quantile causality depend on the quantile at which the analysis is performed, so we must first specify which quantiles we use to conduct the dependence or causality analysis. Our analysis illustrates the changes occurring in the causality networks when we switch from Granger causality to quantile causality, without identifying the optimal quantile over which to measure quantile causality. We therefore decided to fix the quantiles of interest a priori: we chose three quantiles, $0.1,0.5$ and 0.9 , corresponding to the tails and the center of the distributions of the returns. Note that, when considering the approach of Sim and Zhou (2015), where we have quantile causality depending on two different quantiles, we set both quantiles at the same level.

For the estimation of quantile regressions, we assess the standard errors of the parameters with the $x y$-pair bootstrap method, which provides accurate results without assuming any particular distribution for the error term. When a kernel function is involved, we set it to the Gaussian one. We set the confidence level at $5 \%$ for judging the significance of the coefficients and testing the null hypothesis behind the tests for Granger or quantile causality.

To control for the possible impact of the common dependence on the market, we test for Granger causality as well as for the various forms of quantile causality, also including the supercomposite US market index within the corresponding models 3

In the remainder of this paper, we identify the various networks that we estimate by means of acronyms. For networks estimated using quantile regression approaches (the baseline case, the quantile-on-quantile, and the non-parametric test), the acronym consists of four or five characters. The first and second identify the method: QR for baseline quantile regression; Qo for quantile-on-quantile; QN for non-parametric quantile. The third and fourth characters identify the quantile: for instance, QR10 refers to the network estimated by baseline quantile regression at the $10 \%$ quantile. For the quantile-on-quantile cases, the reported quantile is used for both the dependent and the explanatory variables. For standard Granger causality networks we use GR. Then we add a final letter $\mathrm{F}$ to the acronym if we allow for the presence of a common factor in our estimation of the causality networks.

Figures 24 4 graphically represent selected networks. Appendix C includes graphs for all the estimated causality networks.

Figure 2 includes four different networks among the largest US banks: the Granger causality network and three different quantile causality networks estimated by focusing on the $10 \%$ quantile. We indicate the baseline quantile network, the quantile-on-quantile network and the non-parametric quantile causality network. All the networks refer to the financial crisis period (2006 to 2008), and are estimated without any common factors. Figures 3 and 4 show similar networks estimated for the 25 largest US insurance companies and the US industry portfolios, respectively. We stress that we report results for the $10 \%$ quantile because we wish to shed

\footnotetext{
${ }^{3}$ We obtain the market return from the Fama/French 3 Factors dataset provided by Kenneth R. French and available at http://mba.tuck.dartmouth.edu/pages/faculty/ken.french/.
} 


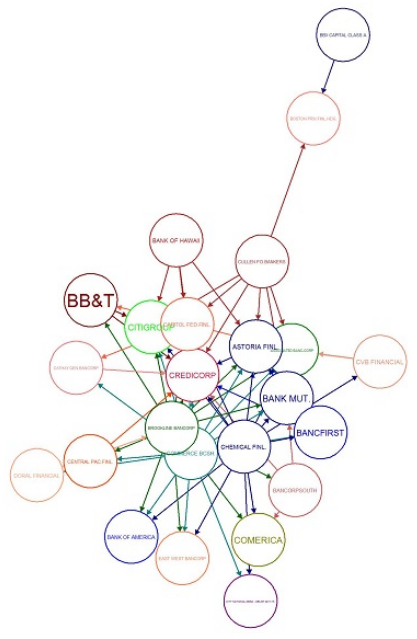

(a) Granger

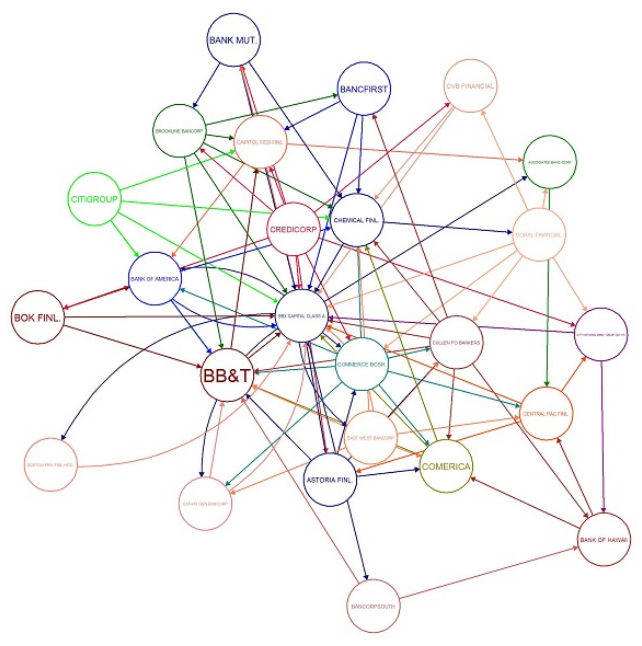

(c) Quantile on Quantile

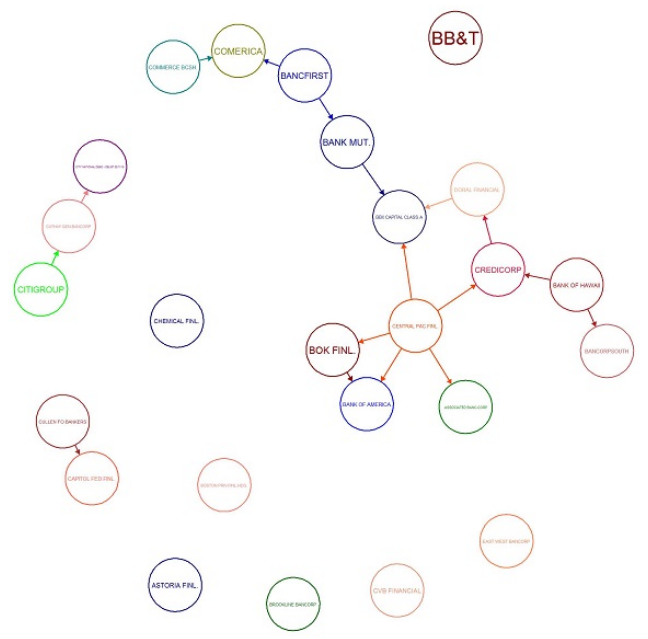

(b) Baseline Quantile

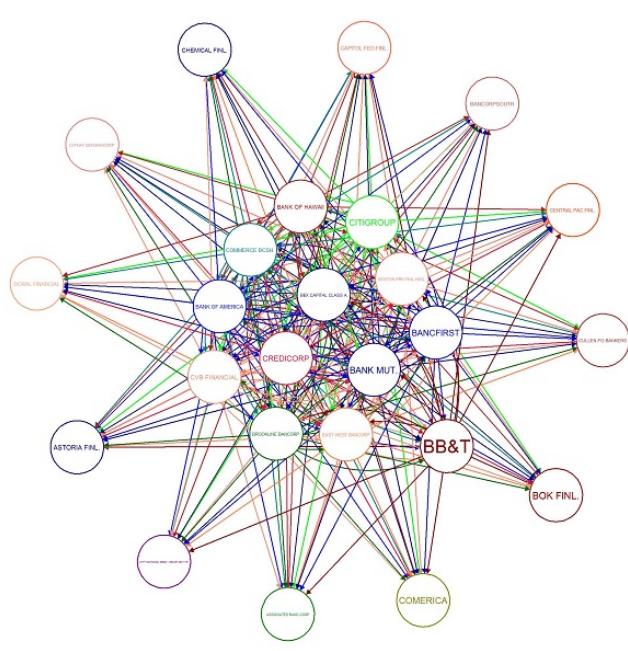

(d) Non-parametric Quantile

Figure 2: The figure visualizes 4 different networks for the period 2006-2008 relative to the first 25 banks ordered for market capitalization and listed in Appendix A. Panel a) reports the network extracted by the standard Granger causality approach of (Billio et al., 2012). Panel b) reports the Network extracted from a baseline quantile regression methodology, at the $10 \%$ quantile. Panel c) plots the network extracted from a quantile-on-quantile methodology at the $10 \%$ quantile. Panel d) graphs the network estimated by a non-parametric quantile causality methodology at the $10 \%$ quantile. All the estimates exclude the presence of common factors. We use the Fruchterman \& Reingold algorithm for network visualization. 


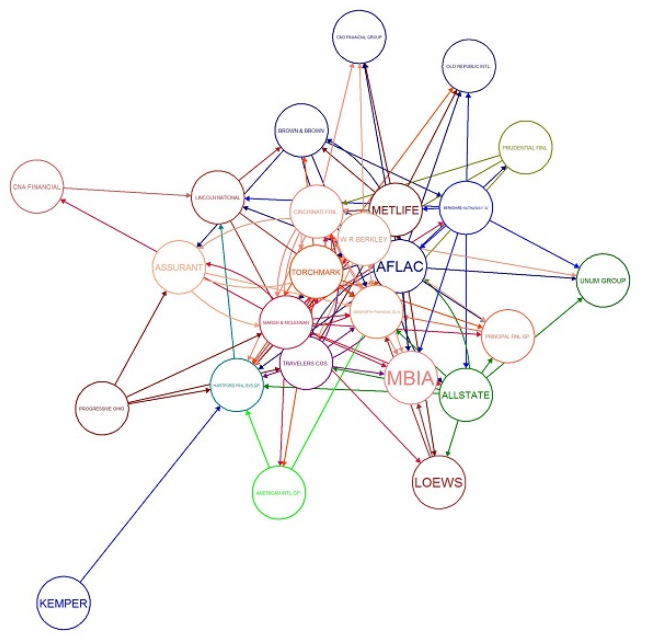

(a) Granger

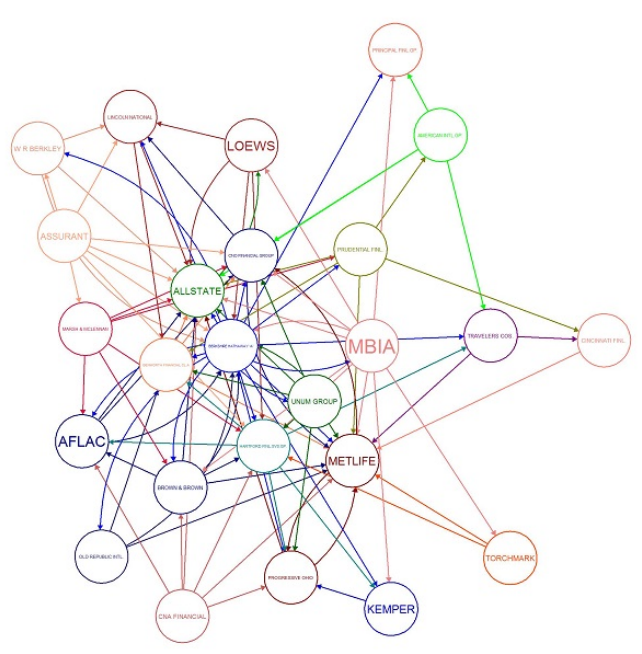

(c) Quantile on Quantile

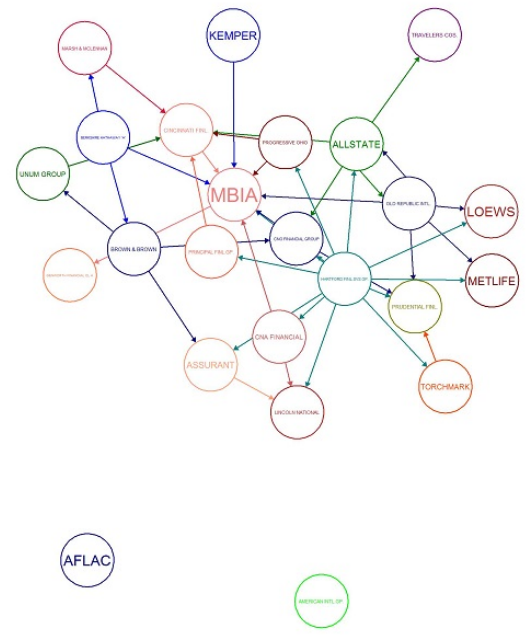

(b) Baseline Quantile

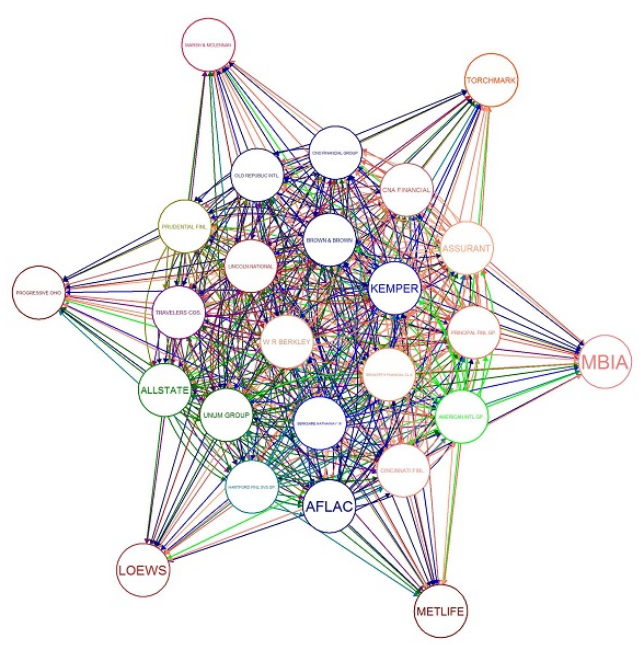

(d) Non-parametric Quantile

Figure 3: The figure visualizes 4 different networks for the period 2006-2008 relative to the first 25 insurances ordered for market capitalization and listed in Appendix A. Panel a) reports the network extracted by the standard Granger causality approach of (Billio et al., 2012). Panel b) reports the Network extracted from a baseline quantile regression methodology, at the $10 \%$ quantile. Panel c) plots the network extracted from a quantile-on-quantile methodology at the $10 \%$ quantile. Panel d) graphs the network estimated by a non-parametric quantile causality methodology at the $10 \%$ quantile. All the estimates exclude the presence of common factors. We use the Fruchterman \& Reingold algorithm for network visualization. 


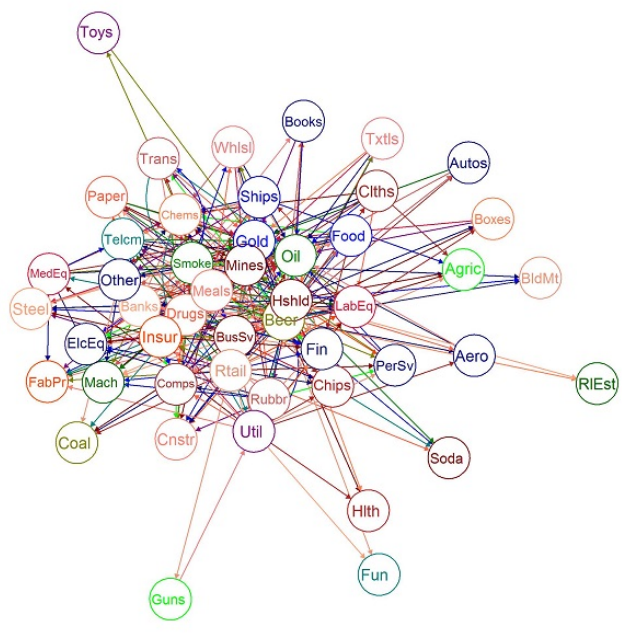

(a) Granger

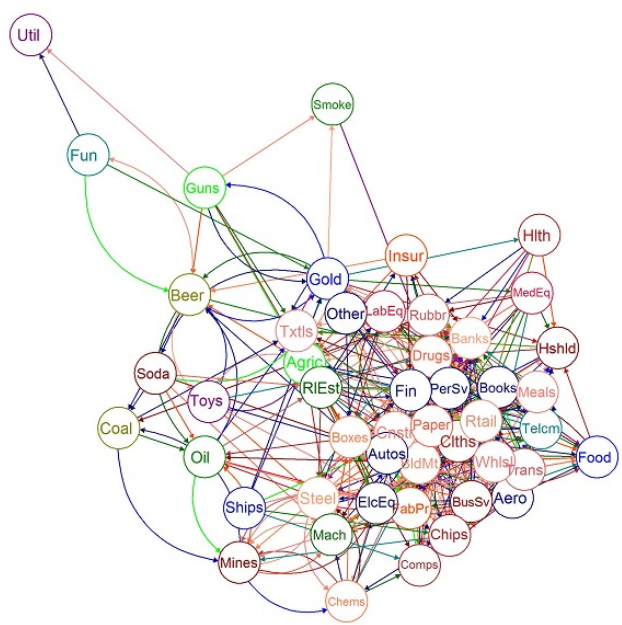

(c) Quantile on Quantile

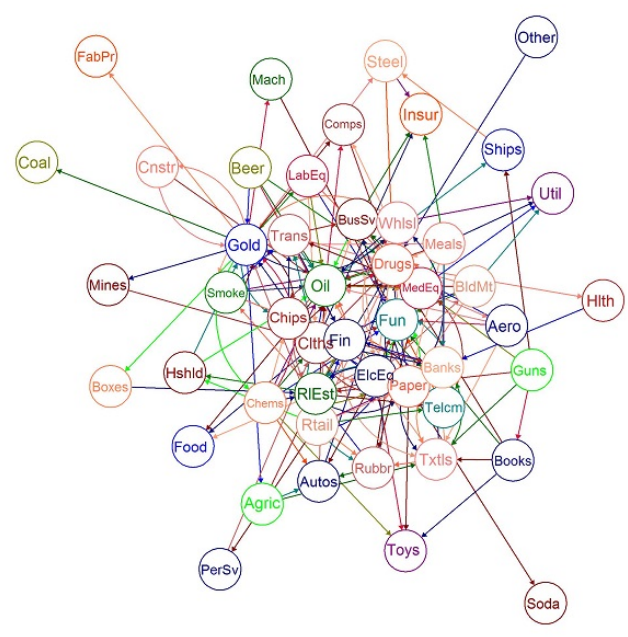

(b) Baseline Quantile

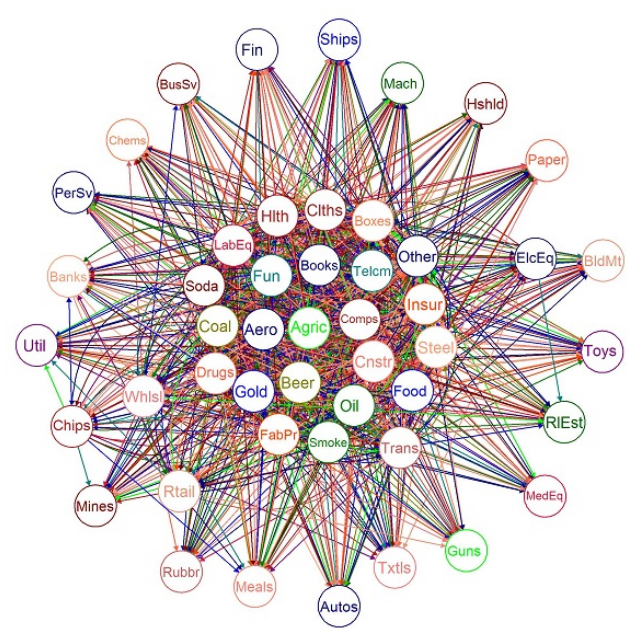

(d) Non-parametric Quantile

Figure 4: The figure visualizes 4 different networks for the period 2006-2008 relative to the 48 Industry portfolios obtained from the Kenneth French website. Panel a) reports the network extracted by the standard Granger causality approach of (Billio et al. 2012$)$. Panel b) reports the Network extracted from a baseline quantile regression methodology, at the $10 \%$ quantile. Panel c) plots the network extracted from a quantile-on-quantile methodology at the $10 \%$ quantile. Panel d) graphs the network estimated by a non-parametric quantile causality methodology at the $10 \%$ quantile. All the estimates exclude the presence of common factors. We use the Fruchterman \& Reingold algorithm for network visualization. 


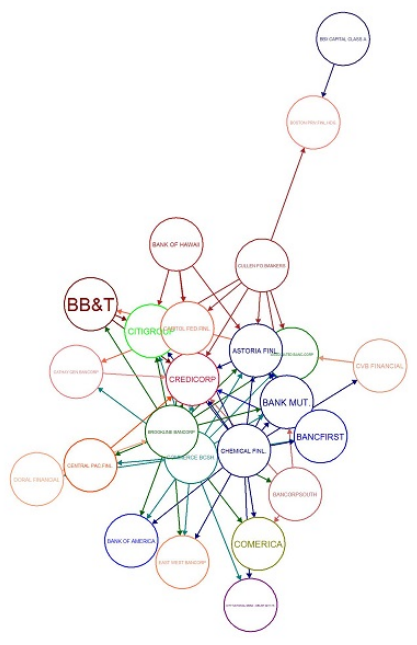

(a) Granger 2006-2008

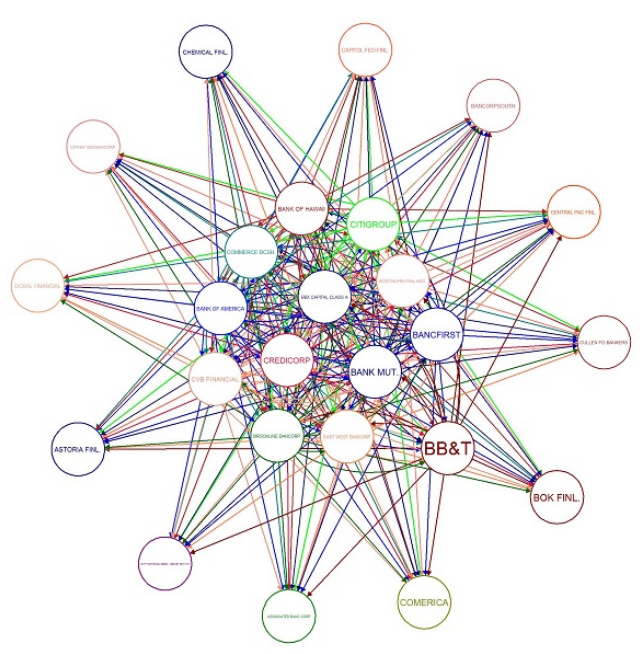

(c) Non-parametric quantile 2006-2008

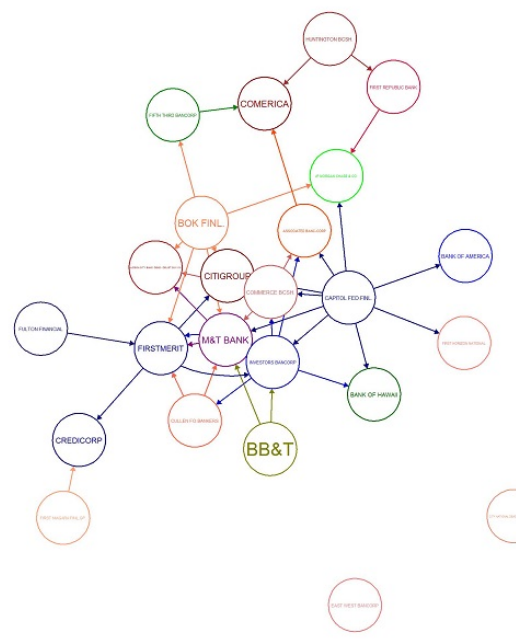

(b) Granger 2011-2015

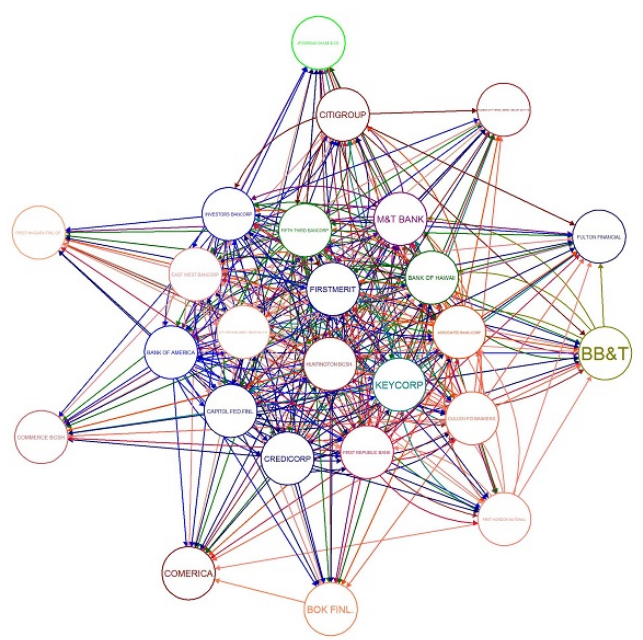

(d) Non-parametric quantile 2011-2015

Figure 5: The figure displays the network of the 25 banks ordered for market capitalization (see Appendix A by contrasting methods and samples. Panels a) and b) report the networks extracted by using the standard Granger causality methodology for the $2006-2008$ and $2011-2015$ samples, respectively. Panels c) and d) report the networks extracted by using the the nonparametric quantile causality test at the 10\% quantile for samples 2006-2008 and $2011-2015$, respectively. All the estimates exclude the presence of common factors. We use the Fruchterman \& Reingold algorithm for network visualization. 
some light on the possible differences between Granger causality (which focuses on the mean) and quantile causality (which places more emphasis on the tails). Networks that are based on quantile causality in the mean and also pay attention to the upper tail are available in Appendix C

Figure 2 shows that the quantile causality network extracted using the baseline quantile regression method has a majority of isolated nodes, whereas the network extracted with a non-parametric quantile causality test suggests the presence of a much greater density. The comparison between the networks of the banks and those of the insurance companies and industry portfolios shows that the former are less connected whatever methods is used. The non-parametric quantile causality test also generates networks characterized by a similar topology for all institutions and portfolios; in particular, it is easy to distinguish between a kernel and a periphery

Figure 5 shows the dataset of 25 banks over time, comparing the networks estimated for 2006 - 2008 with those obtained for 2011 - 2015, using two specific causality network estimation methods: Granger causality and non-parametric quantile causality (10\% quantile). We can see some changes in the Granger network structure when moving from the earlier to the later period. These changes are less clear when we consider non-parametric quantile causality, in which case the network still presents a kernel-periphery structure.

These preliminary graphical analyses suggest that there are potentially relevant differences between the networks estimated using Granger causality as opposed to quantile-based causality approaches. The latter have a more clear focus on risk than the former. Before moving on to the combination of the estimated networks, we run a comparison of the estimated networks using summary measures.

We consider four different indicators: Density, Assortativity, and two versions of Eigenvector centrality. Density monitors the number of connections between nodes. A higher density is the sign of a large number of connections between nodes, which are consequently more closely related to one another. Assortativity captures the nodes' tendency to connect with other nodes having similar properties; it can take values in the range of $(-1,1)$. For values close to 1 , the network has an assortative behavior, with nodes being connected to other nodes that have similar degrees; for values nearing zero, the network becomes similar to a random graph. A disassortative behavior, when high-degree nodes point to low-degree nodes, corresponds to assortativity values close to -15 Eigenvector centrality monitors the relevance of each node as a function of the relevance of neighboring nodes. We consider two versions: one based on the non-normalized adjacency matrix, and one based on the row-normalized adjacency matrix. We also normalize the eigenvector centrality of each node to the maximum score obtained by the most connected node. The eigenvector centrality value thus ranges from zero to one, and can easily be compared across networks. In the summary tables, we focus on the average eigenvector centrality across nodes. Changes in the eigenvector centrality are a sign of changes in the network structure. Appendix D provides additional details on the network measures considered. Tables from 1 to

\footnotetext{
${ }^{4}$ We use the Fruchterman \& Reingold algorithm for network visualization (Fruchterman and Reingold, 1991).

${ }^{5}$ The few blank spaces appearing in the following tables of network summary measures correspond to indeterminate forms. The indeterminate forms of the assortativity and eigenvector centrality measures are discussed in the Appendix $\mathrm{D}$.
} 
Table 1: This table reports summary measures for the networks estimated from competing causality methodologies over the 25 Banks listed in Appendix A. QB stands for baseline quantile, Qo for quantile-on-quantile, QN for non-parametric quantile causality, GR for Granger causality. Numbers identify the reference quantile for quantile-based causality networks, 10 for $10 \%, 50$ for the median and 90 for $90 \%$. An F at the end of the acronyms specifies that the underlying models included the market index as a common factor.

\begin{tabular}{lcccccc}
\hline & \multicolumn{2}{c}{ Density } & \multicolumn{2}{c}{ Assortativity } & \multicolumn{2}{c}{$\begin{array}{c}\text { Eigenvector } \\
\text { Centrality }\end{array}$} \\
\hline \hline GR & $\mathbf{0 6 - 0 8}$ & $\mathbf{1 1 - 1 5}$ & $\mathbf{0 6 - 0 8}$ & $\mathbf{1 1 - 1 5}$ & $\mathbf{0 6 - 0 8}$ & $\mathbf{1 1 - 1 5}$ \\
\hline QB10 & 0.12 & 0.07 & -0.37 & -0.22 & 0.12 & 0.26 \\
QB50 & 0.03 & 0.04 & 0.05 & -0.13 & & \\
QB90 & 0.05 & 0.07 & 0.03 & -0.06 & 0.16 & \\
\hline Qo10 & 0.12 & 0.13 & -0.44 & -0.22 & 0.25 & 0.11 \\
Q050 & 0.16 & 0.36 & -0.37 & -0.28 & 0.23 & 0.45 \\
Q090 & 0.06 & 0.03 & -0.43 & 0.00 & 0.34 & \\
\hline QN10 & 0.17 & 0.35 & -0.53 & -0.07 & 0.25 & 0.44 \\
QN50 & 0.51 & 0.63 & -0.11 & -0.20 & 0.93 & 0.83 \\
QN90 & 0.29 & 0.33 & -0.30 & -0.28 & 0.75 & 0.72 \\
\hline \hline GRF & 0.57 & 0.52 & -0.15 & -0.13 & 0.90 & 0.83 \\
\hline QB10F & 0.07 & 0.07 & -0.01 & -0.24 & 0.22 & 0.31 \\
QB50F & 0.04 & 0.04 & -0.26 & 0.13 & 0.08 & \\
QB90F & 0.04 & 0.09 & -0.23 & -0.12 & 0.14 & 0.29 \\
\hline Qo10F & 0.10 & 0.09 & -0.37 & -0.34 & 0.21 & 0.26 \\
Qo50F & 0.22 & 0.50 & -0.29 & -0.24 & 0.24 & 0.50 \\
Q090F & 0.06 & 0.03 & -0.07 & -0.25 & 0.18 & \\
\hline QN10F & 0.10 & 0.51 & -0.50 & -0.20 & 0.18 & 0.57 \\
QN50F & 0.52 & 0.70 & -0.14 & -0.23 & 0.88 & 0.84 \\
QN90F & 0.34 & 0.38 & -0.22 & -0.21 & 0.80 & 0.81 \\
\hline \hline Average & 0.64 & 0.64 & -0.09 & -0.14 & 0.96 & 0.89 \\
Standard Deviation & $\mathbf{0 . 2 0}$ & $\mathbf{0 . 2 4}$ & $\mathbf{0 . 1 7}$ & $\mathbf{0 . 1 1}$ & $\mathbf{0 . 3 3}$ & $\mathbf{0 . 2 6}$ \\
\hline
\end{tabular}

3 contain the summary measures.

Table 1 contains the network measures for the banks dataset. We can see that, on average, the density is slightly smaller during the crisis, while the disassortative behavior (seen in both periods) is higher. Granger's causality networks (with/without the common factor) have small densities that remain almost constant in the two samples, and a disassortative behavior, and the average eigenvector centrality increases in the most recent period. The values for the quantilebased networks are quite different, as the previous graphical analysis suggests. In particular, the networks derived by means of the non-parametric quantile causality test have the highest density, combined with a disassortative pattern. These two elements could explain the kernel-periphery structure exhibited in the Figure 2 .

A high density combined with a disassortative pattern is also associated with very high eigenvector centrality averages, which indicate that the nodes' relevance is quite evenly distributed, a somewhat expected result given that we focus on the largest banks. Notably, the picture is 
quite different in the Granger causality networks, which are characterized by a lower average eigenvector centrality. From a systemic risk perspective, this finding suggests that, while the Granger causality analysis provides evidence of few relevant nodes that are more central to the network structure, a non-parametric quantile causality test leads to the construction of a denser network where the risk is more evenly distributed across nodes, and many nodes (more than those emerging from Granger causality) are systemically relevant. As the graphs suggest, the non-parametric quantile causality networks also differ from those based on other quantile-based approaches. In particular, the baseline quantile case provides summary measures more closely resembling those of Granger causality, while the quantile-on-quantile cases have density measures higher than with Granger causality but lower than with non-parametric quantile causality, and they show the most disassortative behaviors. We note some interesting differences across quantiles, with higher densities on the extreme quantiles (10\% and 90\%) than on the median case, which comes the closest to the Granger causality. Notably, this would mean that the network structure changes if we move away from the mean (median), an important aspect to bear in mind if our purpose is to analyze the spread of risk in times of market turmoil. Finally, the introduction of a common market factor seems to have a limited impact.

Table 2 indicates that the insurance companies dataset behaves in much the same way as the banks dataset (thus confirming our previous comments), with a marked change in the network structure when moving from Granger causality to quantile causality. The most relevant difference concerns network density, which is much higher in the tails than in the mean (which coincides with Granger causality). We also find the highest average eigenvector centrality coinciding with non-parametric quantile causality.

Table 3 shows the summary measures for the causality network estimated from the 48 industry portfolios. Here again, we find relevant differences between the summary measures for the Granger and quantile causality networks. This holds particularly for network density and average eigenvector centrality. We note, however, that the structure of the non-parametric quantile causality networks differs considerably from the other quantile causality networks in all three datasets.

The graphical analysis and summary measures confirm that, changing our approach to estimating a causality network, coincides with important changes in the network structure. To examine the possible relevance of either the Granger causality network or the quantile causality networks, we therefore proceed, in the following section, with the estimation of a composite network.

\subsection{The composite causality network}

Starting from the availability of causality networks estimated using four different methods (Granger, Quantile Regression, Quantile-on-Quantile and Non-parametric Quantile causality), and over different quantiles (10\%, the median and $90 \%)$ for three of them, we now proceed to estimate composite networks.

We estimate the model in equation (5), accounting for the presence of common factors, for which we follow the usual practice and introduce the market factor, the size factor, the book-tomarket factor, and the momentum factor, following Fama and French (1993), Fama and French 
Table 2: This table reports summary measures for the networks estimated from competing causality methodologies over the 25 Insurance companies listed in Appendix A. QB stands for baseline quantile, Qo for quantile-on-quantile, QN for non-parametric quantile causality, GR for Granger causality. Numbers identify the reference quantile for quantile-based causality networks, 10 for $10 \%, 50$ for the median and 90 for $90 \%$. An $\mathrm{F}$ at the end of the acronyms specifies that the underlying models included the market index as a common factor.

\begin{tabular}{|c|c|c|c|c|c|c|}
\hline & \multicolumn{2}{|c|}{ Density } & \multicolumn{2}{|c|}{ Assortativity } & \multicolumn{2}{|c|}{$\begin{array}{c}\text { Eigenvector } \\
\text { Centrality }\end{array}$} \\
\hline & $06-08$ & 11-15 & 06-08 & 11-15 & 06-08 & 11-15 \\
\hline GR & 0.20 & 0.03 & -0.29 & -0.37 & 0.34 & \\
\hline QB10 & 0.07 & 0.06 & -0.36 & 0.15 & 0.15 & 0.22 \\
\hline QB50 & 0.07 & 0.14 & -0.17 & -0.15 & 0.32 & 0.36 \\
\hline QB90 & 0.04 & 0.07 & -0.44 & -0.03 & & \\
\hline Qo10 & 0.17 & 0.25 & -0.31 & -0.29 & 0.29 & 0.33 \\
\hline Qo50 & 0.03 & 0.05 & 0.00 & -0.10 & & \\
\hline Qo90 & 0.22 & 0.21 & -0.42 & -0.08 & 0.38 & 0.30 \\
\hline QN10 & 0.76 & 0.56 & & -0.23 & 0.96 & 0.90 \\
\hline QN50 & 0.41 & 0.50 & -0.35 & -0.18 & 0.70 & 0.85 \\
\hline QN90 & 0.76 & 0.59 & -0.02 & -0.20 & 0.96 & 0.82 \\
\hline GRF & 0.15 & 0.04 & -0.31 & -0.40 & 0.26 & \\
\hline QB10F & 0.04 & 0.08 & -0.49 & -0.12 & & 0.22 \\
\hline QB50F & 0.05 & 0.12 & -0.06 & -0.05 & & 0.27 \\
\hline QB90F & 0.04 & 0.10 & -0.44 & -0.08 & & 0.37 \\
\hline Qo10F & 0.08 & 0.43 & -0.06 & -0.28 & 0.18 & 0.44 \\
\hline Qo50F & 0.04 & 0.05 & 0.13 & 0.21 & & 0.21 \\
\hline Qo90F & 0.10 & 0.45 & -0.43 & -0.31 & 0.24 & 0.46 \\
\hline QN10F & 0.76 & 0.66 & & -0.11 & 0.96 & 0.93 \\
\hline QN50F & 0.54 & 0.55 & -0.13 & -0.11 & 0.92 & 0.93 \\
\hline QN90F & 0.76 & 0.62 & & -0.18 & 0.96 & 0.86 \\
\hline Average & 0.26 & 0.28 & -0.24 & -0.14 & 0.54 & 0.53 \\
\hline Standard Deviation & 0.29 & 0.23 & 0.19 & 0.15 & 0.34 & 0.29 \\
\hline
\end{tabular}


Table 3: This table reports summary measures for the networks estimated from competing causality methodologies over the 48 Industry portfolios recovered from the Kenneth French website. QB stands for baseline quantile, Qo for quantile-on-quantile, QN for non-parametric quantile causality, GR for Granger causality. Numbers identify the reference quantile for quantilebased causality networks, 10 for 10\%, 50 for the median and 90 for $90 \%$. An F at the end of the acronyms specifies that the underlying models included the market index as a common factor.

\begin{tabular}{lcccccc}
\hline & \multicolumn{2}{c}{ Density } & \multicolumn{2}{c}{ Assortativity } & \multicolumn{2}{c}{$\begin{array}{c}\text { Eigenvector } \\
\text { Centrality }\end{array}$} \\
\hline & $\mathbf{0 6 - 0 8}$ & $\mathbf{1 1 - 1 5}$ & $\mathbf{0 6 - 0 8}$ & $\mathbf{1 1 - 1 5}$ & $\mathbf{0 6 - 0 8}$ & $\mathbf{1 1 - 1 5}$ \\
\hline GR & 0.14 & 0.02 & -0.49 & -0.74 & 0.15 & 0.36 \\
\hline QB10 & 0.09 & 0.06 & -0.39 & -0.20 & 0.24 & 0.24 \\
QB50 & 0.16 & 0.06 & -0.27 & -0.13 & 0.21 & 0.27 \\
QB90 & 0.14 & 0.07 & -0.24 & -0.37 & 0.29 & 0.29 \\
\hline Qo10 & 0.20 & 0.52 & -0.06 & -0.10 & 0.29 & 0.60 \\
Q050 & 0.08 & 0.06 & 0.09 & -0.13 & 0.25 & 0.13 \\
Q090 & 0.14 & 0.48 & -0.29 & -0.15 & 0.26 & 0.56 \\
\hline QN10 & 0.52 & 0.42 & -0.25 & -0.14 & 0.88 & 0.90 \\
QN50 & 0.32 & 0.28 & -0.22 & -0.23 & 0.82 & 0.83 \\
QN90 & 0.57 & 0.48 & -0.14 & -0.18 & 0.93 & 0.90 \\
\hline \hline GRF & 0.15 & 0.02 & -0.33 & -0.71 & 0.18 & 0.16 \\
\hline QB10F & 0.14 & 0.04 & -0.11 & -0.11 & 0.17 & 0.18 \\
QB50F & 0.15 & 0.06 & -0.17 & -0.21 & 0.20 & 0.16 \\
QB90F & 0.09 & 0.08 & -0.12 & 0.02 & 0.17 & 0.18 \\
\hline Qo10F & 0.39 & 0.79 & -0.28 & -0.12 & 0.43 & 0.79 \\
Qo50F & 0.09 & 0.07 & -0.19 & -0.10 & 0.13 & 0.10 \\
Q090F & 0.35 & 0.82 & -0.27 & -0.09 & 0.39 & 0.82 \\
\hline QN10F & 0.59 & 0.47 & -0.12 & -0.18 & 0.95 & 0.88 \\
QN50F & 0.33 & 0.33 & -0.18 & -0.22 & 0.85 & 0.81 \\
QN90F & 0.59 & 0.51 & -0.15 & -0.11 & 0.93 & 0.93 \\
\hline \hline Average & $\mathbf{0 . 2 6}$ & $\mathbf{0 . 2 8}$ & $\mathbf{- 0 . 2 1}$ & $\mathbf{- 0 . 2 1}$ & $\mathbf{0 . 4 4}$ & $\mathbf{0 . 5 1}$ \\
Standard Deviation & $\mathbf{0 . 1 8}$ & $\mathbf{0 . 2 6}$ & $\mathbf{0 . 1 2}$ & $\mathbf{0 . 1 9}$ & $\mathbf{0 . 3 2}$ & $\mathbf{0 . 3 2}$ \\
\hline
\end{tabular}


(1995), and Carhart (1997). We have no information a priori to suggest a possible preference for particular network, so we estimate the composite network starting from four different layers, where the quantile-based networks are associated with the same reference quantile. We provide a sensitivity analysis on the effect of excluding a single network in the robustness checks section.

Then we estimate the linear factor model, focusing on weekly returns. While it is more common to consider the monthly frequency when estimating factor models, this would leave us with only 36 observations in the first sample, while we have 60 monthly returns in the second. Hence our decision to focus on the weekly frequency, which enables us to increase the number of returns for the factor model (augmented with network dependence) to 108 in the first sample and 316 in the second. This choice has advantages in terms of model estimation and parameter inference. We do not consider daily data as they would be bound to require the introduction of heteroskedastic dynamics in the variance of the residuals, adding to the complexity of the estimation due to the well-known curse of dimensionality. Estimates of the network combination on monthly returns are nonetheless provided in the robustness checks sections.

The estimates of the linear factor model with network dependence generate different outputs. First, there are the model parameters: the weight of each network, as measured by the $\delta_{i}$ parameters, and the impact of the composite network on the asset returns, the parameters included in $\mathcal{R}$. Second, comes the composite network, obtained by combining the primitive networks weighted with the $\delta_{i}$ coefficients. Finally, there are the model residuals, which contain information useful for assessing the advantages of moving to a composite network as opposed to the benchmark cases of no network dependence (where the contemporaneous link matrix $A$ is an identity), or a network dependence captured by Granger causality. The latter is a valuable benchmark as we would like to underscore the potential improvement associated with measuring asset links going beyond the mean (i.e. on the quantiles). To compare models, we use the residuals average correlation: if we see improvements, we expect to have residuals characterized by a smaller correlation level, i.e. more whitened residuals.

Table 4 shows the parameters estimated for the combined network in the three datasets and over sample periods. Bearing in mind that the sum of these parameters is 1 and they are all positive, a higher value of a parameter indicates a greater relevance of the associated network. We also assess the significance of the estimated parameters. Within the banks dataset, we note that the non-parametric quantile causality network is the most relevant during the financial crisis period and across all quantiles. Notably, the estimated $\delta$ parameter for this network ranges between 0.736 and 0.883 , and it is always statistically significant when the estimation of the networks takes the presence of a common market factor into account.

We stress that, from a systemic risk perspective, this result suggests that the risk is more widespread across banks, as the non-parametric quantile network is much denser than the other causality networks. This greater relevance of the non-parametric quantile causality network also emerges for the median and for the lower and upper tails. In all cases, the Granger causality network has a very small weight (which is non-significant in five cases out of six). Switching to the more recent period considered, both the non-parametric quantile causality network and the quantile-on-quantile causality network are relevant, with and without the introduction of a common market factor. Here again, although it is more statistically significant, the Granger 
Table 4: The table reports the $\delta$ of model (5) that represent the weights for networks combination. The top panel focused on the banks dataset, the middle panel on the insurance companies dataset and the bottom panel on the industry portfolios dataset. The first column identifies the quantiles used to estimate the quantile-based network, and the second column indicates if a common factor was used $(\mathrm{Y})$ or not used $(\mathrm{N})$ in the estimation of the causality networks. Columns 3 to 6 refer to the crisis sample while columns 7 to 10 to the most recent sample. The second row identifies the four different networks which are optimally combined: baseline quantile causality - QB; quantile-on-quantile causality Qo; non-parametric quantile causality QN; Granger causality. Parameters are, by construction, positive and sum up to one (within each row and within each period). A star identifies parameters significant at the $5 \%$ confidence level.

\begin{tabular}{|c|c|c|c|c|c|c|c|c|c|}
\hline \multirow[b]{2}{*}{ Quantile } & \multirow[b]{2}{*}{ Factor } & \multicolumn{4}{|c|}{ 2006-2008 } & \multicolumn{4}{|c|}{ 2010-2015 } \\
\hline & & QB & Qo & QN & GR & QB & Qo & QN & GR \\
\hline & & \multicolumn{8}{|c|}{25 Banks } \\
\hline $10 \%$ & $\mathrm{~N}$ & 0.061 & 0.055 & 0.875 & 0.010 & 0.000 & $0.630^{*}$ & $0.354^{*}$ & $0.017^{*}$ \\
\hline $50 \%$ & $\mathrm{~N}$ & 0.146 & 0.108 & $0.736^{*}$ & 0.010 & $0.091^{*}$ & $0.065^{*}$ & $0.773^{*}$ & 0.071 \\
\hline $90 \%$ & $\mathrm{~N}$ & 0.005 & 0.008 & 0.883 & 0.104 & 0.000 & $0.706^{*}$ & 0.243 & $0.051^{*}$ \\
\hline $10 \%$ & $\mathrm{Y}$ & 0.022 & 0.073 & $0.827^{*}$ & 0.077 & 0.000 & $0.538^{*}$ & $0.428^{*}$ & $0.034^{*}$ \\
\hline $50 \%$ & $\mathrm{Y}$ & 0.052 & 0.057 & $0.842^{*}$ & 0.050 & 0.107 & $0.072^{*}$ & $0.649^{*}$ & $0.173^{*}$ \\
\hline \multirow[t]{2}{*}{$90 \%$} & $\mathrm{Y}$ & 0.134 & $0.000^{*}$ & $0.762^{*}$ & $0.104^{*}$ & 0.000 & $0.876^{*}$ & 0.121 & $0.003^{*}$ \\
\hline & & \multicolumn{8}{|c|}{25 Insurance Companies } \\
\hline $10 \%$ & $\mathrm{~N}$ & 0.000 & $0.022^{*}$ & $0.951^{*}$ & 0.027 & 0.058 & 0.831 & 0.111 & 0.000 \\
\hline $50 \%$ & $\mathrm{~N}$ & $0.865^{*}$ & 0.000 & $0.032^{*}$ & 0.103 & 0.059 & 0.045 & $0.886^{*}$ & 0.010 \\
\hline $90 \%$ & $\mathrm{~N}$ & 0.000 & 0.028 & $0.972^{*}$ & 0.000 & 0.000 & $0.364^{*}$ & $0.436^{*}$ & 0.201 \\
\hline $10 \%$ & $\mathrm{Y}$ & 0.001 & 0.006 & $0.948^{*}$ & 0.045 & 0.000 & 0.488 & 0.512 & 0.000 \\
\hline $50 \%$ & $\mathrm{Y}$ & $0.731^{*}$ & 0.054 & $0.188^{*}$ & $0.027^{*}$ & 0.051 & 0.040 & $0.837^{*}$ & 0.072 \\
\hline \multirow[t]{2}{*}{$90 \%$} & $\mathrm{Y}$ & 0.000 & 0.046 & $0.954^{*}$ & 0.000 & 0.000 & 0.554 & 0.446 & 0.000 \\
\hline & & \multicolumn{8}{|c|}{48 Industry Portfolios } \\
\hline $10 \%$ & $\mathrm{~N}$ & 0.000 & $1.000^{*}$ & 0.000 & 0.000 & 0.032 & $0.968^{*}$ & 0.000 & 0.000 \\
\hline $50 \%$ & $\mathrm{~N}$ & 0.212 & 0.000 & 0.478 & 0.310 & 0.021 & $0.031^{*}$ & $0.848^{*}$ & 0.100 \\
\hline $90 \%$ & $\mathrm{~N}$ & 0.108 & $0.892^{*}$ & 0.000 & 0.000 & 0.000 & $1.000^{*}$ & 0.000 & 0.000 \\
\hline $10 \%$ & $\mathrm{Y}$ & 0.178 & 0.471 & 0.000 & 0.351 & 0.036 & $0.695^{*}$ & 0.228 & 0.041 \\
\hline $50 \%$ & $\mathrm{Y}$ & 0.123 & 0.000 & 0.000 & $0.877^{*}$ & 0.092 & $0.007^{*}$ & $0.836^{*}$ & 0.064 \\
\hline $90 \%$ & $\mathrm{Y}$ & 0.000 & 0.810 & 0.000 & 0.190 & 0.107 & 0.808 & 0.030 & 0.055 \\
\hline
\end{tabular}

causality network has smaller weights than the quantile-based causality networks. Overall, in both samples, the baseline quantile regression network has the least relevant role. Finally, in the second sample, we see a clear change in the combined network parameters when moving from median networks to the use of networks estimated on either the lower or upper tails. In particular, the impact of the quantile-on-quantile networks increases, thus supporting the importance of looking at quantile causality too when accounting for interdependence across assets in a linear factor model, and showing that a double conditioning (on both the dependent and the explanatory variables) is important when estimating causality at quantile level.

The insurance companies dataset produces somewhat similar results. In both periods, Granger causality networks are the least relevant, being associated with small coefficients (and 
Table 5: The table reports summary measures of the composite networks estimated from model (5) over the different datasets, sample periods, and reference quantiles. In all cases the composite networks is formed by the combination of four networks: the baseline quantile causality network, the quantile-on-quantile causality network, the non-parametric quantile causality network, the Granger causality network. The top panel focused on the banks dataset, the middle panel on the insurance companies dataset and the bottom panel on the industry portfolios dataset. The first column identifies the quantiles used to estimate the quantile-based networks, and the second column indicates if a common factor was used $(\mathrm{Y})$ or not used $(\mathrm{N})$ in the estimation of the causality networks.

\begin{tabular}{|c|c|c|c|c|c|c|c|c|c|}
\hline \multicolumn{2}{|c|}{ Measures } & \multicolumn{2}{|c|}{ Density } & \multicolumn{2}{|c|}{ Assortativity } & \multicolumn{2}{|c|}{$\begin{array}{c}\text { Eigenvector } \\
\text { Centrality }\end{array}$} & \multicolumn{2}{|c|}{$\begin{array}{c}\text { Eigenvector } \\
\text { Centrality } \\
\text { Adj Weighted }\end{array}$} \\
\hline Quantile & Factor & 06-08 & 11-15 & 06-08 & 11-15 & 06-08 & 11-15 & $06-08$ & $11-15$ \\
\hline & & \multicolumn{8}{|c|}{25 Banks } \\
\hline $10 \%$ & $\mathrm{~N}$ & 0.63 & 0.79 & -0.30 & -0.23 & 0.70 & 0.83 & 0.35 & 0.47 \\
\hline $50 \%$ & $\mathrm{~N}$ & 0.45 & 0.43 & -0.28 & -0.28 & 0.62 & 0.61 & 0.24 & 0.43 \\
\hline $90 \%$ & $\mathrm{~N}$ & 0.69 & 0.76 & -0.32 & -0.25 & 0.70 & 0.77 & 0.32 & 0.57 \\
\hline $10 \%$ & $\mathrm{Y}$ & 0.65 & 0.87 & -0.36 & -0.25 & 0.67 & 0.87 & 0.39 & 0.64 \\
\hline $50 \%$ & $\mathrm{Y}$ & 0.45 & 0.48 & -0.31 & -0.36 & 0.65 & 0.69 & 0.31 & 0.41 \\
\hline \multirow[t]{2}{*}{$90 \%$} & $\mathrm{Y}$ & 0.72 & 0.85 & -0.28 & -0.25 & 0.75 & 0.85 & 0.39 & 0.64 \\
\hline & & \multicolumn{8}{|c|}{25 Insurance Companies } \\
\hline $10 \%$ & $\mathrm{~N}$ & 0.88 & 0.72 & -0.17 & -0.29 & 0.88 & 0.74 & 0.66 & 0.45 \\
\hline $50 \%$ & $\mathrm{~N}$ & 0.59 & 0.61 & -0.22 & -0.21 & 0.72 & 0.85 & 0.37 & 0.60 \\
\hline $90 \%$ & $\mathrm{~N}$ & 0.87 & 0.72 & -0.15 & -0.24 & 0.91 & 0.77 & 0.66 & 0.50 \\
\hline $10 \%$ & $\mathrm{Y}$ & 0.82 & 0.82 & -0.22 & -0.30 & 0.85 & 0.82 & 0.55 & 0.59 \\
\hline $50 \%$ & $\mathrm{Y}$ & 0.66 & 0.64 & -0.25 & -0.23 & 0.79 & 0.84 & 0.55 & 0.51 \\
\hline \multirow[t]{2}{*}{$90 \%$} & $\mathrm{Y}$ & 0.83 & 0.83 & -0.22 & -0.26 & 0.83 & 0.83 & 0.60 & 0.59 \\
\hline & & \multicolumn{8}{|c|}{48 Industry Portfolios } \\
\hline $10 \%$ & $\mathrm{~N}$ & 0.71 & 0.74 & -0.22 & -0.26 & 0.77 & 0.78 & 0.46 & 0.62 \\
\hline $50 \%$ & $\mathrm{~N}$ & 0.50 & 0.37 & -0.30 & -0.29 & 0.58 & 0.51 & 0.29 & 0.19 \\
\hline $90 \%$ & $\mathrm{~N}$ & 0.71 & 0.74 & -0.21 & -0.24 & 0.76 & 0.76 & 0.38 & 0.62 \\
\hline $10 \%$ & $\mathrm{Y}$ & 0.83 & 0.89 & -0.24 & -0.24 & 0.83 & 0.90 & 0.51 & 0.69 \\
\hline $50 \%$ & $\mathrm{Y}$ & 0.53 & 0.42 & -0.35 & -0.37 & 0.56 & 0.55 & 0.24 & 0.23 \\
\hline $90 \%$ & $\mathrm{Y}$ & 0.80 & 0.92 & -0.29 & -0.15 & 0.80 & 0.92 & 0.47 & 0.70 \\
\hline
\end{tabular}

only one in twelve is statistically significant). In the first period considered, non-parametric quantile causality is the most relevant, although baseline quantile causality network receives a much larger weight in the median case (unlike the picture emerging from the banks dataset). In the second sample, both non-parametric quantile causality and quantile-on-quantile causality are relevant, with a change in the estimated parameters when moving from networks estimated on the median to networks estimated on the $10 \%$ or $90 \%$ quantiles.

Finally, for the industry portfolio dataset, the results are more heterogeneous in the first sample, while in the second they are consistent with the two previous cases. In the first period, the significance is limited in many cases, particularly for the quantile-based causality networks estimated at the median. In the second period, there is a marked difference between the quantile- 
based causality estimated at the median and those estimated on the tails. Be that as it may, Granger causality networks receive the smallest weight (and are never statistically significant).

Table 5 shows the summary measures for the combined networks. The various composite networks are similar in all the quantities we report (for a given sample period, and a given dataset). The heterogeneity identified is much smaller than was seen for the primitive networks. Figures 6 to 8 show the combined networks for the period $2006-2008$.

We link this finding to the use of a linear factor model augmented with network dependence. The contemporaneous relation across the modelled variables captures the correlation across these variables and goes beyond what we associate with common factors. It might be that the various composite networks capture the dependence across the returns (beyond common factors in a similar way. The differences we find depend partly on the heterogeneity across networks and partly on the different weights assigned to the primitive networks.

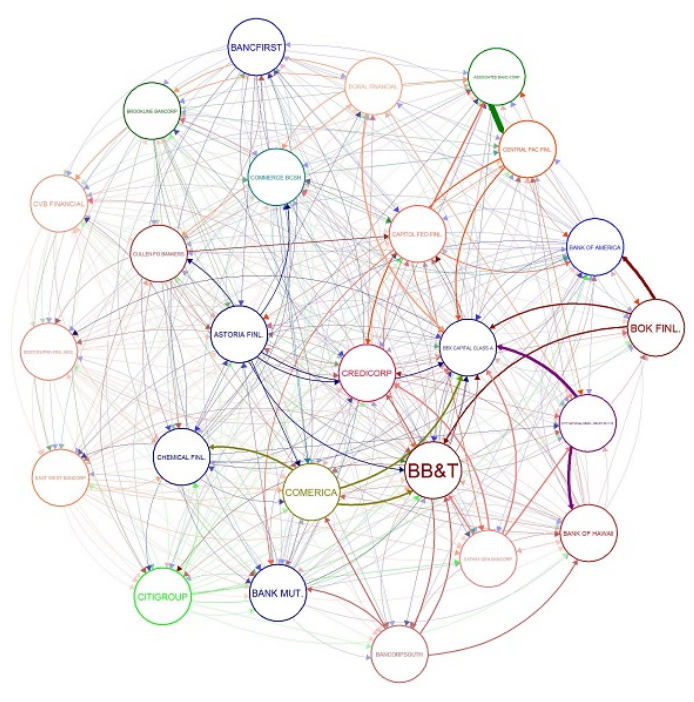

Figure 6: The figure visualizes the network for the Banks companies dataset. The network is extracted by combining causality network by using quantile regression (QB, Qo and QN) at the $10 \%$ quantile, and the standard granger causality method, during the period 2006-2008. In this case we do not allow the presence of the common factor for network estimations.

To shed further light on the differences between the composite networks and generate some evidence of the improvement gained by the linear factor model augmented with network combination, we provide descriptive analyses -in Tables 6 to 8 of the correlations between the model residuals (5). The tables include two benchmarks: a standard linear factor model, the (Carhart, 1997) 4-factors CAPM; and a linear factor augmented with network dependence where the latter is the Granger causality network (with or without the inclusion of a common factor in estimating causal relationships). For the datasets concerning the banks and insurance companies, the results are much the same. The residuals of the 4-factors CAPM have the largest median correlations, and a distribution of these correlations shifted to the right, with a clear prevalence of positive values. The introduction of network dependence, when measured by Granger causality, improves the model fit, but the predominance of positive values in the correlation remains (see the small fraction of correlations below -0.1), and the median correlation remains above 


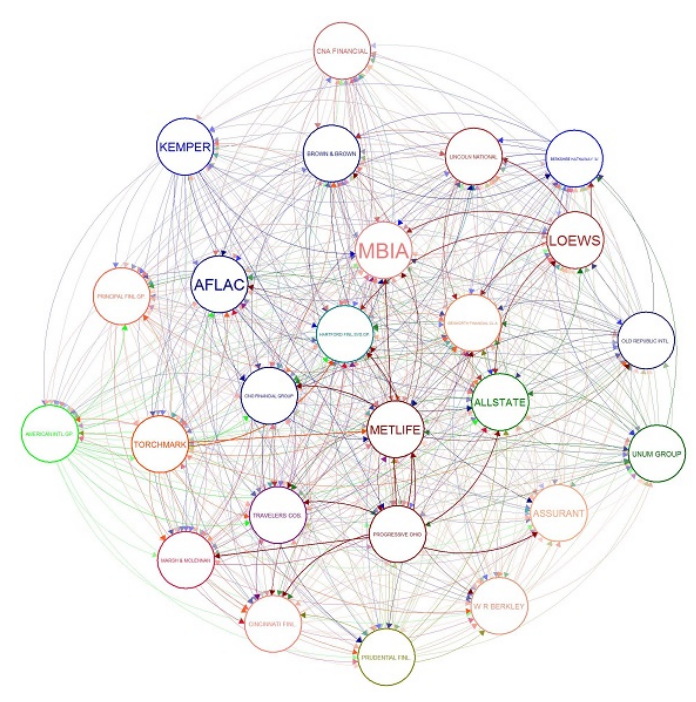

Figure 7: The figure visualizes the network for the Insurers companies dataset. The network is extracted by combining causality network by using quantile regression (QB, Qo and QN) at the $10 \%$ quantile, and the standard granger causality method, during the period 2006-2008. In this case we do not allow the presence of the common factor for network estimations.

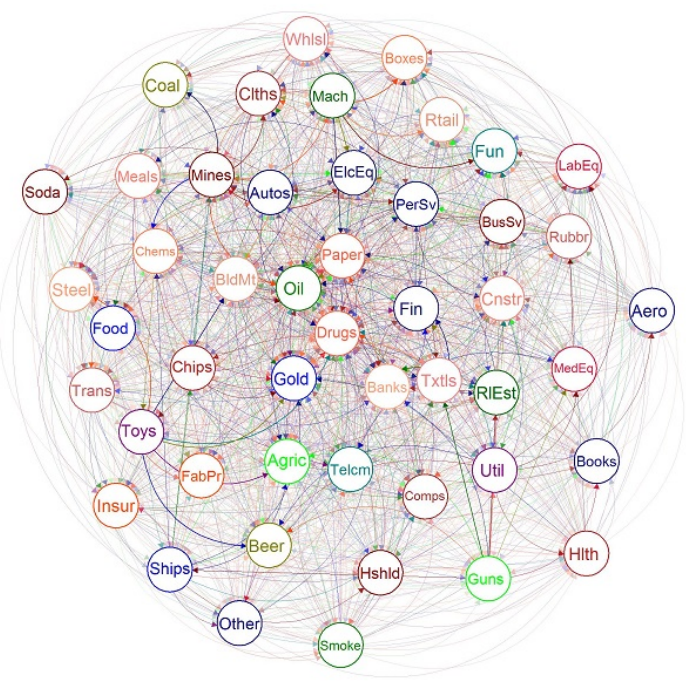

Figure 8: The figure visualizes the network for the Industry portfolios dataset. The network is extracted by combining causality network by using quantile regression (QB, Qo and QN) at the $10 \%$ quantile, and the standard granger causality method, during the period 2006-2008. In this case we do not allow the presence of the common factor for network estimations.

0.1. With the introduction of a plurality of networks, the model fit improves considerably: the residual correlations are centered at zero, with a higher fraction of correlations below - 0.1 , and a marked reduction in the presence of large positive residual correlations. We thus conclude that our approach based on combining several networks within a network augmented linear factor model constitutes an improvement over the use of simple Granger causality. The improvement 
achieved by the latter, over the multifactor model, was already documented in (Billio et al. 2015).

Table 6: The table reports residual correlation descriptive analyses for the Banks dataset. The first column identify the various models, while the second column indicates the number of networks used in the model. In the first column $Q(10 \%)$ identifies the use of a combination of causality networks from quantile regression (QB, Qo and QN) at the $10 \%$ quantile, combined with the Granger causality network. Similarly, when the reference quantile is $50 \%$ or $90 \%$. With $G$ we denote the model using just the Granger casuality network, while the last line refers to the 4-factor CAPM. The table reports statistics for the residuals correlations: the minimum, maximum, the $10 \%$ quantile $q_{10}$, the median $q_{50}$, the $90 \%$ quantile and the number of elements of the correlation matrix lower than -0.1 .

\begin{tabular}{|c|c|c|c|c|c|c|c|c|}
\hline Model & $\begin{array}{c}\mathrm{N} \\
\text { Networks }\end{array}$ & Factor & Min & Max & q10 & q50 & q90 & $\begin{array}{c}\% \text { elements } \\
\leq-0.1\end{array}$ \\
\hline \multicolumn{9}{|c|}{ 2006-2008 } \\
\hline $\mathrm{Q}(10 \%)$ & 4 & $\mathrm{~N}$ & -0.279 & 0.584 & -0.106 & 0.071 & 0.282 & $11.7 \%$ \\
\hline $\mathrm{Q}(50 \%)$ & 4 & $\mathrm{~N}$ & -0.345 & 0.596 & -0.109 & 0.062 & 0.311 & $10.7 \%$ \\
\hline $\mathrm{Q}(90 \%)$ & 4 & $\mathrm{~N}$ & -0.269 & 0.600 & -0.146 & 0.050 & 0.276 & $16.0 \%$ \\
\hline $\mathrm{Q}(10 \%)$ & 4 & $\mathrm{Y}$ & -0.297 & 0.566 & -0.135 & 0.035 & 0.238 & $17.7 \%$ \\
\hline $\mathrm{Q}(50 \%)$ & 4 & $\mathrm{Y}$ & -0.349 & 0.503 & -0.119 & 0.047 & 0.242 & $14.7 \%$ \\
\hline $\mathrm{Q}(90 \%)$ & 4 & $\mathrm{Y}$ & -0.297 & 0.455 & -0.161 & 0.020 & 0.218 & $18.3 \%$ \\
\hline G & 1 & $\mathrm{~N}$ & -0.381 & 0.670 & -0.128 & 0.111 & 0.372 & $13.3 \%$ \\
\hline G & 1 & $\mathrm{Y}$ & -0.394 & 0.641 & -0.128 & 0.089 & 0.378 & $11.7 \%$ \\
\hline 4-F-CAPM & - & - & -0.358 & 0.678 & -0.108 & 0.257 & 0.502 & $10.7 \%$ \\
\hline \multicolumn{9}{|c|}{ 2011-2015 } \\
\hline $\mathrm{Q}(10 \%)$ & 4 & $\mathrm{~N}$ & -0.263 & 0.422 & -0.122 & -0.013 & 0.135 & $15.3 \%$ \\
\hline $\mathrm{Q}(50 \%)$ & 4 & $\mathrm{~N}$ & -0.397 & 0.537 & -0.129 & 0.055 & 0.224 & $13.7 \%$ \\
\hline $\mathrm{Q}(90 \%)$ & 4 & $\mathrm{~N}$ & -0.253 & 0.431 & -0.127 & 0.010 & 0.138 & $15.7 \%$ \\
\hline $\mathrm{Q}(10 \%)$ & 4 & $\mathrm{Y}$ & -0.283 & 0.503 & -0.132 & -0.008 & 0.140 & $19.0 \%$ \\
\hline $\mathrm{Q}(50 \%)$ & 4 & $\mathrm{Y}$ & -0.330 & 0.505 & -0.126 & 0.041 & 0.197 & $14.7 \%$ \\
\hline $\mathrm{Q}(90 \%)$ & 4 & $\mathrm{Y}$ & -0.304 & 0.508 & -0.140 & 0.001 & 0.132 & $17.7 \%$ \\
\hline Granger & 1 & $\mathrm{~N}$ & -0.211 & 0.598 & -0.027 & 0.151 & 0.362 & $3.0 \%$ \\
\hline Granger & 1 & $\mathrm{Y}$ & -0.211 & 0.598 & -0.027 & 0.146 & 0.361 & $3.3 \%$ \\
\hline Multifactor & - & - & -0.122 & 0.669 & 0.054 & 0.266 & 0.487 & $0.3 \%$ \\
\hline
\end{tabular}

For the industry portfolio dataset, the results are less clear, since the various approaches produced very similar findings. This is in line with the heterogeneous results seen for the weights of the various networks, where we were unable to identify a clear preference. We interpret this as evidence to suggest that network augmented linear factor models might usefully improve on traditional linear factor models when we fit the model over single assets and not over portfolios. The aggregation of assets into portfolios probably distorts the dependence structure across assets and limits its impact. There might be a different reason linking the datasets of the banks and insurance companies to the potential presence of a sector-specific factor in the analysis - but if that were the case, we should have seen no such clear improvement in the residual correlations of the composite network cases. We consequently believe that the idea of a missing factor is inconsistent with our findings. Further analyses are needed on this topic, but we leave them to 
Table 7: The table reports residual correlation descriptive analyses for the Insurance Companies dataset. The first column identify the various models, while the second column indicates the number of networks used in the model. In the first column $Q(10 \%)$ identifies the use of a combination of causality networks from quantile regression (QB, Qo and QN) at the $10 \%$ quantile, combined with the Granger causality network. Similarly, when the reference quantile is 50\% or $90 \%$. With $G$ we denote the model using just the Granger casuality network, while the last line refers to the 4-factor CAPM. The table reports statistics for the residuals correlations: the minimum, maximum, the $10 \%$ quantile $q_{10}$, the median $q_{50}$, the $90 \%$ quantile and the number of elements of the correlation matrix lower than -0.1 .

\begin{tabular}{ccccccccc}
\hline Model & $\begin{array}{c}\text { N } \\
\text { Networks }\end{array}$ & Factor & Min & Max & q10 & q50 & q90 & $\begin{array}{c}\text { \% elements } \\
\leq-0.1\end{array}$ \\
\hline & & & & & & & \\
\hline Q(10\%) & 4 & N & -0.437 & 0.686 & -0.205 & 0.003 & 0.250 & $27.7 \%$ \\
Q(50\%) & 4 & N & -0.429 & 0.669 & -0.167 & 0.039 & 0.258 & $15.3 \%$ \\
Q(90\%) & 4 & N & -0.448 & 0.676 & -0.207 & 0.009 & 0.255 & $25.7 \%$ \\
Q(10\%) & 4 & Y & -0.436 & 0.684 & -0.203 & 0.004 & 0.246 & $28.0 \%$ \\
Q(50\%) & 4 & Y & -0.417 & 0.473 & -0.176 & 0.023 & 0.226 & $19.3 \%$ \\
Q(90\%) & 4 & Y & -0.464 & 0.671 & -0.214 & 0.001 & 0.253 & $27.0 \%$ \\
G & 1 & N & -0.466 & 0.831 & -0.166 & 0.044 & 0.316 & $17.7 \%$ \\
G & 1 & Y & -0.460 & 0.714 & -0.166 & 0.048 & 0.323 & $15.0 \%$ \\
$4-F-C A P M$ & - & - & -0.370 & 0.847 & -0.149 & 0.089 & 0.422 & $14.0 \%$ \\
\hline & & & $\mathbf{2 0 1 1 - 2 0 1 5}$ & & & & \\
\hline Q(10\%) & 4 & N & -0.290 & 0.504 & -0.121 & 0.009 & 0.157 & $14.7 \%$ \\
Q(50\%) & 4 & N & -0.258 & 0.548 & -0.149 & 0.019 & 0.202 & $16.7 \%$ \\
Q(90\%) & 4 & N & -0.289 & 0.348 & -0.137 & -0.006 & 0.167 & $19.3 \%$ \\
Q(10\%) & 4 & Y & -0.333 & 0.503 & -0.153 & -0.012 & 0.189 & $20.7 \%$ \\
Q(50\%) & 4 & Y & -0.267 & 0.592 & -0.156 & 0.016 & 0.192 & $18.3 \%$ \\
Q(90\%) & 4 & Y & -0.299 & 0.398 & -0.152 & -0.001 & 0.199 & $21.3 \%$ \\
G & 1 & N & -0.136 & 0.658 & -0.005 & 0.121 & 0.317 & $1.7 \%$ \\
G & 1 & Y & -0.136 & 0.658 & -0.014 & 0.116 & 0.315 & $1.7 \%$ \\
$4-F-C A P M$ & - & - & -0.103 & 0.658 & 0.017 & 0.138 & 0.332 & $0.3 \%$ \\
\hline
\end{tabular}

future research.

We close this section with a few comments on the coefficients for monitoring the impact of the composite networks on the various assets, as included in the diagonal of matrix $\mathcal{R}$ in equation (5). Appendix 1 shows the plots of the coefficients for each bank, insurance company and industrial portfolio, across the various combined networks. Overall, we note that the coefficients are usually positive and significant for banks and insurance companies, while the networks impact is more heterogeneous and of limited significance for industrial portfolios. These results deserve a more thorough analysis, but this goes beyond the scope of the present paper.

\subsection{Robustness checks}

The previously-reported results focus on the combination of all four different networks, where the model providing the optimal combination makes use of weekly data and allows for a heterogeneous impact of the composite network on the assets. Here we provide some additional 
Table 8: The table reports residual correlation descriptive analyses for the Industry portfolios dataset. The first column identify the various models, while the second column indicates the number of networks used in the model. In the first column $Q(10 \%)$ identifies the use of a combination of causality networks from quantile regression (QB, Qo and QN) at the $10 \%$ quantile, combined with the Granger causality network. Similarly, when the reference quantile is 50\% or $90 \%$. With $G$ we denote the model using just the Granger casuality network, while the last line refers to the 4-factor CAPM. The table reports statistics for the residuals correlations: the minimum, maximum, the $10 \%$ quantile $q_{10}$, the median $q_{50}$, the $90 \%$ quantile and the number of elements of the correlation matrix lower than -0.1 .

\begin{tabular}{ccccccccc}
\hline Model & $\begin{array}{c}\text { N } \\
\text { Networks }\end{array}$ & Factor & Min & Max & q10 & q50 & q90 & $\begin{array}{c}\text { \% elements } \\
\leq-0.1\end{array}$ \\
\hline & & & & & & & \\
\hline Q(10\%) & 4 & N & -0.465 & 0.611 & -0.191 & -0.001 & 0.201 & $25.6 \%$ \\
Q(50\%) & 4 & N & -0.369 & 0.663 & -0.161 & 0.018 & 0.249 & $20.6 \%$ \\
Q(90\%) & 4 & N & -0.443 & 0.608 & -0.195 & -0.006 & 0.206 & $25.9 \%$ \\
Q(10\%) & 4 & Y & -0.417 & 0.625 & -0.187 & 0.006 & 0.220 & $24.1 \%$ \\
Q(50\%) & 4 & Y & -0.454 & 0.627 & -0.159 & 0.011 & 0.233 & $20.4 \%$ \\
Q(90\%) & 4 & Y & -0.508 & 0.592 & -0.187 & -0.007 & 0.215 & $23.8 \%$ \\
G & 1 & N & -0.384 & 0.687 & -0.146 & 0.025 & 0.257 & $18.6 \%$ \\
G & 1 & Y & -0.401 & 0.687 & -0.157 & 0.011 & 0.239 & $19.8 \%$ \\
$4-F-C A P M$ & - & - & -0.486 & 0.733 & -0.219 & 0.003 & 0.267 & $27.1 \%$ \\
\hline & & & $\mathbf{2 0 1 1 - 2 0 1 5}$ & & & & \\
\hline Q(10\%) & 4 & N & -0.463 & 0.567 & -0.140 & -0.008 & 0.135 & $19.7 \%$ \\
Q(50\%) & 4 & N & -0.454 & 0.518 & -0.136 & 0.005 & 0.169 & $16.7 \%$ \\
Q(90\%) & 4 & N & -0.471 & 0.572 & -0.144 & -0.013 & 0.139 & $19.9 \%$ \\
Q(10\%) & 4 & Y & -0.432 & 0.497 & -0.147 & -0.004 & 0.148 & $18.9 \%$ \\
Q(50\%) & 4 & Y & -0.470 & 0.511 & -0.135 & 0.001 & 0.155 & $16.6 \%$ \\
Q(90\%) & 4 & Y & -0.438 & 0.565 & -0.143 & -0.006 & 0.154 & $17.9 \%$ \\
G & 1 & N & -0.470 & 0.577 & -0.140 & 0.007 & 0.199 & $18.0 \%$ \\
G & 1 & Y & -0.503 & 0.577 & -0.139 & 0.006 & 0.177 & $17.7 \%$ \\
$4-F-C A P M$ & - & - & -0.456 & 0.592 & -0.147 & 0.007 & 0.208 & $18.4 \%$ \\
\hline
\end{tabular}

comments on variations to the model-based combination design. In particular, we estimate the model on monthly data, we consider combining just three networks, and we control for the optimal combination when the network's impact is homogeneous across assets.

Section F contains tables assessing the model-based network combination when we exclude a network. We consider two specific cases: the first excludes the network estimated from Granger causality in order to highlight the relevance of competing quantile causality networks; the second disregards the less relevant quantile causality network (the so-called baseline quantile causality). All the analyses are based on weekly data. The results confirm the relevance of the non-parametric approach for the purpose of obtaining a quantile causality that, in several cases, generates the largest and statistically most significant coefficients. The baseline quantile causality is the least relevant, even when the Granger causality network is excluded from the layers. The results for the industry portfolios are the most heterogeneous in terms of network relevance, and they show little improvement with respect to the contraction of the residual correlations. 
For the banks and insurance companies, there is a marked gain in moving from linear factor models, or Granger causality augmented factor models, to a model that accounts for the presence of quantile causality; the average residual correlation decreases considerably.

Section $\mathrm{G}$ contains estimates of the network combination modelled on monthly data (as opposed to the weekly data considered in the previous section) ${ }^{6}$ We confirmed the importance of non-parametric quantile causality and the limited impact of Granger causality. Once again, the outcome is clearer for the datasets concerning banks and insurance companies than the industry portfolio dataset. In addition, contrary to the evidence emerging from the weekly data, the contraction on the residual correlations for the insurance company dataset is less evident during the financial crisis, while it is striking in the second period.

Given the heterogeneity of the network's impact on returns, as mentioned at the end of the previous section, we check the model's performance when we impose a common reaction of the returns to exposure to the network. Section $\mathrm{H}$ illustrates the outcomes for the composite network and the impact of the network on returns in the three datasets and two sample periods, when the model is estimated on weekly data. The only notable changes concern the composite network parameters: we now find a larger number of statistically significant coefficients. In addition, Granger causality seems to become more relevant, in the insurance companies dataset at least. The non-parametric quantile causality network nonetheless retains its role and receives the highest coefficients. Moving to the model-based combination confirms the previous results and enables a reduction in the average residual correlations in the banks and insurance companies datasets.

\section{Concluding remarks}

Causality networks have attracted some attention in the financial economics literature in recent years for the purposes of systemic risk interpretation. We show that the structure of causality networks changes considerably if we move from the mean, commonly associated with traditional Granger causality, to the quantiles. In the latter case, the causality across assets can be measured by means of quantile regression-based approaches. Here we illustrate three different possibilities: the simple quantile regression, its generalization to the quantile-on-quantile approach of Sim and Zhou (2015) and the non-parametric test due to Jeong et al. (2012). We show that changing the approach to network estimation gives rise to networks in which a systemic risk interpretation points to the existence of dense networks with a broader systemic relevance. By focusing on a linear factor model augmented with a multi-layer network dependence, we also demonstrate that causality networks are useful for capturing this dependence across financial returns, going beyond what can be explained by a market factor. This paves the way to further analyses, and has an associated impact on diversification analyses and portfolio construction, as well as on asset pricing.

\footnotetext{
${ }^{6}$ In this case too, we ran a sensitivity analysis on the effects of excluding a network. The results are available on request.
} 


\section{References}

Acemoglu, D., Carvalho, D. M., and Ozdaglar, A. (2012). The network origins of aggregate fluctuations. Econometrica, 80(5):1977-2016.

Anselin, L. (1988). Spatial Econometrics: Methods and Models. Springer Netherlands.

Bargigli, L., di Iasio, G., Infante, L., Lillo, F., and Pierobon, F. (2015). The multiplex structure of interbank networks. Quantitative Finance, 15(4):673-691.

Barigozzi, M. and Brownlees, C. (2014). Nets: Network estimation for time series. Working Papers 723, Barcelona Graduate School of Economics.

Billio, M., Caporin, M., Frattarolo, L., and Pelizzon, L. (2016). Networks in risk spillovers: A multivariate garch perspective. University Ca' Foscari of Venice, Dept. of Economics Research Paper Series No. No. 03/WP/ 2016.

Billio, M., Caporin, M., Panzica, R., and Pelizzon, L. (2015). The impact of network connectivity on factor exposures, asset pricing and portfolio diversification. Mimeo.

Billio, M., Getmansky, M., Lo, A. W., and Pelizzon, L. (2012). Econometric measures of connectedness and systemic risk in the finance and insurance sectors. Journal of Financial Economics, 104(3):535-559.

Billio, M., Gray, D., Getmansky, M., Lo, A., Merton, R., and Pelizzon, L. (2014). Sovereign, bank and insurance credit spreads: Connectedness and system networks. MIT Working Paper.

Bonacich, P. (1987). Power and centrality: A family of measures. American journal of sociology, pages $1170-1182$.

Brandsma, A. S. and Ketellapper, R. H. (1979). A biparametric approach to spatial autocorrelation. Environment and Planning A, 11:51-58.

Candelon, B. and Topkavi, S. (2016). A nonparametric test for granger causality in distribution with application to financial contagion. Journal of Business and Economic Statistics, $34(2): 240-253$.

Caporin, M. and Paruolo, P. (2015). Proximity-structured multivariate volatility models. Econometric Reviews, 34(5):559-593.

Carhart, M. (1997). On the persistence of mutual fund performance. Journal of Finance, $52: 57-82$.

Cheung, Y. W. and Ng, L. K. (1996). A causality-in-variance test and its application to financial market prices. Journal of Econometrics, 72:33-48.

Comte, F. and Lieberman, O. (2000). Second-order noncausality in multivariate garch processes. Journal of Time Series Analysis, 21(5):535-557. 
Cont, R. (2001). Empirical properties of asset returns: stylized facts and statistical issues. Quantitative Finance, 1:223-236.

Corsi, F., Lillo, F., and Pirino, D. (2015). Measuring flight-to-quality with granger-causality tail risk networks. Available at SSRN 2576078.

Diebold, F. X. and Yilmaz, K. (2014). On the network topology of variance decompositions: measuring the connectedness of financial firms. Journal of Econometrics, 182(1):119-134.

Diebold, F. X. and Yilmaz, K. (2015). Financial and Macroeconomic Connectedness. Oxford University Press.

Elhorst, J. P., Lacombe, D. J., and Piras, G. (2012). On model specification and parameter space definitions in higher order spatial econometric models. Regional Science and Urban Economics, 42(1):211-220.

Engle, R. F. and Manganelli, S. (2004). Caviar: Conditional autoregressive value at risk by regression quantiles. Journal of Business $8 \mathcal{G}$ Economic Statistics, American Statistical Association, 22:367-381.

Fama, E. and French, K. (1993). Common risk factors in the returns of stocks and bonds. Journal of Financial Economics, 33(3-53).

Fama, E. and French, K. (1995). Size and book-to-market factors in earnings and returns. Journal of Finance, 50(1):131-155.

Fruchterman, T. M. and Reingold, E. M. (1991). Graph drawing by force-directed placement. Software: Practice and experience, 21(11):1129-1164.

Geweke, J. (1984). Inference and causality in economic time series. In Handbook of Econometrics, 2:1101-1144.

Granger, C. W. J. (1969). Investigating causal relations by econometric models and cross spectral methods. Econometrica, 37:424-438.

Granger, C. W. J. (1980). Testing for causality: a personal viewpoint. Journal of Economics Dynamic and Control, 2(4):329-352.

Granger, C. W. J. (1988). Some recent developments in a concept of causality. Journal of Econometrics, 39:199-211.

Granger, C. W. J., Robins, R. P., and Engle, R. F. (1986). Wholesale retail prices: bivariate time series modeling with forecastable error variances. In Model reliability. Boston MA: MIT Press, pages 1-17.

Hafner, C. M. (2003). Fourth moments of multivariate garch models. Journal of Financial Econometrics, 1(1):26-54.

Hafner, C. M. and Herwartz, H. (2008). Testing for causality in variance using multivariate garch models. Annales d'Économie et de Statistique, 89:215-241. 
Hautsch, N., Kyj, L. M., and Malec, P. (2013). Do high-frequency data improve highdimensional portfolio allocations? Technical report, Sonderforschungsbereich 649, Humboldt University, Berlin, Germany.

Hautsch, N., Kyj, L. M., and Oomen, R. C. (2012). A blocking and regularization approach to high-dimensional realized covariance estimation. Journal of Applied Econometrics, 27(4):625645 .

Hautsch, N., Schaumburg, J., and Schienle, M. (2014). Forecasting systemic impact in financial networks. International Journal of Forecasting, 30(3):781-794.

Hautsch, N., Schaumburg, J., and Schienle, M. (2015). Financial network systemic risk contributions. Review of Finance, 19(2):685-738.

Hong, Y., Liu, Y., and S., W. (2009). Granger causality in risk and detection of extreme risk spillover between financial markets. Journal of Econometrics, 150:271-287.

Hoover, K. D. (2001). Causality in Macroeconomics. Cambridge University Press.

Jeong, K., Hardle, W. K., and Song, S. (2012). A consistent nonparametric test for causality in quantile. Econometric Theory, 28:861-887.

Kelly, B., Lustig, H., and Nieuwerburgh, S. V. (2013). Firm volatility in granular networks. Mimeo. Booth School of Business, UCLA-Anderson and Stern School of Business.

Koenker, R. (2005). Quantile Regression. New York: Cambridge University Press.

Koenker, R. and Bassett, G. (1978). Regression quantiles. Econometrica, 46(1):33-50.

Koenker, R. and Bassett, G. (1982). Robust tests for heteroscedasticity based on regression quantiles. Econometrica, 50:43-61.

Lee, L. F. and Liu, X. (2010). Efficient gmm estimation of higher order spatial autoregressive models with autoregressive distrurbances. Econometric Theory, 26:187-230.

Lee, T. and Yang, W. (2014). Granger-causality in quantiles between financial markets: Using copula approach. International Review of Financial Analysis, 33:70-78.

Léon, C., Berndsen, R., and Renneboog, L. (2014). Financial stability and interacting networks of financial institutions and market infrastructures. CentER discussionpaper Series, 69.

LeSage, J. and Pace, R. K. (2009). Introduction to Spatial Econometrics. Boca Raton, FL, USA, Chapman and Hall/CRC, Taylor and Francs Group.

Montagna, M. and Kok, C. (2013). Multi-layered interbank model for assessing systemic risk. Kiel Institute for the World Economy Working Paper No. 1873 and ECB Working Paper.

Newman, M. (2010). Networks: an introduction. Oxford university press.

Newman, M. E. (2002). Assortative mixing in networks. Physical review letters, 89(20):208701. 
Newman, M. E. (2003). Mixing patterns in networks. Physical Review E, 67(2):026126.

Ozdagli, A. and Weber, M. (2015). Monetary policy through production networks: Evidence from the stock market. Working Paper, University of Chicago, Chicago, IL.

Sim, N. and Zhou, H. (2015). Oil prices, us stock return, and the dependence between their quantiles. Journal of Banking $\&$ Finance, 55:1-8.

Van Mieghem, P. (2010). Graph spectra for complex networks. Cambridge University Press.

Wasserman, S. and Faust, K. (1994). Social network analysis: Methods and applications, volume 8. Cambridge university press.

White, H., Kim, T., and Manganelli, S. (2015). VAR for VaR: Measuring tail dependence using multivariate regression quantiles. Journal of Econometrics, 187:169-188. 


\section{Appendix}

\section{A Banks and Insurance companies}

In the empirical analyses we use the bank or the insurance companies as in the following lists. Within parentheses we report the ticker. A single star * identifies companies included only in the first sample - 2006 to 2008 - while a double star identifies companies included only in the second sample - 2011-2015. When stars are not present, the companies are included in both samples.

\section{Banks:}

Astoria Financial Corporation (AF*), Associated Banc-Corp (ASB), Bank of America (BAC), Bancfirst Corporation (BANF*), Credicorp (BAP), BB\&T (BBT), Bbx Capital Corporation $\left(\mathrm{BBX}^{\star}\right)$, Bank Mutual Corporation (BKMU $\left.{ }^{\star}\right)$, Bank of Hawaii $(\mathrm{BOH})$, Bok Financial Corporation (BOKF), Boston Private Financial Holdings $\left(\mathrm{BPFH}^{\star}\right)$, Brookline Bancorp (BRKL $\left.{ }^{\star}\right)$, Bancorpsouth $\left(\mathrm{BXS}^{\star}\right)$, Citigroup (C), Cathay General Bancorp $\left(\mathrm{CATY}^{\star}\right)$, Commerce Bancshares (CBSH), Capitol Federal Financial (CFFN), Cullen/Frost Bankers (CFR), Chemical Financial Corporation $\left(\mathrm{CHFC}^{\star}\right)$, Comerica (CMA), City National (CN), Central Pacific Financial Corporation $\left(\mathrm{CPF}^{\star}\right)$, CVB Financial Corporation $\left(\mathrm{CVBF}^{\star}\right)$, Doral Financial Corporation $\left(\mathrm{DRLCQ}^{\star}\right)$, East West Bancorp (EWBC), First Horizon National $\left(\right.$ FHN $\left.^{\star \star}\right)$, Fifth Third Bancorp (FITB ${ }^{\star \star}$ ), Firstmerit $\left(\right.$ FMER $\left.^{\star \star}\right)$, First Niagara Financial Group (FNFG $\left.{ }^{\star \star}\right)$, First Republic Bank $\left(\right.$ FRC $\left.^{\star \star}\right)$, Fulton Financial Corporation (FULT ${ }^{\star \star}$ ), Huntington Bancshares Incorporated (HBAN ${ }^{\star \star}$ ), Hudson City Bancorp $\left(\mathrm{HCB}^{\star \star}\right)$, Investors Bancorp $\left(\mathrm{ISBC}^{\star \star}\right)$, Jp Morgan Chase \& Co. (JPM $\left.{ }^{\star \star}\right)$, Keycorp $\left(\mathrm{KEY}^{\star \star}\right), \mathrm{M} \& \mathrm{~T}$ Bank $\left(\mathrm{MTB}^{\star \star}\right)$.

\section{Insurances:}

American Financial Group (AFG ${ }^{\star \star}$ ), Aflac (AFL), American International Group (AIG), Assurant (AIZ), The Allstate Corporation (ALL), Berkshire Hathaway (BRKA), Brown \& Brown (BRO), Cincinnati Financial Corporation (CINF), Cna Financial Corporation (CNA), Cno Financial Group $\left(\mathrm{CNO}^{\star}\right)$, Erie Indemnity Company (ERIE $\left.{ }^{\star \star}\right)$, Genworth Financial (GNW), The Hartford Financial Services Group (HIG), Kemper $\left(\mathrm{KMPR}^{\star}\right)$, Loews (L), Lincoln National (LNC), Mbia (MBI*), Metlife (MET), Markel (MKL $\left.{ }^{\star \star}\right)$, Marsh \& Mclennan (MMC), Old Republic International Corporation $\left(\mathrm{ORI}^{\star}\right)$, Principal Financial Group (PFG), Progressive Ohio (PGR), Prudential Financial (PRU), Reinsurance Group of America (RGA ${ }^{\star \star}$ ), Torchmark (TMK), The Travelers Companies (TRV), Unum Group (UNM), W.R. Berkley Corporation (WRB).

\section{B Descriptive statistics}




\begin{tabular}{|c|c|c|c|c|c|c|c|c|c|c|c|c|c|c|c|c|c|}
\hline & STOCK & MEAN & MED & ST DEV & MIN & MAX & $1^{s t} \mathrm{Q}$ & $3^{\text {rd }} \mathrm{Q}$ & SKEW & KURT & $L B_{1}$ & $L B_{5}$ & $L B_{10}$ & $S L B_{1}$ & $S L B_{5}$ & $S L B_{10}$ & MV \\
\hline & $\mathrm{C}$ & -0.262 & -0.127 & 4.296 & -30.661 & 45.632 & -1.113 & 0.711 & 0.673 & 30.033 & 5.716 & 0.000 & 0.002 & 0.000 & 0.000 & 0.000 & 236630.90 \\
\hline & $\mathrm{BAC}$ & -0.157 & -0.039 & 3.765 & -30.416 & 24.060 & -0.977 & 0.688 & -0.468 & 19.555 & 72.686 & 0.295 & 0.126 & 0.000 & 0.000 & 0.000 & 212543.10 \\
\hline & BBT & -0.056 & -0.070 & 3.108 & -26.608 & 21.198 & -1.167 & 0.849 & -0.094 & 18.219 & 0.326 & 1.159 & 0.000 & 0.000 & 0.000 & 0.000 & 21015.19 \\
\hline & CMA & -0.139 & -0.102 & 3.361 & -20.720 & 18.805 & -1.266 & 0.885 & 0.049 & 10.555 & 23.288 & 6.167 & 0.032 & 0.000 & 0.000 & 0.000 & 9392.46 \\
\hline & ASB & -0.058 & -0.030 & 2.905 & -18.999 & 19.354 & -0.916 & 0.705 & 0.076 & 14.517 & 94.558 & 0.339 & 0.009 & 0.107 & 0.000 & 0.000 & 4619.78 \\
\hline & $\mathrm{CN}$ & -0.053 & 0.000 & 2.730 & -17.379 & 20.213 & -0.964 & 0.827 & 0.048 & 13.119 & 0.080 & 0.035 & 0.003 & 0.000 & 0.000 & 0.000 & 3763.18 \\
\hline & $\mathrm{CBSH}$ & -0.003 & -0.040 & 2.113 & -12.894 & 15.113 & -0.808 & 0.651 & 0.696 & 13.581 & 0.000 & 0.000 & 0.000 & 0.000 & 0.000 & 0.000 & 3428.85 \\
\hline & $\mathrm{AF}$ & -0.077 & -0.096 & 2.676 & -18.972 & 13.698 & -1.172 & 0.820 & -0.251 & 11.170 & 0.054 & 0.032 & 0.011 & 0.000 & 0.000 & 0.000 & 3162.41 \\
\hline & BOKF & -0.016 & -0.018 & 2.228 & -13.426 & 12.315 & -0.848 & 0.764 & 0.055 & 12.242 & 63.004 & 0.283 & 0.052 & 0.000 & 0.000 & 0.000 & 3135.55 \\
\hline & CFR & -0.008 & -0.051 & 2.302 & -13.183 & 16.306 & -0.915 & 0.852 & 0.539 & 13.505 & 0.007 & 0.000 & 0.000 & 0.000 & 0.000 & 0.000 & 2853.67 \\
\hline \multirow{15}{*}{$\Delta$} & $\mathrm{BOH}$ & -0.017 & -0.019 & 2.458 & -25.508 & 12.946 & -0.859 & 0.834 & -1.279 & 23.778 & 0.009 & 0.002 & 0.006 & 0.000 & 0.000 & 0.000 & 2695.89 \\
\hline & $\mathrm{BAP}$ & 0.104 & 0.089 & 2.572 & -17.439 & 11.226 & -1.017 & 1.368 & -0.507 & 8.735 & 76.155 & 6.593 & 1.739 & 0.000 & 0.000 & 0.000 & 2572.86 \\
\hline & CFFN & 0.043 & 0.052 & 1.636 & -7.664 & 6.606 & -0.619 & 0.755 & -0.127 & 6.254 & 10.379 & 14.081 & 0.059 & 0.000 & 0.000 & 0.000 & 2366.73 \\
\hline & EWBC & -0.109 & -0.155 & 3.730 & -23.669 & 24.166 & -1.255 & 0.869 & 0.263 & 12.930 & 47.310 & 11.160 & 19.360 & 0.000 & 0.000 & 0.000 & 2089.00 \\
\hline & BXS & 0.008 & -0.077 & 2.854 & -15.116 & 19.711 & -1.165 & 1.088 & 0.592 & 11.463 & 6.615 & 0.065 & 0.173 & 0.000 & 0.000 & 0.000 & 1895.44 \\
\hline & CATY & -0.055 & -0.121 & 3.631 & -23.886 & 21.314 & -1.504 & 1.227 & 0.167 & 11.957 & 39.151 & 1.648 & 1.971 & 0.000 & 0.000 & 0.000 & 1865.30 \\
\hline & CVBF & -0.029 & -0.145 & 3.257 & -18.965 & 20.210 & -1.419 & 1.101 & 0.702 & 10.139 & 1.833 & 1.097 & 3.680 & 0.000 & 0.000 & 0.000 & 1241.16 \\
\hline & BPFH & -0.198 & -0.138 & 3.671 & -30.902 & 17.640 & -1.403 & 1.161 & -1.226 & 17.170 & 13.155 & 2.258 & 2.606 & 0.007 & 0.001 & 0.000 & 1171.49 \\
\hline & DRLCQ & -0.443 & -0.522 & 6.085 & -36.564 & 36.891 & -2.885 & 1.968 & -0.166 & 9.962 & 84.586 & 85.266 & 69.825 & 1.543 & 0.144 & 0.000 & 1097.65 \\
\hline & $\mathrm{CPF}$ & -0.169 & -0.199 & 3.925 & -22.119 & 29.376 & -1.668 & 1.259 & 0.553 & 12.079 & 99.950 & 0.257 & 1.170 & 0.000 & 0.000 & 0.000 & 1042.41 \\
\hline & BRKL & -0.038 & -0.076 & 2.461 & -14.973 & 11.878 & -1.119 & 0.990 & 0.158 & 7.816 & 0.088 & 0.175 & 0.259 & 0.000 & 0.000 & 0.000 & 918.22 \\
\hline & BBX & -0.330 & -0.235 & 6.086 & -48.290 & 35.188 & -2.062 & 1.308 & -0.797 & 16.628 & 15.046 & 30.970 & 3.020 & 0.000 & 0.000 & 0.000 & 828.74 \\
\hline & CHFC & -0.017 & -0.034 & 2.904 & -19.297 & 17.823 & -1.368 & 1.131 & 0.267 & 10.009 & 0.173 & 1.038 & 6.583 & 0.035 & 0.000 & 0.000 & 775.59 \\
\hline & BKMU & 0.011 & -0.080 & 1.970 & -14.235 & 9.307 & -0.844 & 0.917 & -0.048 & 9.507 & 0.000 & 0.000 & 0.000 & 0.076 & 0.000 & 0.000 & 718.23 \\
\hline & $\mathrm{BANF}$ & 0.039 & 0.000 & 2.709 & -24.878 & 21.243 & -1.325 & 1.233 & 0.039 & 19.231 & 0.158 & 0.044 & 0.667 & 0.000 & 0.000 & 0.000 & 675.88 \\
\hline
\end{tabular}

Table B.9: Descriptive statistics of the daily returns generated by the 25 U.S. banks with the largest market value, as recorded at the beginning of 2006, in the period between January 3, 2006 and December 31, 2008. From left to right the table reports, for each stock, the mean (\%), the median $(\%)$, the standard deviation (\%), the minimum (\%), the maximum (\%), the first and the third quartiles (\%), the skewness, the kurtosis, the p-value of the Ljung-Box test (\%), at the significance level of the $5 \%$, applied for both the returns $(L B)$ and the squared returns $(S L B)$ with lags equal to 1,5 and 10, respectively, and the market value recorded in January 2006. 


\begin{tabular}{|c|c|c|c|c|c|c|c|c|c|c|c|c|c|c|c|c|}
\hline STOCK & MEAN & MED & ST DEV & MIN & MAX & $1^{s t} \mathrm{Q}$ & $3^{\text {rd }} \mathrm{Q}$ & SKEW & KURT & $L B_{1}$ & $L B_{5}$ & $L B_{10}$ & $S L B_{1}$ & $S L B_{5}$ & $S L B_{10}$ & MV \\
\hline JPM & 0.035 & 0.048 & 1.698 & -9.888 & 8.101 & -0.789 & 0.901 & -0.206 & 7.043 & 0.338 & 0.628 & 0.272 & 0.000 & 0.000 & 0.000 & 186018.60 \\
\hline $\mathrm{BAC}$ & 0.018 & 0.000 & 2.295 & -22.713 & 15.481 & -1.050 & 1.103 & -0.508 & 14.427 & 1.340 & 0.000 & 0.000 & 0.000 & 0.000 & 0.000 & 136331.90 \\
\hline $\mathrm{C}$ & 0.007 & 0.000 & 2.129 & -17.934 & 12.968 & -0.944 & 0.981 & -0.533 & 10.310 & 8.722 & 0.001 & 0.001 & 0.000 & 0.000 & 0.000 & 132889.30 \\
\hline BBT & 0.029 & 0.065 & 1.497 & -11.274 & 6.693 & -0.716 & 0.890 & -0.685 & 8.638 & 0.001 & 0.000 & 0.000 & 0.000 & 0.000 & 0.000 & 18978.31 \\
\hline FITB & 0.025 & 0.106 & 1.736 & -12.067 & 8.709 & -0.817 & 0.908 & -0.430 & 8.619 & 0.001 & 0.000 & 0.000 & 0.000 & 0.000 & 0.000 & 12549.82 \\
\hline MTB & 0.026 & 0.034 & 1.355 & -8.059 & 6.554 & -0.624 & 0.732 & -0.222 & 6.892 & 0.008 & 0.000 & 0.001 & 0.000 & 0.000 & 0.000 & 10488.55 \\
\hline KEY & 0.032 & 0.074 & 1.807 & -10.987 & 8.319 & -0.906 & 1.031 & -0.228 & 7.187 & 0.000 & 0.000 & 0.000 & 0.000 & 0.000 & 0.000 & 8339.36 \\
\hline BAP & -0.016 & -0.009 & 1.711 & -20.843 & 6.231 & -0.918 & 0.894 & -1.487 & 21.228 & 41.290 & 15.174 & 10.460 & 9.994 & 0.071 & 1.654 & 7814.11 \\
\hline CMA & -0.001 & 0.041 & 1.772 & -11.118 & 6.100 & -0.879 & 0.942 & -0.701 & 7.149 & 18.082 & 0.247 & 0.554 & 0.000 & 0.000 & 0.000 & 6795.13 \\
\hline HBAN & 0.038 & 0.102 & 1.794 & -10.472 & 8.542 & -0.931 & 1.059 & -0.301 & 6.869 & 0.000 & 0.000 & 0.000 & 0.000 & 0.000 & 0.000 & 5750.23 \\
\hline $\mathrm{HCB}$ & -0.018 & 0.000 & 1.805 & -11.304 & 14.569 & -0.748 & 0.793 & -0.325 & 11.772 & 0.010 & 0.178 & 1.702 & 0.001 & 0.000 & 0.000 & 5161.57 \\
\hline FRC & 0.065 & 0.064 & 1.546 & -16.359 & 8.376 & -0.715 & 0.921 & -0.901 & 15.518 & 0.004 & 0.016 & 0.081 & 0.064 & 0.013 & 0.047 & 3821.80 \\
\hline CFR & -0.001 & 0.066 & 1.396 & -8.121 & 7.493 & -0.677 & 0.758 & -0.102 & 6.964 & 0.004 & 0.000 & 0.001 & 0.000 & 0.000 & 0.000 & 3667.12 \\
\hline BOKF & 0.009 & 0.059 & 1.403 & -9.594 & 6.062 & -0.709 & 0.720 & -0.390 & 7.708 & 0.005 & 0.003 & 0.097 & 0.000 & 0.000 & 0.000 & 3553.32 \\
\hline $\mathrm{CBSH}$ & 0.025 & 0.090 & 1.316 & -6.635 & 6.047 & -0.697 & 0.776 & -0.307 & 5.971 & 0.002 & 0.001 & 0.004 & 0.000 & 0.000 & 0.000 & 3552.96 \\
\hline EWBC & 0.060 & 0.091 & 1.747 & -9.353 & 8.752 & -0.867 & 0.992 & -0.158 & 6.443 & 0.412 & 0.009 & 0.000 & 0.000 & 0.000 & 0.000 & 3321.22 \\
\hline $\mathrm{CN}$ & 0.030 & 0.000 & 1.577 & -8.200 & 17.330 & -0.687 & 0.821 & 0.850 & 17.467 & 6.143 & 0.012 & 0.009 & 8.723 & 0.002 & 0.000 & 3083.03 \\
\hline FHN & 0.017 & 0.032 & 1.884 & -11.185 & 7.559 & -1.003 & 1.034 & -0.415 & 6.255 & 6.398 & 0.799 & 2.454 & 0.000 & 0.000 & 0.000 & 2999.39 \\
\hline FNFG & -0.020 & 0.017 & 1.708 & -14.511 & 13.548 & -0.878 & 0.922 & -0.556 & 13.534 & 3.662 & 2.318 & 0.807 & 4.568 & 2.718 & 1.949 & 2907.62 \\
\hline ASB & 0.017 & 0.059 & 1.666 & -12.420 & 7.165 & -0.858 & 1.005 & -0.669 & 8.529 & 0.003 & 0.000 & 0.000 & 0.000 & 0.000 & 0.000 & 2538.15 \\
\hline $\mathrm{BOH}$ & 0.023 & 0.085 & 1.323 & -8.229 & 5.795 & -0.671 & 0.799 & -0.296 & 6.328 & 0.027 & 0.005 & 0.031 & 0.000 & 0.000 & 0.000 & 2272.40 \\
\hline FULT & 0.018 & 0.085 & 1.678 & -10.064 & 8.031 & -0.806 & 0.913 & -0.389 & 7.399 & 0.006 & 0.000 & 0.001 & 0.000 & 0.000 & 0.000 & 2216.42 \\
\hline CFFN & 0.004 & 0.000 & 0.915 & -5.721 & 5.001 & -0.504 & 0.498 & -0.252 & 7.127 & 0.000 & 0.000 & 0.001 & 0.000 & 0.000 & 0.000 & 1880.95 \\
\hline FMER & -0.005 & 0.058 & 1.765 & -11.931 & 9.523 & -0.951 & 0.999 & -0.422 & 8.063 & 2.032 & 0.284 & 0.080 & 0.000 & 0.000 & 0.000 & 1855.60 \\
\hline ISBC & 0.070 & 0.056 & 1.326 & -8.360 & 7.474 & -0.570 & 0.734 & 0.004 & 7.640 & 0.000 & 0.000 & 0.000 & 0.000 & 0.000 & 0.000 & 1645.87 \\
\hline
\end{tabular}

Table B.10: Descriptive statistics of the daily returns generated by the 25 U.S. banks with the largest market value, as recorded at the beginning of 2011, in the period between January 3, 2011 and December 31, 2015. From left to right the table reports, for each stock, the mean (\%), the median $(\%)$, the standard deviation (\%), the minimum (\%), the maximum (\%), the first and the third quartiles (\%), the skewness, the kurtosis, the p-value of the Ljung-Box test (\%), at the significance level of the $5 \%$, applied for both the returns $(L B)$ and the squared returns $(S L B)$ with lags equal to 1, 5 and 10, respectively, and the market value recorded in January 2011. 


\begin{tabular}{|c|c|c|c|c|c|c|c|c|c|c|c|c|c|c|c|c|}
\hline STOCK & MEAN & MED & ST DEV & MIN & MAX & $1^{s t} \mathrm{Q}$ & $3^{r d} \mathrm{Q}$ & SKEW & KURT & $L B_{1}$ & $L B_{5}$ & $L B_{10}$ & $S L B_{1}$ & $S L B_{5}$ & $S L B_{10}$ & MV \\
\hline $\mathrm{AIG}$ & -0.500 & -0.055 & 6.469 & -93.626 & 35.853 & -0.853 & 0.681 & -5.387 & 75.716 & 0.000 & 0.000 & 0.000 & 0.000 & 0.000 & 0.000 & 164653.50 \\
\hline BRKA & 0.011 & -0.001 & 1.717 & -12.883 & 14.953 & -0.523 & 0.536 & 0.622 & 24.924 & 93.137 & 0.610 & 0.965 & 0.000 & 0.000 & 0.000 & 110164.70 \\
\hline MET & -0.045 & 0.000 & 3.687 & -31.156 & 24.686 & -0.991 & 0.915 & -0.396 & 23.343 & 40.365 & 1.162 & 3.180 & 0.000 & 0.000 & 0.000 & 37890.41 \\
\hline PRU & -0.117 & -0.055 & 3.973 & -26.327 & 32.390 & -0.989 & 0.964 & 0.207 & 22.244 & 20.640 & 0.081 & 0.203 & 0.000 & 0.000 & 0.000 & 36860.64 \\
\hline ALL & -0.066 & 0.000 & 2.761 & -23.799 & 19.628 & -0.735 & 0.621 & -0.579 & 28.043 & 93.526 & 0.017 & 0.000 & 0.000 & 0.000 & 0.000 & 32610.33 \\
\hline TRV & 0.002 & 0.000 & 2.655 & -20.067 & 22.758 & -0.917 & 0.850 & 0.438 & 20.729 & 0.000 & 0.000 & 0.000 & 0.000 & 0.000 & 0.000 & 28563.55 \\
\hline HIG & -0.219 & -0.034 & 6.060 & -72.486 & 70.487 & -1.020 & 0.935 & -0.386 & 64.131 & 15.143 & 0.000 & 0.000 & 36.736 & 0.000 & 0.000 & 24387.18 \\
\hline $\mathrm{AFL}$ & -0.002 & 0.021 & 2.601 & -18.902 & 14.961 & -0.801 & 0.700 & -0.530 & 18.381 & 0.100 & 0.001 & 0.000 & 0.000 & 0.000 & 0.000 & 22472.57 \\
\hline PGR & -0.090 & -0.082 & 2.382 & -13.947 & 21.490 & -1.010 & 0.888 & 0.536 & 17.712 & 6.330 & 0.000 & 0.000 & 0.006 & 0.000 & 0.000 & 20556.40 \\
\hline $\mathrm{L}$ & -0.015 & 0.091 & 2.745 & -19.939 & 21.220 & -0.906 & 0.946 & -0.820 & 20.944 & 0.010 & 0.001 & 0.011 & 0.000 & 0.000 & 0.000 & 18655.31 \\
\hline LNC & -0.137 & 0.018 & 4.884 & -50.891 & 36.235 & -0.981 & 0.931 & -1.672 & 39.635 & 3.661 & 0.073 & 0.093 & 0.000 & 0.000 & 0.000 & 16040.52 \\
\hline MMC & -0.036 & -0.038 & 2.033 & -13.075 & 11.690 & -0.924 & 0.829 & 0.078 & 10.357 & 0.000 & 0.000 & 0.000 & 0.000 & 0.000 & 0.000 & 15753.62 \\
\hline PFG & -0.098 & 0.000 & 4.252 & -31.978 & 34.190 & -0.938 & 1.053 & 0.098 & 20.411 & 61.640 & 0.000 & 0.001 & 0.000 & 0.000 & 0.000 & 13717.85 \\
\hline GNW & -0.332 & -0.088 & 7.528 & -78.552 & 63.599 & -1.295 & 0.939 & -0.014 & 44.676 & 0.255 & 0.000 & 0.000 & 0.000 & 0.000 & 0.000 & 13158.24 \\
\hline CNA & -0.091 & 0.000 & 3.326 & -39.476 & 24.106 & -1.058 & 0.935 & -2.559 & 39.779 & 97.177 & 5.119 & 0.584 & 0.000 & 0.000 & 0.000 & 7953.97 \\
\hline MBI & -0.357 & -0.087 & 6.493 & -41.264 & 38.219 & -1.786 & 1.198 & -0.076 & 12.808 & 4.703 & 0.584 & 5.430 & 0.000 & 0.000 & 0.000 & 7842.20 \\
\hline WRB & -0.003 & -0.095 & 2.284 & -11.607 & 15.049 & -1.062 & 0.903 & 0.931 & 12.003 & 0.007 & 0.003 & 0.008 & 0.000 & 0.000 & 0.000 & 7607.63 \\
\hline CINF & -0.057 & -0.022 & 2.653 & -22.399 & 16.765 & -0.836 & 0.729 & -0.559 & 18.647 & 0.001 & 0.000 & 0.000 & 0.000 & 0.000 & 0.000 & 7239.32 \\
\hline AIZ & -0.049 & 0.034 & 3.069 & -28.679 & 19.721 & -0.869 & 0.903 & -0.962 & 24.650 & 29.164 & 0.012 & 0.000 & 0.000 & 0.000 & 0.000 & 6239.33 \\
\hline TMK & -0.029 & 0.017 & 2.208 & -15.228 & 13.967 & -0.633 & 0.691 & -0.495 & 17.199 & 54.298 & 0.005 & 0.079 & 0.000 & 0.000 & 0.000 & 5853.66 \\
\hline UNM & -0.027 & -0.049 & 3.428 & -35.145 & 20.010 & -1.051 & 0.984 & -1.268 & 28.910 & 0.282 & 0.000 & 0.005 & 0.000 & 0.000 & 0.000 & 5751.81 \\
\hline ORI & -0.075 & 0.000 & 3.340 & -29.214 & 31.979 & -0.932 & 0.792 & 0.115 & 28.746 & 1.547 & 0.000 & 0.000 & 0.000 & 0.000 & 0.000 & 5033.62 \\
\hline $\mathrm{BRO}$ & -0.050 & 0.000 & 2.077 & -17.218 & 11.196 & -0.867 & 0.835 & -0.630 & 13.710 & 18.359 & 15.353 & 23.086 & 0.378 & 0.001 & 0.000 & 4632.19 \\
\hline CNO & -0.198 & 0.000 & 6.043 & -55.048 & 59.157 & -1.009 & 0.851 & -0.891 & 36.127 & 0.000 & 0.000 & 0.000 & 0.000 & 0.000 & 0.000 & 3741.03 \\
\hline KMPR & -0.138 & -0.045 & 2.827 & -16.981 & 20.799 & -1.066 & 0.871 & -0.261 & 16.077 & 36.300 & 0.347 & 0.002 & 0.000 & 0.000 & 0.000 & 3326.30 \\
\hline
\end{tabular}

Table B.11: Descriptive statistics of the daily returns generated by the 25 U.S. insurance companies with the largest market value, as recorded at the beginning of 2006, in the period between January 3, 2006 and December 31, 2008. From left to right the table reports, for each stock, the mean (\%), the median (\%), the standard deviation (\%), the minimum (\%), the maximum (\%), the first and the third quartiles $(\%)$, the skewness, the kurtosis, the p-value of the Ljung-Box test (\%), at the significance level of the $5 \%$, applied for both the returns $(L B)$ and the squared returns $(S L B)$ with lags equal to 1, 5 and 10, respectively, and the market value recorded in January 2006. 


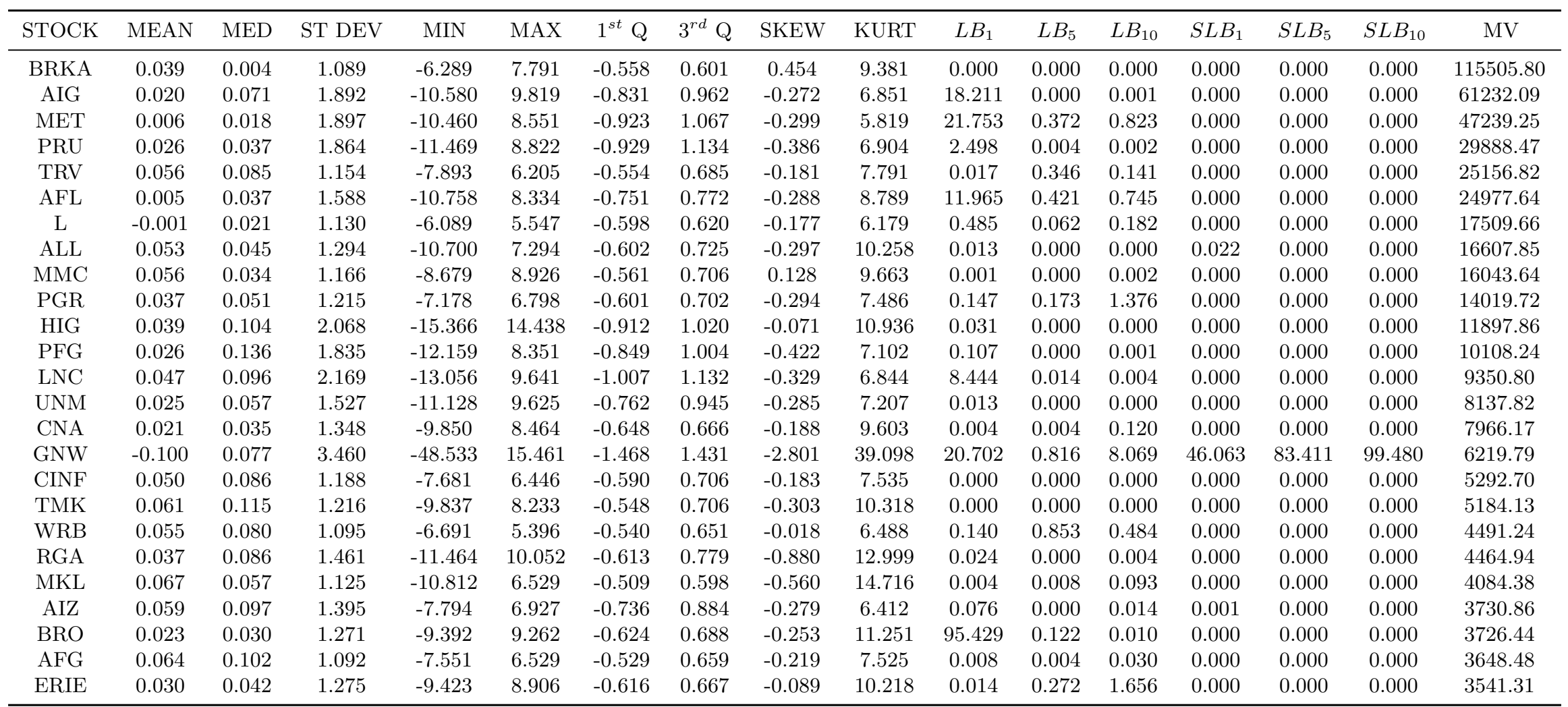

Table B.12: Descriptive statistics of the daily returns generated by the 25 U.S. insurance companies with the largest market value, as recorded at the beginning of 2011, in the period between January 3, 2011 and December 31, 2015. From left to right the table reports, for each stock, the mean (\%), the median (\%), the standard deviation (\%), the minimum (\%), the maximum (\%), the first and the third quartiles (\%), the skewness, the kurtosis, the p-value of the Ljung-Box test (\%), at the significance level of the $5 \%$, applied for both the returns $(L B)$ and the squared returns $(S L B)$ with lags equal to 1,5 and 10, respectively, and the market value recorded in January 2011. 


\begin{tabular}{|c|c|c|c|c|c|c|c|c|c|c|c|c|c|c|c|}
\hline POR & MEA & MED & STD & MIN & MAX & $1^{s t} \mathrm{Q}$ & $3^{r d} \mathrm{Q}$ & SKE & KUR & $L B_{1}$ & $L B_{5}$ & $L B_{10}$ & $S L B_{1}$ & $S L B_{5}$ & $S L B_{10}$ \\
\hline Agric & 0.096 & 0.050 & 2.701 & -15.270 & 18.240 & .930 & 1.140 & 0.078 & 10.597 & 35.606 & 0.424 & 0.016 & 0.268 & 0.000 & 0.000 \\
\hline Food & 0.010 & 0.030 & 1.165 & -7.250 & 7.400 & -0.485 & 0.580 & -0.076 & 12.281 & 1.713 & 0.000 & 0.000 & 0.000 & 0.000 & 0.000 \\
\hline Soda & -0.012 & 0.070 & 1.629 & -8.020 & 11.140 & -0.675 & 0.715 & 0.054 & 10.820 & 93.185 & 3.668 & 0.045 & 0.000 & 0.000 & 0.000 \\
\hline Beer & .031 & 0.040 & 1.187 & -7.700 & 10.120 & -0.480 & 0.530 & 0.811 & 18.398 & 0.018 & 0.000 & 0.000 & 0.000 & 0.000 & 0.000 \\
\hline Smoke & 0.030 & 0.070 & 1.479 & -7.400 & 13.310 & -0.600 & 0.725 & 0.338 & 16.033 & 12.642 & 0.000 & 0.000 & 0.665 & 0.000 & 0.000 \\
\hline Toys & -0.040 & 0.010 & 1.791 & -9.630 & 9.250 & -0.855 & 0.780 & -0.140 & 8.508 & 37.202 & 2.289 & 0.544 & 0.000 & 0.000 & 0.000 \\
\hline Fun & -0.083 & 0.050 & 2.423 & -12.170 & 16.580 & -0.900 & 0.830 & 0.023 & 11.578 & 0.003 & 0.000 & 0.002 & 0.000 & 0.000 & 0.000 \\
\hline Books & -0.124 & -0.060 & 1.953 & -11.240 & 11.180 & -0.785 & 0.590 & 0.004 & 11.861 & 42.319 & 0.069 & 0.341 & 0.000 & 0.000 & 0.000 \\
\hline Hshld & 0.010 & 0.030 & 1.260 & -7.250 & 9.440 & -0.455 & 0.520 & 0.077 & 13.616 & 0.002 & 0.000 & 0.000 & 0.000 & 0.000 & 0.000 \\
\hline Clths & -0.023 & -0.010 & 2.012 & -11.520 & 12.690 & -0.905 & 0.895 & 0.099 & 8.974 & 48.150 & 0.212 & 0.680 & 0.000 & 0.000 & 0.000 \\
\hline Hlth & -0.038 & 0.020 & 1.348 & -9.020 & 8.290 & -0.610 & 0.545 & -1.041 & 15.622 & 19.523 & 0.002 & 0.000 & 0.000 & 0.000 & 0.000 \\
\hline MedEq & -0.026 & 0.030 & 1.349 & -7.210 & 11.690 & -0.560 & 0.560 & 0.153 & 15.334 & 55.714 & 0.057 & 0.319 & 0.000 & 0.000 & 0.000 \\
\hline Drugs & 0.004 & 0.020 & 1.258 & -6.530 & 11.140 & -0.485 & 0.580 & 0.459 & 16.120 & 0.017 & 0.000 & 0.000 & 0.000 & 0.000 & 0.000 \\
\hline Chems & -0.010 & 0.110 & 2.039 & -11.380 & 13.060 & -0.720 & 0.880 & -0.416 & 11.100 & 4.333 & 0.002 & 0.010 & 0.000 & 0.000 & 0.000 \\
\hline Rubbr & -0.015 & 0.000 & 1.701 & -10.140 & 7.800 & -0.740 & 0.840 & -0.384 & 7.555 & 0.178 & 1.160 & 11.422 & 0.000 & 0.000 & 0.000 \\
\hline Txtls & -0.052 & -0.010 & 2.114 & -12.230 & 10.030 & -1.010 & 0.820 & -0.143 & 8.827 & 14.373 & 2.701 & 0.045 & 0.002 & 0.000 & 0.000 \\
\hline BldMt & -0.050 & 0.020 & 1.889 & -10.670 & 8.540 & -0.835 & 0.795 & -0.365 & 8.230 & 61.661 & 2.884 & 4.807 & 0.000 & 0.000 & 0.000 \\
\hline Cnstr & -0.057 & -0.050 & 2.767 & -12.400 & 15.560 & -1.355 & 1.280 & 0.091 & 7.184 & 28.169 & 3.002 & 9.516 & 0.000 & 0.000 & 0.000 \\
\hline Steel & 0.011 & 0.160 & 3.015 & -15.930 & 20.060 & -1.190 & 1.430 & -0.033 & 10.453 & 54.236 & 0.540 & 0.193 & 0.000 & 0.000 & 0.000 \\
\hline FabPr & -0.023 & 0.100 & 2.126 & -11.830 & 10.520 & -0.965 & 0.900 & -0.702 & 8.651 & 15.807 & 0.124 & 0.381 & 0.000 & 0.000 & 0.000 \\
\hline Mach & -0.016 & 0.150 & 2.144 & -12.250 & 13.910 & -0.895 & 1.025 & -0.315 & 11.089 & 18.366 & 0.000 & 0.000 & 0.000 & 0.000 & 0.000 \\
\hline ElcEq & 0.000 & 0.100 & 2.006 & -13.100 & 14.080 & -0.740 & 0.875 & -0.039 & 12.589 & 0.492 & 0.000 & 0.000 & 0.000 & 0.000 & 0.000 \\
\hline Autos & -0.080 & 0.070 & 2.349 & -11.230 & 11.700 & -0.995 & 1.010 & -0.062 & 8.630 & 99.063 & 1.838 & 3.530 & 0.000 & 0.000 & 0.000 \\
\hline Aero & -0.009 & 0.070 & 1.777 & -7.650 & 13.570 & -0.725 & 0.805 & 0.497 & 12.728 & 2.192 & 0.000 & 0.000 & 0.000 & 0.000 & 0.000 \\
\hline Ships & 0.004 & 0.030 & 1.715 & -9.400 & 10.620 & -0.810 & 0.815 & -0.110 & 9.046 & 0.011 & 0.001 & 0.001 & 0.000 & 0.000 & 0.000 \\
\hline Guns & 0.050 & 0.040 & 1.669 & -9.000 & 10.220 & -0.670 & 0.855 & -0.316 & 9.248 & 0.000 & 0.000 & 0.000 & 0.000 & 0.000 & 0.000 \\
\hline Gold & 0.017 & -0.050 & 3.120 & -14.160 & 25.560 & -1.505 & 1.530 & 1.087 & 14.187 & 3.891 & 0.245 & 0.399 & 0.000 & 0.000 & 0.000 \\
\hline Mines & 0.059 & 0.210 & 3.299 & -16.990 & 19.850 & -1.425 & 1.690 & 0.090 & 8.463 & 63.781 & 4.439 & 19.951 & 0.000 & 0.000 & 0.000 \\
\hline Coal & 0.014 & 0.000 & 4.170 & -19.340 & 21.360 & -1.805 & 1.885 & -0.204 & 7.777 & 37.757 & 0.120 & 0.013 & 0.000 & 0.000 & 0.000 \\
\hline Oil & 0.039 & 0.120 & 2.417 & -15.380 & 19.270 & -1.030 & 1.230 & 0.181 & 14.674 & 0.004 & 0.000 & 0.000 & 0.000 & 0.000 & 0.000 \\
\hline Util & 0.016 & 0.100 & 1.608 & -8.920 & 14.430 & -0.545 & 0.660 & 0.934 & 18.802 & 0.001 & 0.000 & 0.000 & 0.000 & 0.000 & 0.000 \\
\hline Telcm & -0.001 & 0.070 & 1.748 & -9.670 & 14.510 & -0.645 & 0.665 & 0.698 & 17.768 & 3.763 & 0.000 & 0.000 & 0.007 & 0.000 & 0.000 \\
\hline PerSv & -0.004 & 0.000 & 1.749 & -8.690 & 8.950 & -0.740 & 0.795 & -0.139 & 7.585 & 9.509 & 0.008 & 0.003 & 0.000 & 0.000 & 0.000 \\
\hline BusSv & -0.022 & 0.040 & 1.616 & -7.890 & 11.940 & -0.670 & 0.660 & 0.301 & 11.959 & 0.398 & 0.000 & 0.000 & 0.000 & 0.000 & 0.000 \\
\hline Comps & -0.011 & 0.040 & 1.830 & -9.890 & 11.870 & -0.830 & 0.795 & 0.166 & 9.166 & 0.426 & 0.011 & 0.110 & 0.000 & 0.000 & 0.000 \\
\hline Chips & -0.051 & 0.020 & 1.841 & -8.900 & 10.700 & -0.905 & 0.860 & 0.002 & 8.591 & 0.486 & 0.001 & 0.004 & 0.000 & 0.000 & 0.000 \\
\hline LabEq & -0.014 & 0.070 & 1.655 & -9.000 & 12.700 & -0.700 & 0.760 & -0.023 & 11.949 & 60.608 & 0.006 & 0.020 & 0.000 & 0.000 & 0.000 \\
\hline Paper & -0.043 & 0.050 & 1.535 & -9.610 & 8.630 & -0.610 & 0.630 & -0.275 & 10.620 & 0.123 & 0.000 & 0.000 & 0.002 & 0.000 & 0.000 \\
\hline
\end{tabular}




\begin{tabular}{|c|c|c|c|c|c|c|c|c|c|c|c|c|c|c|c|}
\hline Boxes & 0.028 & 0.110 & 1.881 & -9.040 & 10.920 & -0.855 & 0.800 & -0.016 & 7.738 & 78.496 & 4.496 & 8.536 & 0.000 & 0.000 & 0.000 \\
\hline Trans & -0.006 & 0.020 & 1.785 & -8.760 & 9.330 & -0.870 & 0.890 & -0.229 & 7.122 & 5.101 & 0.082 & 0.289 & 0.003 & 0.000 & 0.000 \\
\hline Whlsl & -0.028 & 0.030 & 1.476 & -8.500 & 9.740 & -0.585 & 0.650 & -0.210 & 11.386 & 6.327 & 0.135 & 0.228 & 0.000 & 0.000 & 0.000 \\
\hline Rtail & -0.016 & -0.040 & 1.612 & -8.310 & 11.750 & -0.745 & 0.710 & 0.375 & 9.746 & 7.334 & 0.006 & 0.011 & 0.000 & 0.000 & 0.000 \\
\hline Meals & 0.015 & 0.060 & 1.580 & -8.290 & 8.910 & -0.805 & 0.800 & 0.048 & 7.214 & 92.449 & 0.753 & 0.790 & 0.000 & 0.000 & 0.000 \\
\hline Banks & -0.060 & -0.040 & 2.735 & -16.280 & 16.750 & -0.860 & 0.665 & 0.314 & 12.215 & 1.838 & 0.093 & 0.102 & 0.000 & 0.000 & 0.000 \\
\hline Insur & -0.062 & 0.000 & 2.124 & -11.530 & 17.840 & -0.650 & 0.600 & 0.362 & 16.953 & 18.539 & 0.000 & 0.001 & 0.000 & 0.000 & 0.000 \\
\hline RlEst & -0.119 & -0.080 & 2.740 & -15.500 & 18.650 & -1.180 & 1.065 & 0.125 & 10.400 & 45.850 & 0.253 & 1.581 & 0.000 & 0.000 & 0.000 \\
\hline Fin & -0.046 & 0.030 & 2.824 & -16.270 & 17.940 & -1.035 & 1.045 & 0.232 & 11.047 & 33.562 & 0.005 & 0.046 & 0.000 & 0.000 & 0.000 \\
\hline Other & -0.033 & 0.020 & 1.570 & -9.880 & 9.990 & -0.540 & 0.550 & -0.270 & 12.982 & 44.794 & 1.394 & 0.069 & 0.000 & 0.000 & 0.000 \\
\hline
\end{tabular}

Table B.13: Descriptive statistics of the daily returns generated by the 48 industry portfolios in the period between January 3 , 2006 and December 31, 2008. From left to right the table reports, for each stock, the mean (\%), the median (\%), the standard deviation (\%), the minimum (\%), the maximum (\%), the first and the third quartiles (\%), the skewness, the kurtosis, and the p-value of the Ljung-Box test (\%), at the significance level of the $5 \%$, applied for both the returns $(L B)$ and the squared returns $(S L B)$ with lags equal to 1, 5 and 10 , respectively. 


\begin{tabular}{|c|c|c|c|c|c|c|c|c|c|c|c|c|c|c|c|}
\hline POR & MEA & MED & STD & MIN & MAX & $1^{s t} \mathrm{Q}$ & $3^{r d} \mathrm{Q}$ & SKE & KUR & $L B_{1}$ & $L B_{5}$ & $L B_{10}$ & $S L B_{1}$ & $S L B_{5}$ & $S L B_{10}$ \\
\hline Agric & 0.045 & 0.050 & 1.399 & -6.940 & 7.650 & -0.700 & 0.840 & 0.175 & 6.106 & 74.914 & 11.260 & 12.269 & 0.000 & 0.000 & 0.000 \\
\hline Food & 0.063 & 0.100 & 0.855 & -4.620 & 3.670 & -0.420 & 0.550 & -0.274 & 5.070 & 8.731 & 0.316 & 0.581 & 0.000 & 0.000 & 0.000 \\
\hline Soda & 0.062 & 0.060 & 1.066 & -6.570 & 5.150 & -0.490 & 0.650 & -0.202 & 6.850 & 0.007 & 0.000 & 0.000 & 0.000 & 0.000 & 0.000 \\
\hline Beer & 0.064 & 0.075 & 0.833 & -4.280 & 4.020 & -0.430 & 0.520 & -0.139 & 4.934 & 14.505 & 0.010 & 0.023 & 0.000 & 0.000 & 0.000 \\
\hline Smoke & 0.075 & 0.090 & 0.966 & -4.780 & 4.390 & -0.488 & 0.640 & -0.165 & 4.698 & 35.988 & 17.203 & 21.629 & 0.000 & 0.000 & 0.000 \\
\hline Toys & 0.043 & 0.070 & 1.300 & -8.290 & 6.680 & -0.600 & 0.790 & -0.270 & 6.759 & 34.771 & 50.545 & 67.397 & 0.000 & 0.000 & 0.000 \\
\hline Fun & 0.060 & 0.120 & 1.624 & -8.960 & 6.410 & -0.840 & 0.968 & -0.127 & 5.209 & 26.336 & 1.394 & 3.720 & 0.000 & 0.000 & 0.000 \\
\hline Books & 0.055 & 0.090 & 1.332 & -8.720 & 6.910 & -0.650 & 0.820 & -0.567 & 7.973 & 94.914 & 0.490 & 1.377 & 0.000 & 0.000 & 0.000 \\
\hline Hshld & 0.039 & 0.050 & 0.851 & -4.090 & 4.010 & -0.430 & 0.540 & -0.300 & 5.228 & 39.275 & 1.251 & 6.061 & 0.000 & 0.000 & 0.000 \\
\hline Clths & 0.066 & 0.070 & 1.332 & -8.330 & 6.790 & -0.610 & 0.818 & -0.286 & 6.471 & 81.731 & 0.000 & 0.000 & 0.000 & 0.000 & 0.000 \\
\hline Hlth & 0.058 & 0.130 & 1.264 & -10.100 & 5.040 & -0.580 & 0.770 & -0.971 & 9.741 & 91.847 & 9.331 & 0.267 & 0.001 & 0.000 & 0.000 \\
\hline MedEq & 0.062 & 0.100 & 1.075 & -6.910 & 4.780 & -0.480 & 0.708 & -0.528 & 6.520 & 17.852 & 1.430 & 0.098 & 0.000 & 0.000 & 0.000 \\
\hline Drugs & 0.083 & 0.110 & 1.034 & -4.720 & 4.550 & -0.430 & 0.670 & -0.312 & 5.204 & 68.920 & 7.524 & 1.500 & 0.000 & 0.000 & 0.000 \\
\hline Chems & 0.044 & 0.080 & 1.299 & -8.210 & 6.620 & -0.600 & 0.748 & -0.383 & 7.219 & 40.159 & 0.001 & 0.001 & 0.000 & 0.000 & 0.000 \\
\hline Rubbr & 0.069 & 0.100 & 1.204 & -5.960 & 6.710 & -0.620 & 0.730 & -0.177 & 5.742 & 34.775 & 0.009 & 0.137 & 0.000 & 0.000 & 0.000 \\
\hline Txtls & 0.107 & 0.130 & 1.534 & -8.130 & 6.830 & -0.700 & 0.988 & -0.262 & 5.268 & 94.660 & 0.008 & 0.048 & 0.000 & 0.000 & 0.000 \\
\hline BldMt & 0.055 & 0.100 & 1.434 & -8.410 & 6.330 & -0.720 & 0.850 & -0.204 & 5.708 & 49.169 & 0.003 & 0.017 & 0.000 & 0.000 & 0.000 \\
\hline Cnstr & 0.039 & 0.080 & 1.646 & -10.030 & 6.020 & -0.860 & 0.978 & -0.398 & 5.522 & 98.631 & 0.011 & 0.139 & 0.000 & 0.000 & 0.000 \\
\hline Steel & -0.016 & 0.000 & 1.730 & -10.760 & 8.600 & -0.945 & 0.960 & -0.163 & 6.304 & 88.503 & 0.044 & 0.281 & 0.000 & 0.000 & 0.000 \\
\hline FabPr & -0.009 & 0.060 & 2.007 & -15.450 & 8.170 & -1.108 & 1.070 & -0.266 & 7.224 & 94.000 & 0.034 & 0.211 & 0.000 & 0.000 & 0.000 \\
\hline Mach & 0.022 & 0.060 & 1.392 & -8.790 & 6.790 & -0.690 & 0.750 & -0.169 & 6.720 & 42.870 & 0.001 & 0.003 & 0.000 & 0.000 & 0.000 \\
\hline ElcEq & 0.025 & 0.040 & 1.351 & -7.740 & 7.560 & -0.718 & 0.740 & -0.069 & 6.608 & 57.250 & 0.049 & 0.335 & 0.000 & 0.000 & 0.000 \\
\hline Autos & 0.034 & 0.080 & 1.456 & -8.530 & 6.380 & -0.660 & 0.850 & -0.374 & 6.056 & 12.214 & 0.070 & 0.295 & 0.000 & 0.000 & 0.000 \\
\hline Aero & 0.059 & 0.095 & 1.167 & -7.030 & 5.050 & -0.568 & 0.740 & -0.457 & 6.455 & 85.949 & 0.328 & 3.428 & 0.000 & 0.000 & 0.000 \\
\hline Ships & 0.091 & 0.195 & 1.733 & -8.900 & 7.180 & -0.880 & 1.070 & -0.173 & 5.049 & 25.610 & 2.883 & 11.182 & 0.000 & 0.000 & 0.000 \\
\hline Guns & 0.106 & 0.120 & 1.069 & -5.730 & 3.740 & -0.488 & 0.770 & -0.345 & 4.931 & 32.729 & 6.839 & 18.811 & 0.000 & 0.000 & 0.000 \\
\hline Gold & -0.079 & -0.150 & 2.375 & -11.760 & 8.760 & -1.480 & 1.298 & 0.074 & 4.495 & 33.125 & 70.504 & 68.383 & 0.586 & 0.000 & 0.000 \\
\hline Mines & -0.043 & -0.045 & 1.882 & -8.500 & 10.040 & -1.010 & 0.970 & 0.060 & 5.541 & 6.054 & 0.108 & 0.554 & 0.000 & 0.000 & 0.000 \\
\hline Coal & -0.206 & -0.135 & 2.886 & -18.440 & 18.080 & -1.795 & 1.288 & -0.012 & 7.057 & 20.566 & 0.015 & 0.176 & 0.001 & 0.000 & 0.000 \\
\hline Oil & 0.009 & 0.020 & 1.422 & -8.350 & 5.480 & -0.720 & 0.780 & -0.342 & 6.057 & 54.006 & 0.005 & 0.001 & 0.003 & 0.000 & 0.000 \\
\hline Util & 0.039 & 0.070 & 0.886 & -6.050 & 4.550 & -0.470 & 0.558 & -0.311 & 6.626 & 8.517 & 0.007 & 0.020 & 0.000 & 0.000 & 0.000 \\
\hline Telcm & 0.058 & 0.110 & 0.960 & -6.600 & 4.650 & -0.440 & 0.628 & -0.521 & 6.756 & 52.461 & 0.002 & 0.013 & 0.000 & 0.000 & 0.000 \\
\hline PerSv & 0.033 & 0.080 & 1.345 & -8.650 & 4.810 & -0.700 & 0.888 & -0.457 & 5.261 & 25.112 & 29.973 & 29.929 & 0.000 & 0.000 & 0.000 \\
\hline BusSv & 0.060 & 0.090 & 1.106 & -6.310 & 4.870 & -0.490 & 0.678 & -0.290 & 5.843 & 91.176 & 0.049 & 0.141 & 0.000 & 0.000 & 0.000 \\
\hline Comps & 0.027 & 0.100 & 1.251 & -6.260 & 5.500 & -0.660 & 0.770 & -0.190 & 5.481 & 74.150 & 10.162 & 7.555 & 0.001 & 0.000 & 0.000 \\
\hline Chips & 0.057 & 0.090 & 1.245 & -5.760 & 5.070 & -0.600 & 0.760 & -0.117 & 4.863 & 99.802 & 1.614 & 0.298 & 0.000 & 0.000 & 0.000 \\
\hline LabEq & 0.062 & 0.100 & 1.318 & -7.460 & 5.740 & -0.620 & 0.780 & -0.283 & 6.327 & 73.759 & 0.251 & 0.216 & 0.000 & 0.000 & 0.000 \\
\hline Paper & 0.056 & 0.100 & 1.084 & -6.330 & 5.170 & -0.460 & 0.630 & -0.356 & 6.220 & 5.275 & 0.090 & 0.533 & 0.000 & 0.000 & 0.000 \\
\hline
\end{tabular}




\begin{tabular}{|c|c|c|c|c|c|c|c|c|c|c|c|c|c|c|c|}
\hline Boxes & 0.045 & 0.090 & 1.218 & -8.280 & 7.420 & -0.570 & 0.720 & -0.340 & 6.851 & 11.874 & 0.035 & 0.526 & 0.000 & 0.000 & 0.000 \\
\hline Trans & 0.053 & 0.130 & 1.187 & -6.010 & 4.760 & -0.588 & 0.720 & -0.321 & 5.009 & 90.781 & 0.800 & 1.182 & 0.000 & 0.000 & 0.000 \\
\hline Whlsl & 0.057 & 0.090 & 1.039 & -6.710 & 5.440 & -0.470 & 0.650 & -0.239 & 6.954 & 42.801 & 0.029 & 0.089 & 0.000 & 0.000 & 0.000 \\
\hline Rtail & 0.067 & 0.095 & 0.936 & -5.990 & 4.790 & -0.428 & 0.620 & -0.369 & 6.227 & 11.008 & 0.213 & 0.118 & 0.000 & 0.000 & 0.000 \\
\hline Meals & 0.063 & 0.090 & 0.982 & -5.440 & 4.870 & -0.468 & 0.638 & -0.313 & 6.208 & 4.122 & 0.084 & 0.000 & 0.000 & 0.000 & 0.000 \\
\hline Banks & 0.055 & 0.070 & 1.384 & -10.670 & 7.950 & -0.638 & 0.770 & -0.316 & 9.177 & 0.001 & 0.000 & 0.000 & 0.000 & 0.000 & 0.000 \\
\hline Insur & 0.066 & 0.115 & 1.149 & -8.520 & 6.940 & -0.510 & 0.690 & -0.342 & 9.017 & 0.021 & 0.000 & 0.000 & 0.000 & 0.000 & 0.000 \\
\hline RlEst & 0.048 & 0.070 & 1.427 & -10.310 & 6.600 & -0.680 & 0.820 & -0.367 & 7.586 & 54.196 & 0.036 & 0.024 & 0.000 & 0.000 & 0.000 \\
\hline Fin & 0.044 & 0.100 & 1.421 & -8.930 & 8.900 & -0.668 & 0.790 & -0.207 & 7.838 & 0.456 & 0.000 & 0.000 & 0.000 & 0.000 & 0.000 \\
\hline Other & 0.049 & 0.050 & 1.070 & -6.750 & 5.890 & -0.510 & 0.618 & -0.078 & 7.505 & 0.098 & 0.000 & 0.000 & 0.000 & 0.000 & 0.000 \\
\hline
\end{tabular}

Table B.14: Descriptive statistics of the daily returns generated by the 48 industry portfolios in the period between January 3, 2011 and December 31, 2015. From left to right the table reports, for each stock, the mean (\%), the median (\%), the standard deviation (\%), the minimum (\%), the maximum (\%), the first and the third quartiles (\%), the skewness, the kurtosis, and the p-value of the Ljung-Box test (\%), at the significance level of the $5 \%$, applied for both the returns $(L B)$ and the squared returns $(S L B)$ with lags equal to 1,5 and 10 , respectively. 


\section{Figures of primitive causality networks}

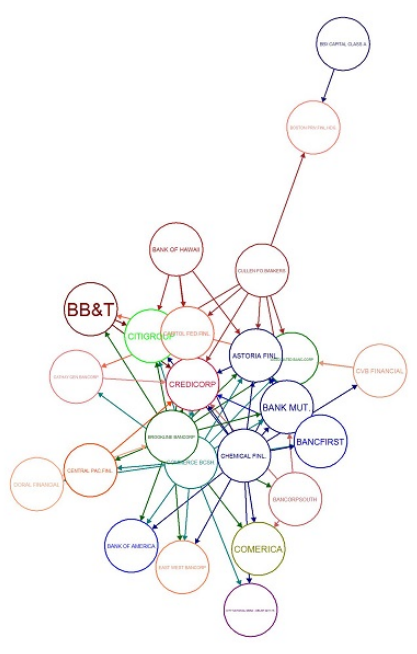

(a) Granger

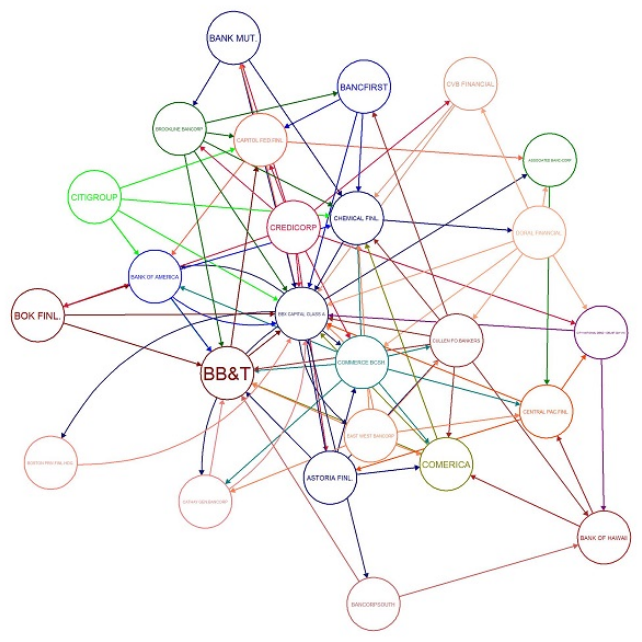

(c) $Q_{10}$ Quantile on Quantile

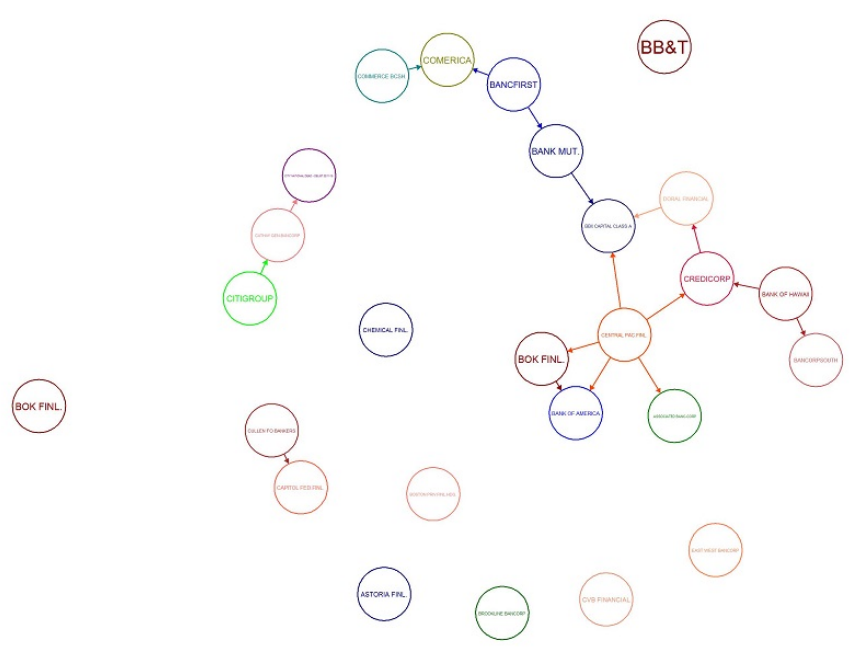

(b) $Q_{10}$ Base Quantile

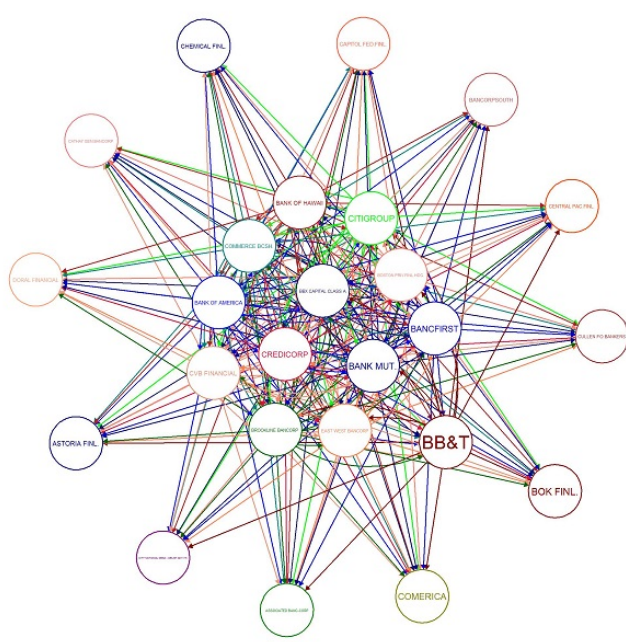

(d) $Q_{10}$ Not Parametric

Figure C.9: This figure visualizes 4 different networks for the period 2006-2008 relative to the first 25 banks ordered for market capitalization. In this case the networks visualized are some of the primitive networks used for the resulting network computations. Panel a) reports the network extracted by the standard granger causality. Panel b) indicates the network extracted by a quantile baseline regression methodology on the $10 \%$ quantile $q_{10}$. Panel c) indicates the network extracted by a quantile on quantile methodology on the $10 \%$ quantile $q_{10}$. Panel d) reports the network extracted by a not parametric methodologie on the $10 \%$ quantile $q_{10}$. All the causality regression are computed without the market factor. 


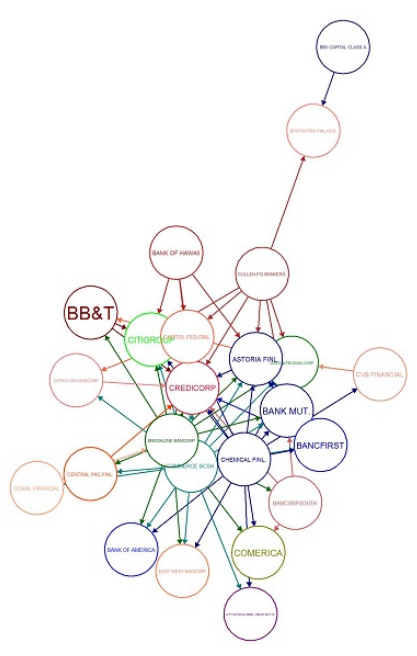

(a) Granger

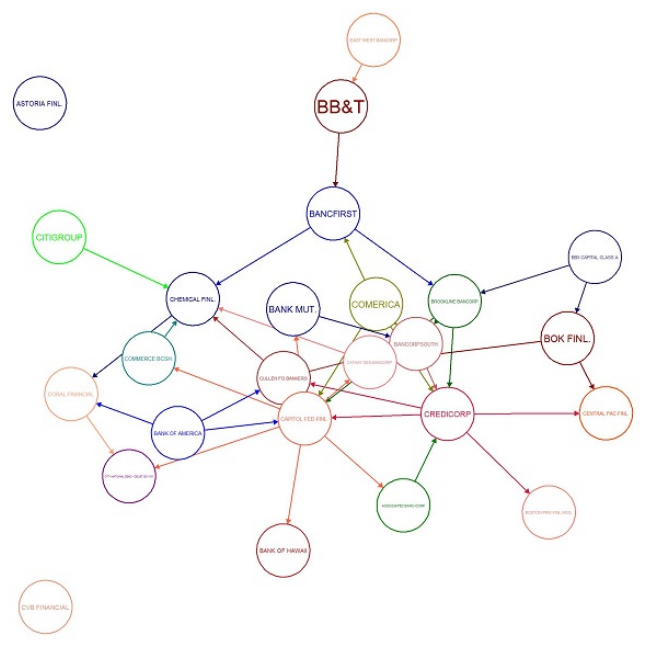

(c) $Q_{50}$ Quantile on Quantile

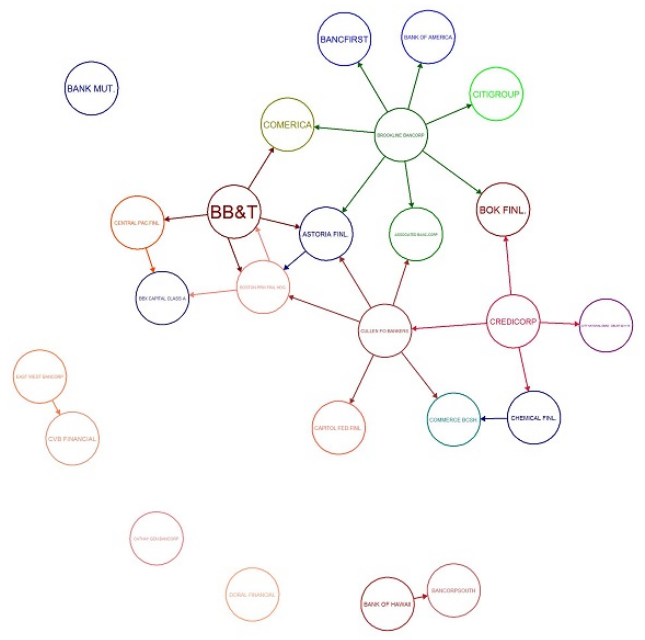

(b) $Q_{50}$ Base Quantile

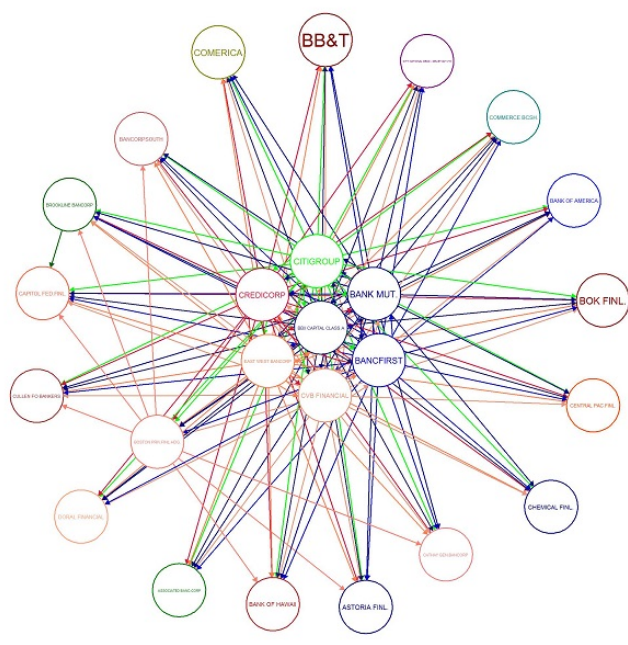

(d) $Q_{50}$ Not Parametric

Figure C.10: This figure visualizes 4 different networks for the period 2006-2008 relative to the first 25 banks ordered for market capitalization. In this case the networks visualized are some of the primitive networks used for the resulting network computations. Panel a) reports the network extracted by the standard granger causality. Panel b) indicates the network extracted by a quantile baseline regression methodology on the $50 \%$ quantile $q_{50}$. Panel c) indicates the network extracted by a quantile on quantile methodology on the $50 \%$ quantile $q_{50}$. Panel d) reports the network extracted by a not parametric methodology on the $50 \%$ quantile $q_{50}$. All the causality regression are computed without the market factor. 


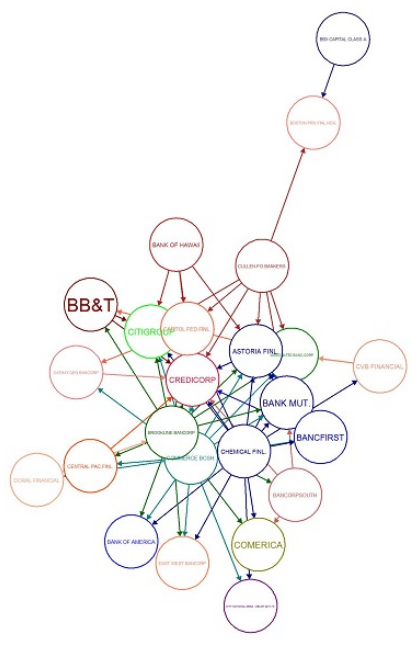

(a) Granger

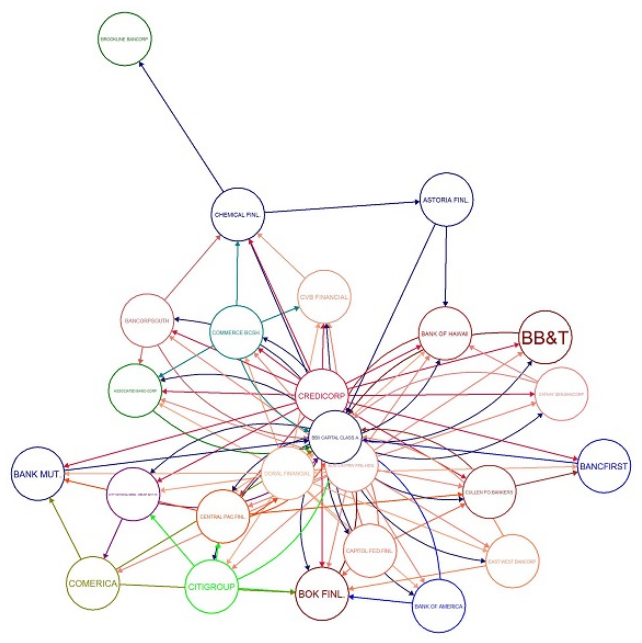

(c) $Q_{90}$ Quantile on Quantile

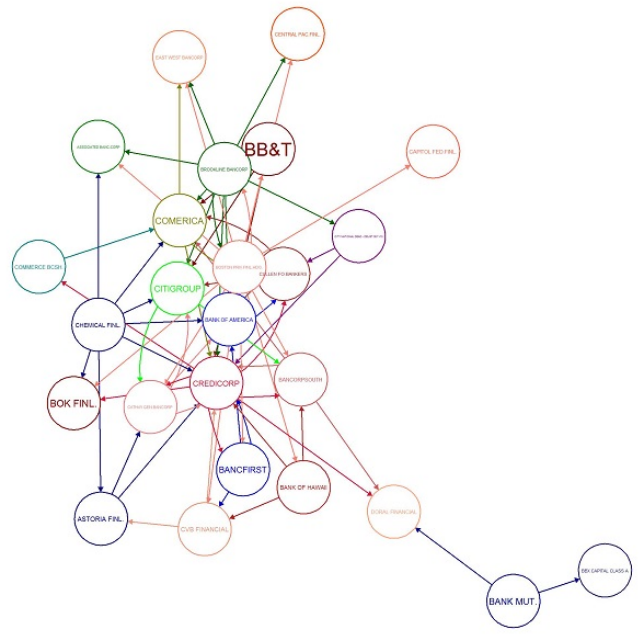

(b) $Q_{90}$ Base Quantile

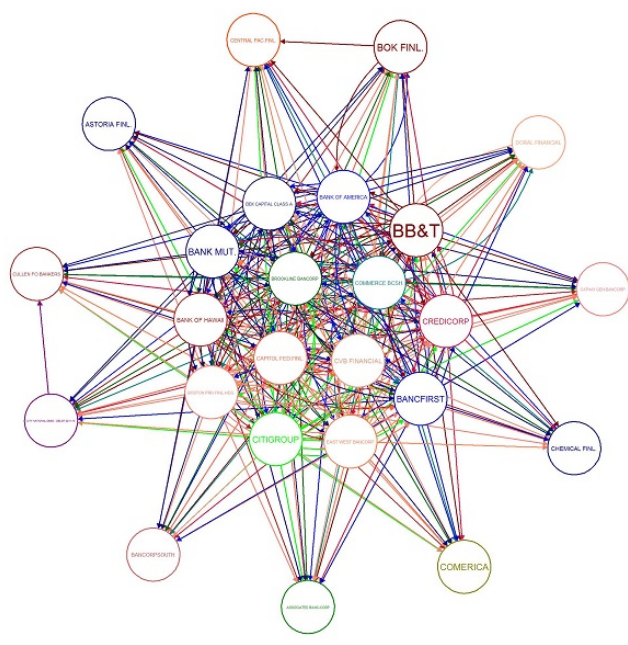

(d) $Q_{90}$ Not Parametric

Figure C.11: This figure visualizes 4 different networks for the period 2006-2008 relative to the first 25 banks ordered for market capitalization. In this case the networks visualized are some of the primitive networks used for the resulting network computations. Panel a) reports the network extracted by the standard granger causality. Panel b) indicates the network extracted by a quantile baseline regression methodology on the $90 \%$ quantile $q_{90}$. Panel c) indicates the network extracted by a quantile on quantile methodology on the $90 \%$ quantile $q_{90}$. Panel d) reports the network extracted by a not parametric methodology on the $90 \%$ quantile $q_{90}$. All the causality regression are computed without the market factor. 


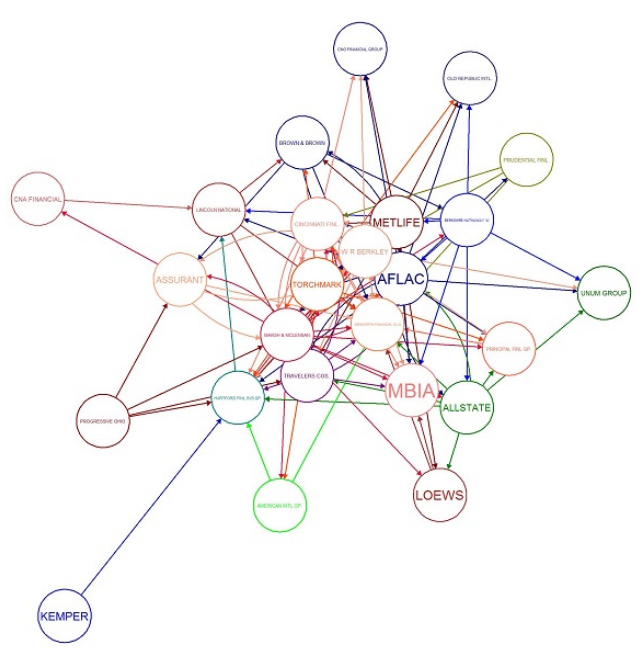

(a) Granger

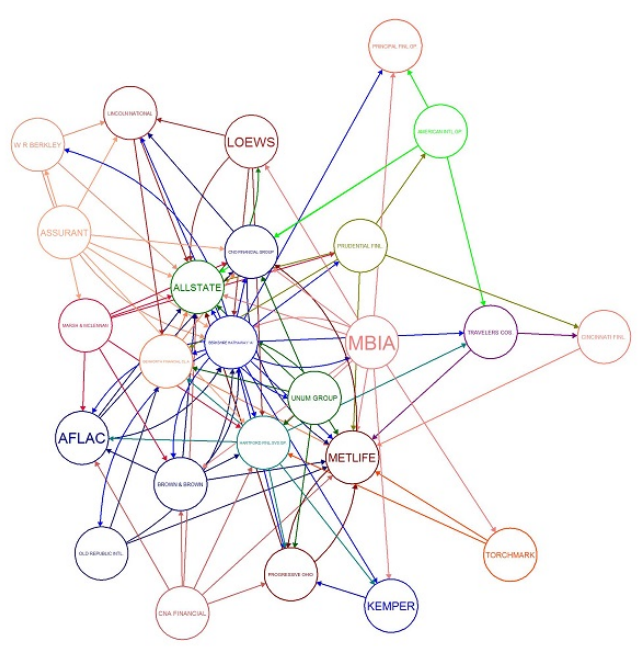

(c) $Q_{10}$ Quantile on Quantile

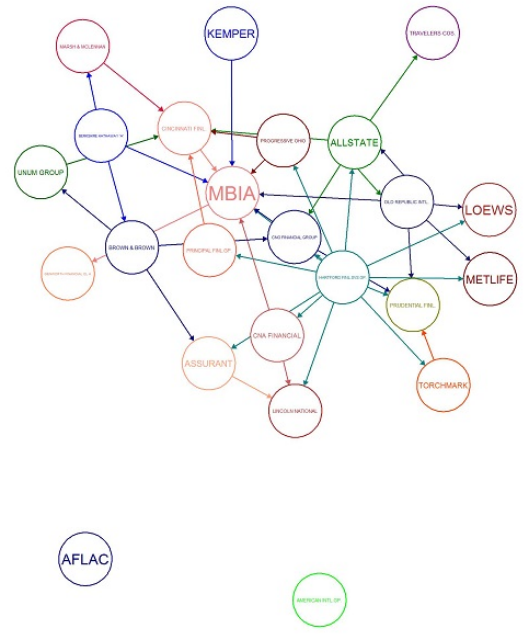

(b) $Q_{10}$ Base Quantile

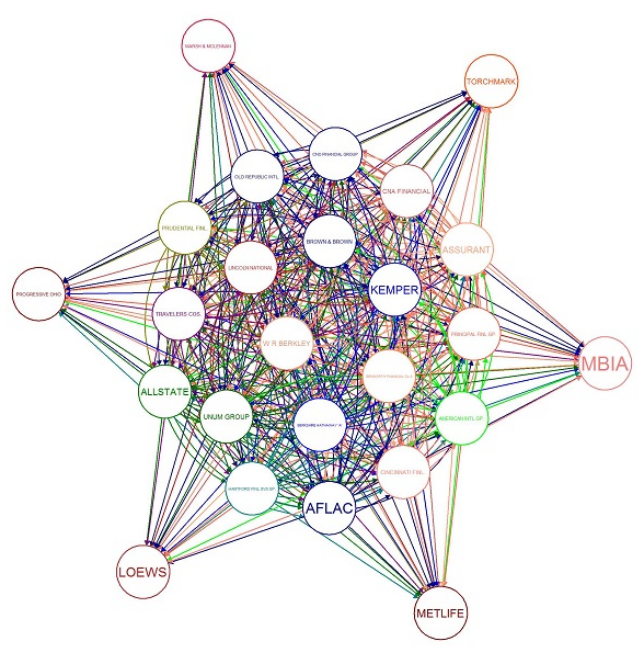

(d) $Q_{10}$ Not Parametric

Figure C.12: This figure visualizes 4 different networks for the period 2006-2008 relative to the first 25 Insurers ordered for market capitalization. In this case the networks visualized are some of the primitive networks used for the resulting network computations. Panel a) reports the network extracted by the standard granger causality. Panel b) indicates the network extracted by a quantile baseline regression methodology on the $10 \%$ quantile $q_{10}$. Panel c) indicates the network extracted by a quantile on quantile methodology on the $10 \%$ quantile $q_{10}$. Panel d) reports the network extracted by a not parametric methodologie on the $10 \%$ quantile $q_{10}$. All the causality regression are computed without the market factor. 


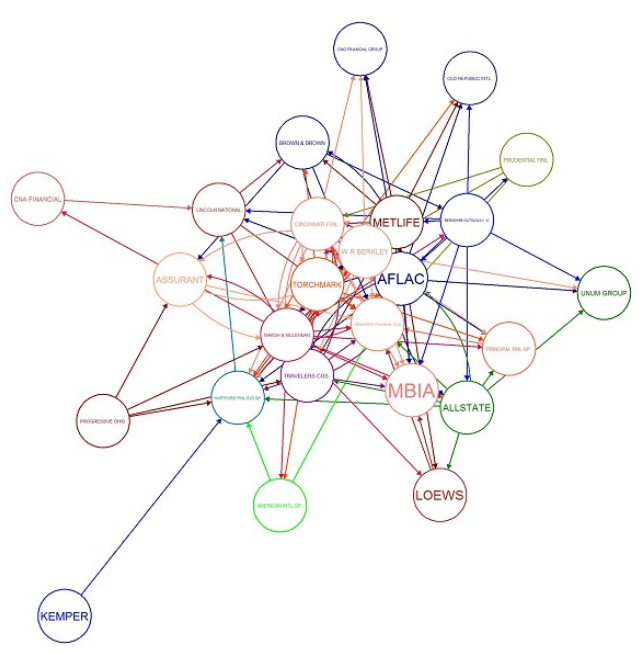

(a) Granger

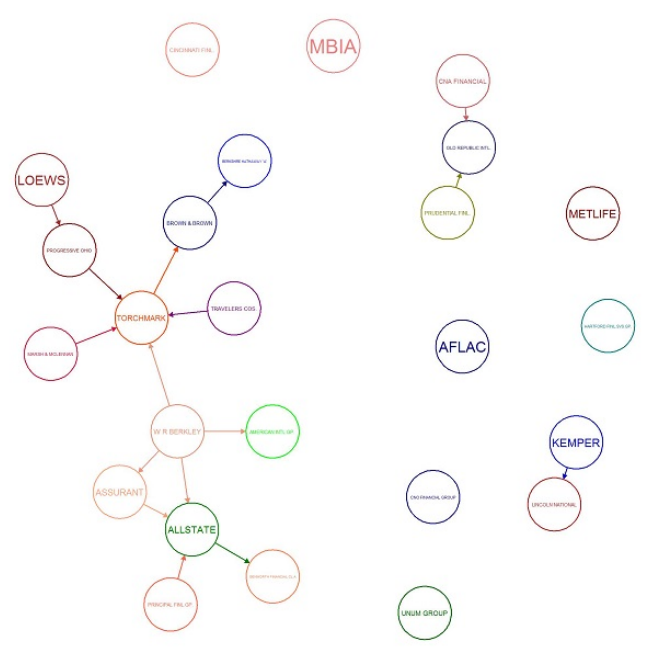

(c) $Q_{50}$ Quantile on Quantile

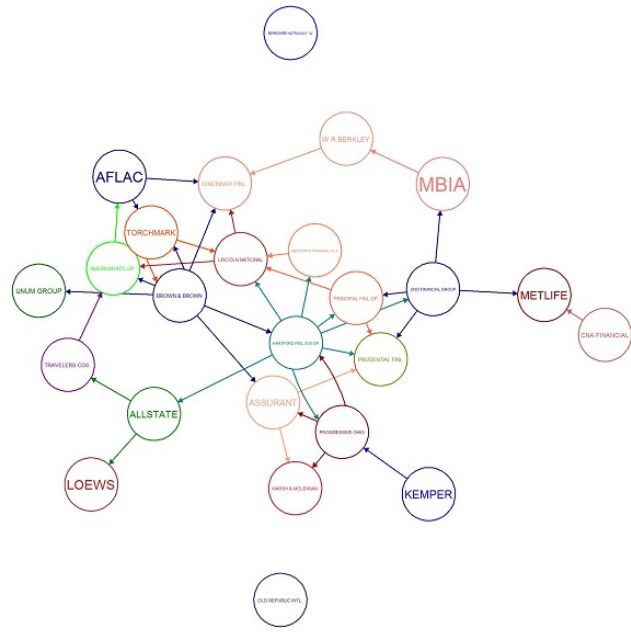

(b) $Q_{50}$ Base Quantile

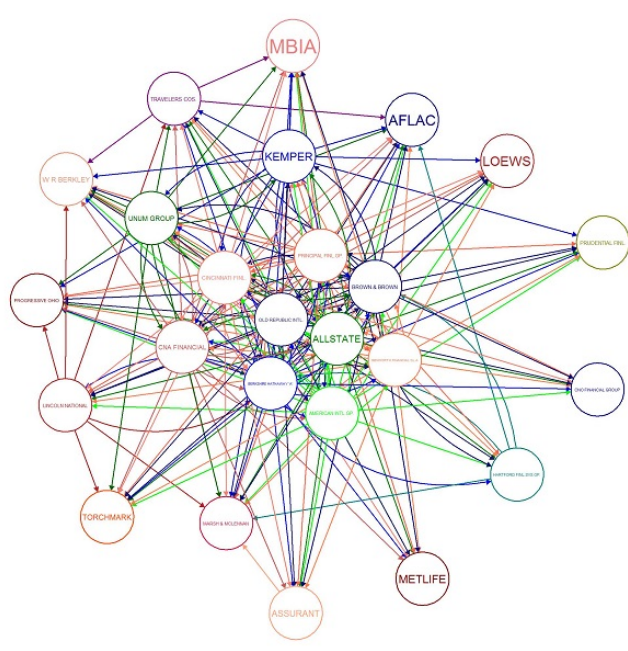

(d) $Q_{50}$ Not Parametric

Figure C.13: This figure visualizes 4 different networks for the period 2006-2008 relative to the first 25 Insurers ordered for market capitalization. In this case the networks visualized are some of the primitive networks used for the resulting network computations. Panel a) reports the network extracted by the standard granger causality. Panel b) indicates the network extracted by a quantile baseline regression methodology on the $50 \%$ quantile $q_{50}$. Panel c) indicates the network extracted by a quantile on quantile methodology on the $50 \%$ quantile $q_{50}$. Panel d) reports the network extracted by a not parametric methodology on the $50 \%$ quantile $q_{50}$. All the causality regression are computed without the market factor. 


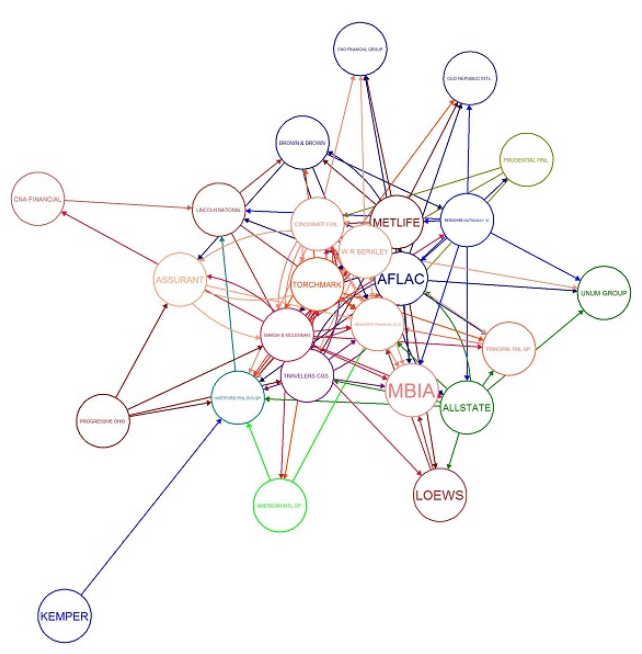

(a) Granger

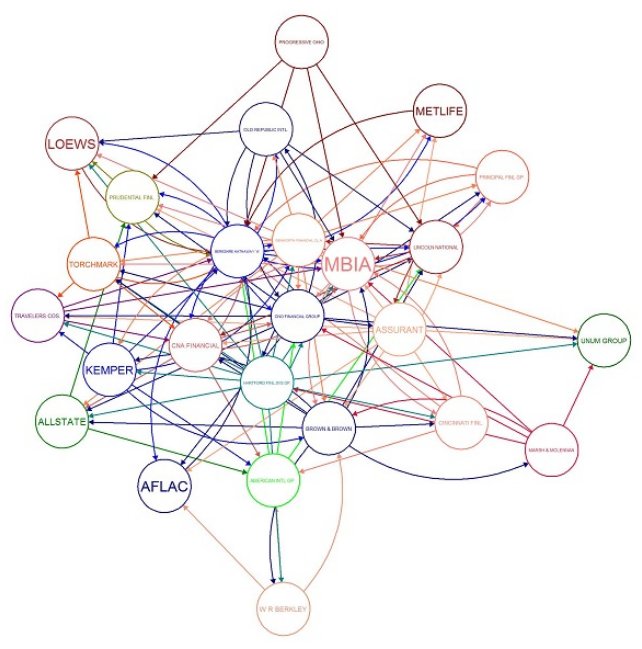

(c) $Q_{90}$ Quantile on Quantile

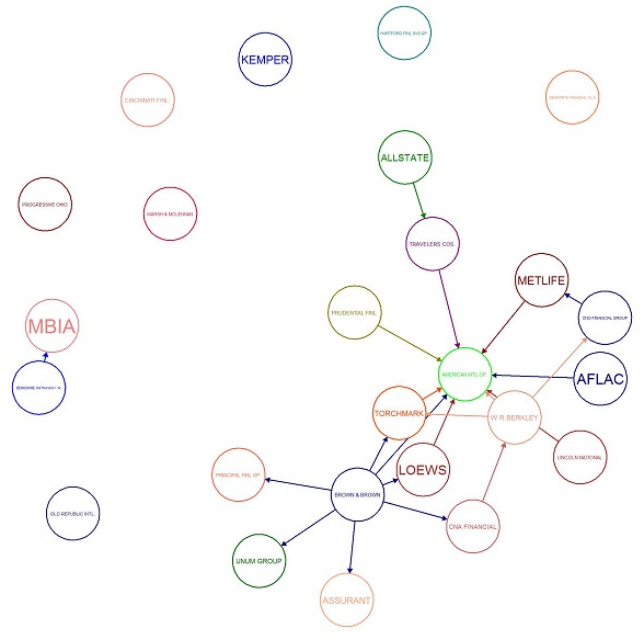

(b) $Q_{90}$ Base Quantile

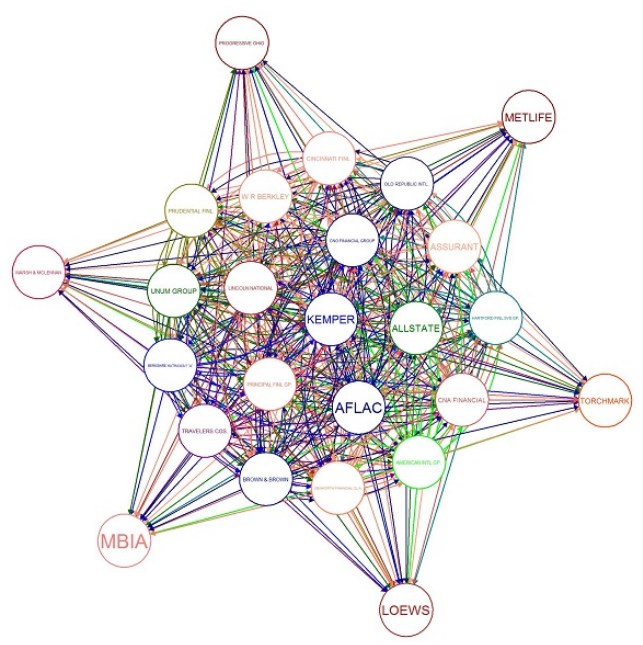

(d) $Q_{90}$ Not Parametric

Figure C.14: This figure visualizes 4 different networks for the period 2006-2008 relative to the first 25 Insurers ordered for market capitalization. In this case the networks visualized are some of the primitive networks used for the resulting network computations. Panel a) reports the network extracted by the standard granger causality. Panel b) indicates the network extracted by a quantile baseline regression methodology on the $90 \%$ quantile $q_{90}$. Panel c) indicates the network extracted by a quantile on quantile methodology on the $90 \%$ quantile $q_{90}$. Panel d) reports the network extracted by a not parametric methodology on the $90 \%$ quantile $q_{90}$. All the causality regression are computed without the market factor. 


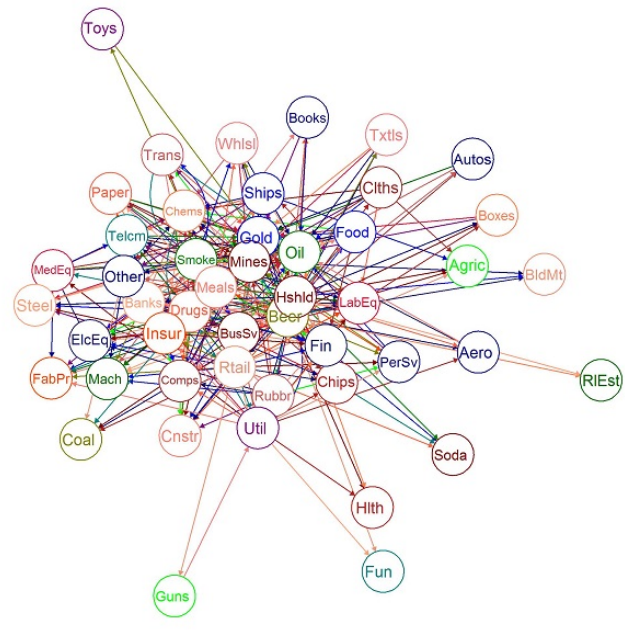

(a) Granger

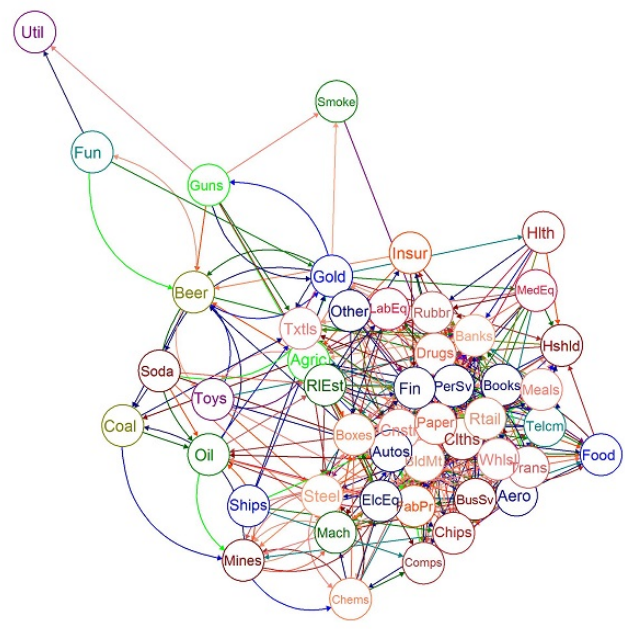

(c) $Q_{10}$ Quantile on Quantile

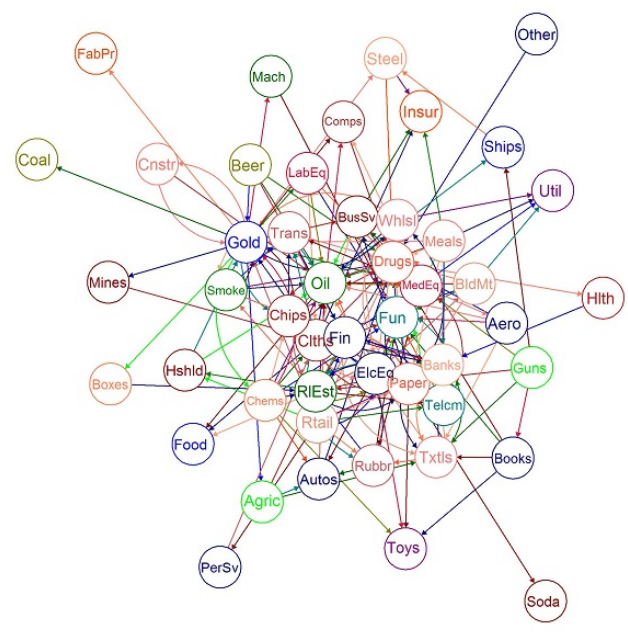

(b) $Q_{10}$ Base Quantile

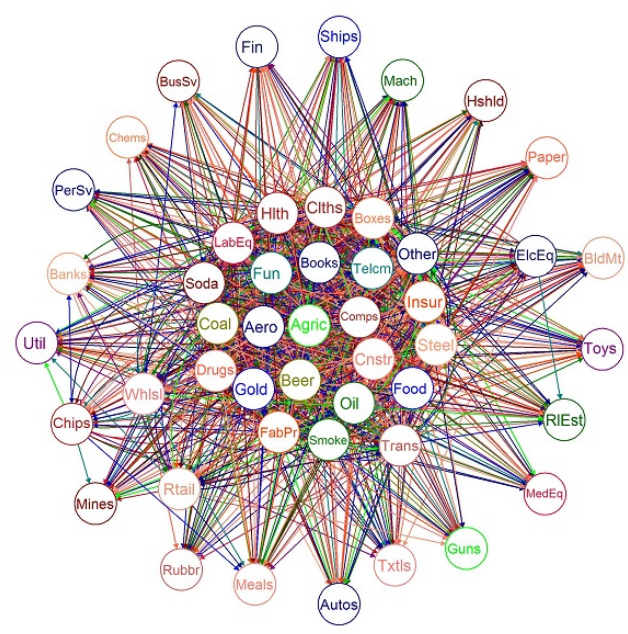

(d) $Q_{10}$ Not Parametric

Figure C.15: This figure visualizes 4 different networks for the period 2006-2008 relative to the 48 Fama and French industry portofolios. In this case the networks visualized are some of the primitive networks used for the resulting network computations. Panel a) reports the network extracted by the standard granger causality. Panel b) indicates the network extracted by a quantile baseline regression methodology on the $10 \%$ quantile $q_{10}$. Panel c) indicates the network extracted by a quantile on quantile methodology on the $10 \%$ quantile $q_{10}$. Panel d) reports the network extracted by a not parametric methodologie on the $10 \%$ quantile $q_{10}$. All the causality regression are computed without the market factor. 


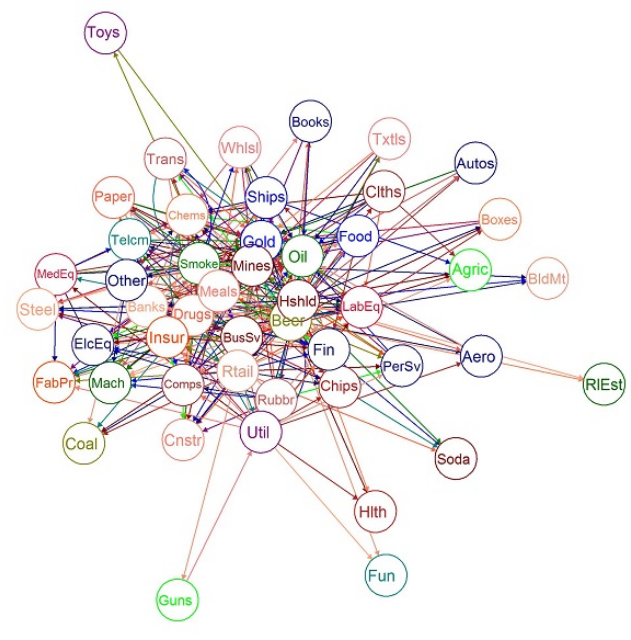

(a) Granger

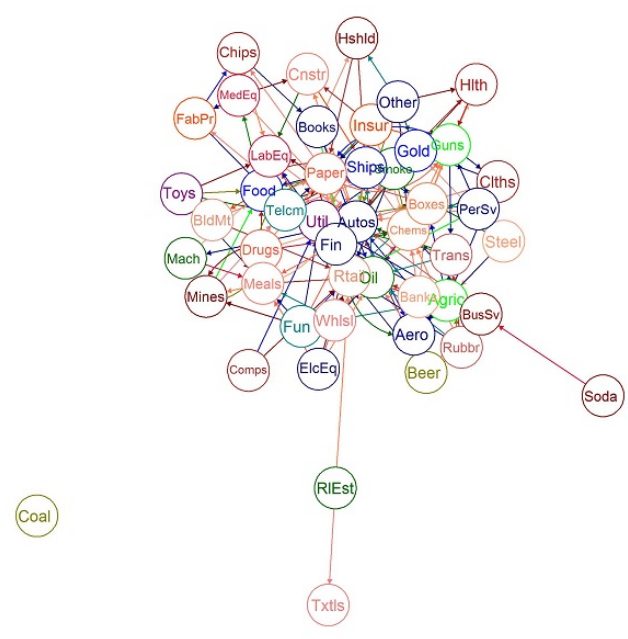

(c) $Q_{50}$ Quantile on Quantile

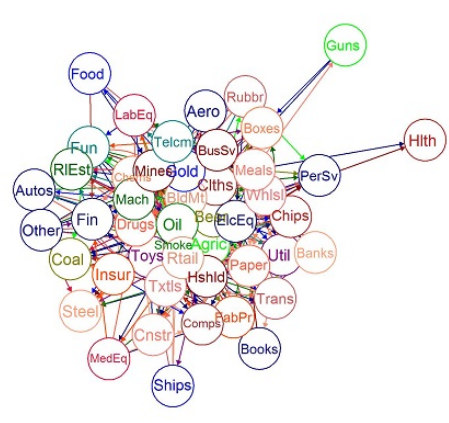

(b) $Q_{50}$ Base Quantile

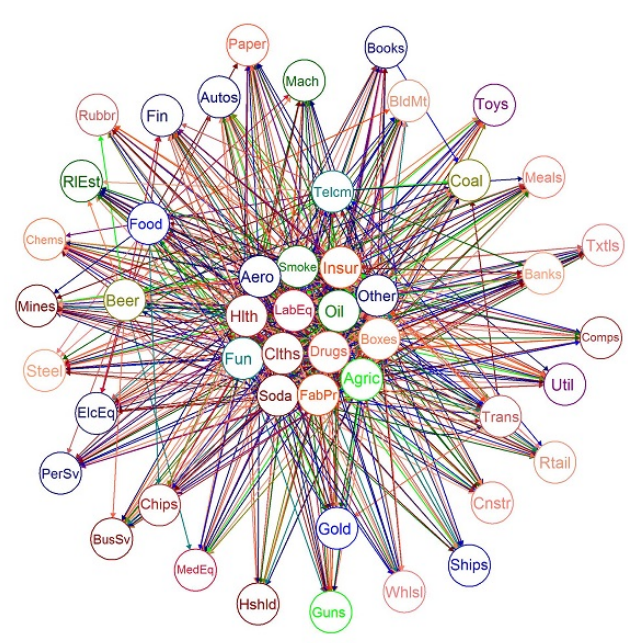

(d) $Q_{50}$ Not Parametric

Figure C.16: This figure visualizes 4 different networks for the period 2006-2008 relative to the 48 Fama and French industry portofolios. In this case the networks visualized are some of the primitive networks used for the resulting network computations. Panel a) reports the network extracted by the standard granger causality. Panel b) indicates the network extracted by a quantile baseline regression methodology on the $50 \%$ quantile $q_{50}$. Panel c) indicates the network extracted by a quantile on quantile methodology on the $50 \%$ quantile $q_{50}$. Panel d) reports the network extracted by a not parametric methodology on the $50 \%$ quantile $q_{50}$. All the causality regression are computed without the market factor. 


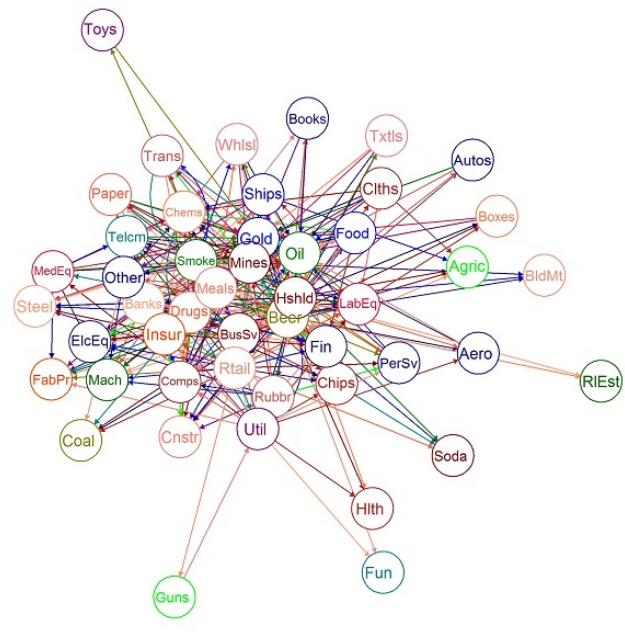

(a) Granger

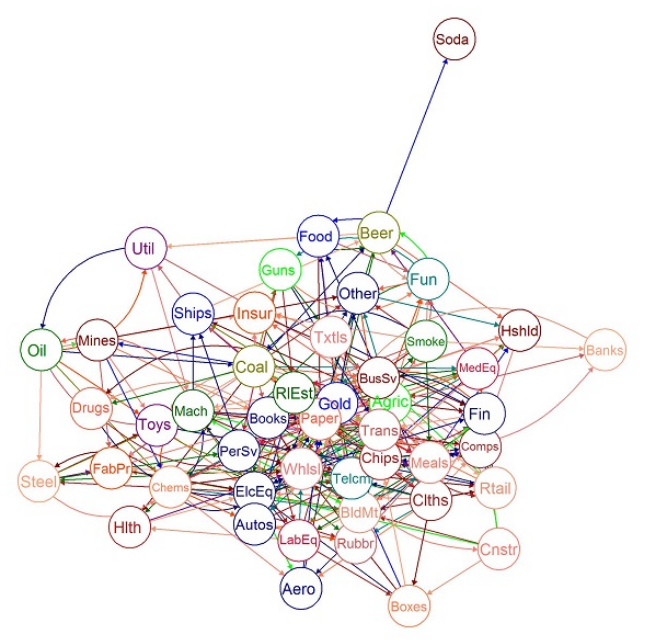

(c) $Q_{90}$ Quantile on Quantile

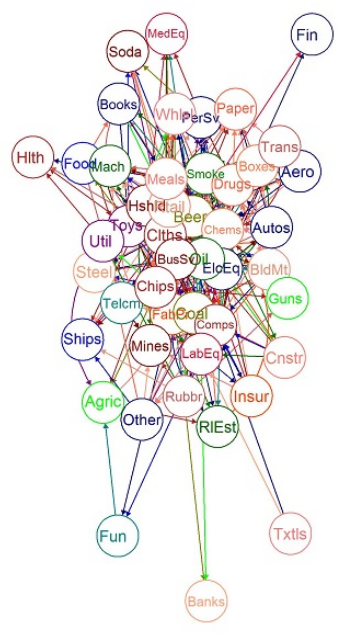

(b) $Q_{90}$ Base Quantile

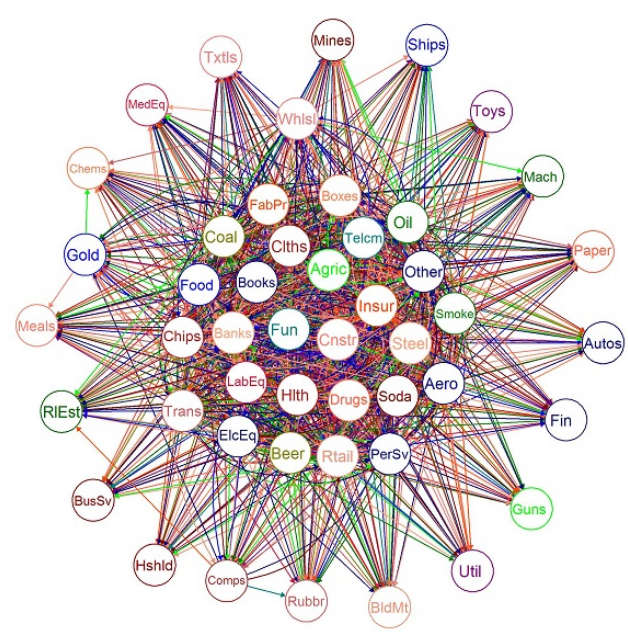

(d) $Q_{90}$ Not Parametric

Figure C.17: This figure visualizes 4 different networks for the period 2006-2008 relative to the 48 Fama and French industry portofolios. In this case the networks visualized are some of the primitive networks used for the resulting network computations. Panel a) reports the network extracted by the standard granger causality. Panel b) indicates the network extracted by a quantile baseline regression methodology on the $90 \%$ quantile $q_{90}$. Panel c) indicates the network extracted by a quantile on quantile methodology on the $90 \%$ quantile $q_{90}$. Panel d) reports the network extracted by a not parametric methodology on the $90 \%$ quantile $q_{90}$. All the causality regression are computed without the market factor. 


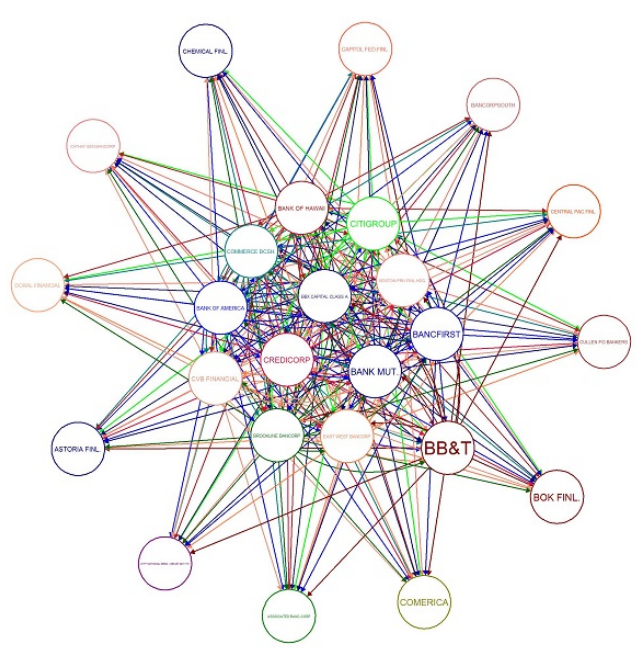

(a) $Q_{10}$ Not Parametric

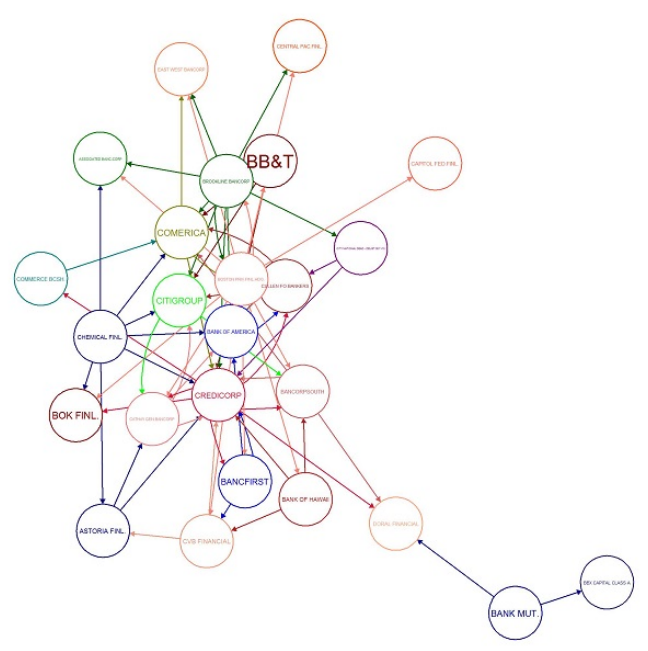

(c) $Q_{90}$ Base Quantile

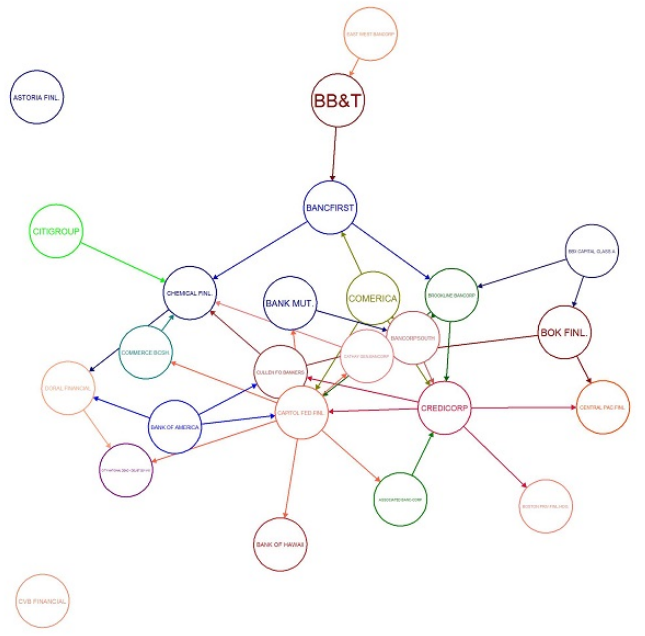

(b) $Q_{50}$ Quantile on Quantile

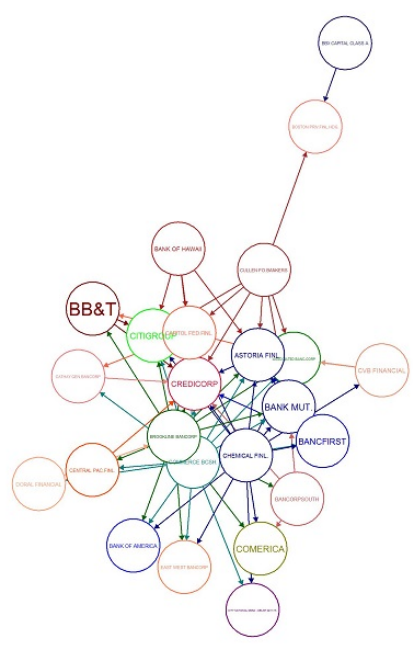

(d) Granger

Figure C.18: This figure visualizes 4 different networks for the period 2006-2008 relative to the first 25 banks ordered for market capitalization. In this case the networks visualized are some of the primitive networks used for the resulting network computations. Panel a) reports the network extracted by a Not parametric methodologie on the $10 \%$ quantile $q_{10}$. Panel b) indicates the network extracted by a quantile on quantile methodology on the median $q_{50}$. Panel c) displays the network extracted by a Not parametric methodologie on the $10 \%$ quantile $q_{10}$. Panel b) indicates the network extracted by a quantile on quantile methodology on the $90 \%$ quantile $q_{90}$. Panel d) reports the network extracted by the standard granger causality. All the causality regression are computed without the market factor. 


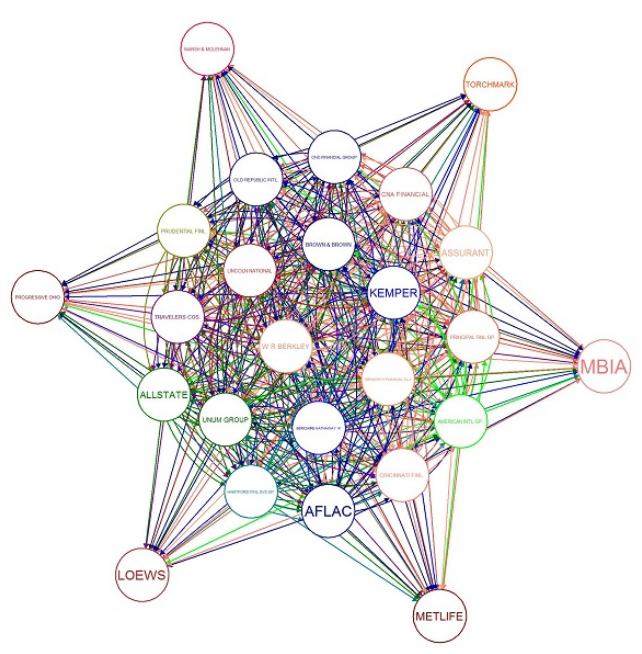

(a) $Q_{10}$ Not Parametric

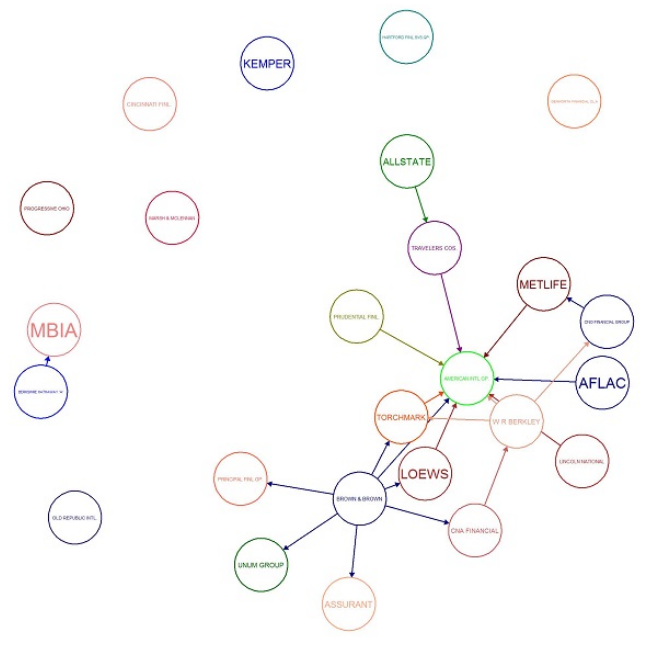

(c) $Q_{90}$ Base Quantile

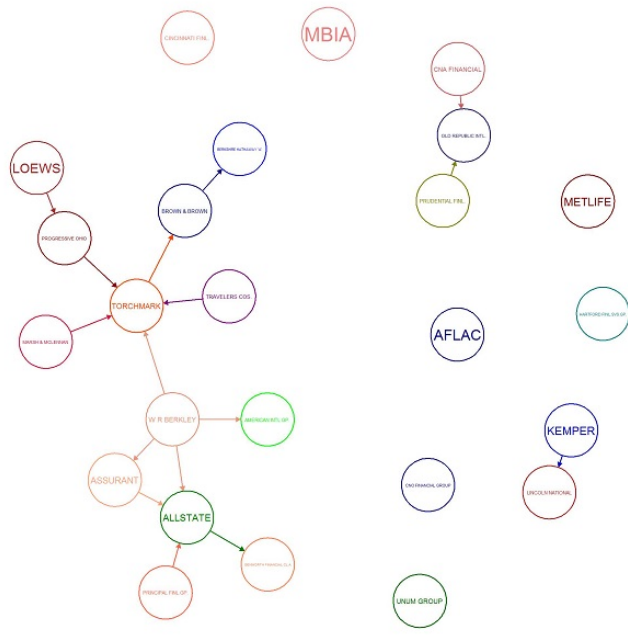

(b) $Q_{50}$ Quantile on Quantile

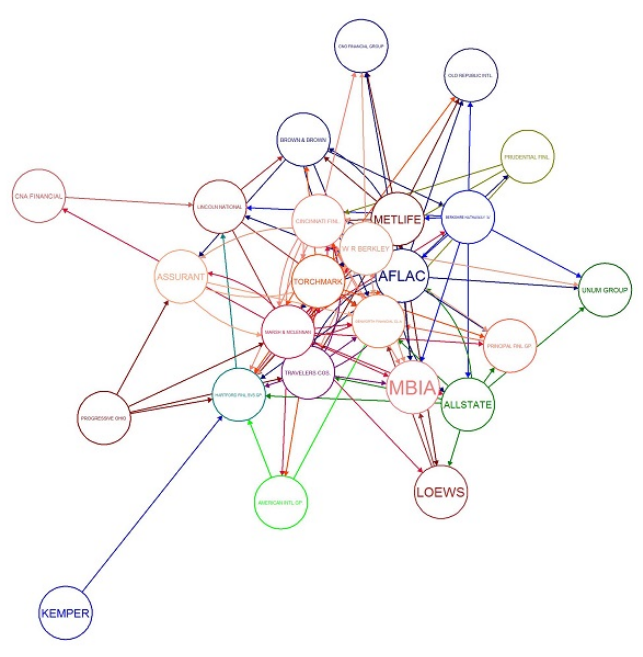

(d) Granger

Figure C.19: This figure visualizes 4 different networks for the period 2006-2008 relative to the first 25 Insurers ordered for market capitalization. In this case the networks visualized are some of the primitive networks used for the resulting network computations. Panel a) reports the network extracted by a Not parametric methodologie on the $10 \%$ quantile $q_{10}$. Panel b) indicates the network extracted by a quantile on quantile methodology on the median $q_{50}$. Panel c) displays the network extracted by a Not parametric methodologie on the $10 \%$ quantile $q_{10}$. Panel b) indicates the network extracted by a quantile on quantile methodology on the $90 \%$ quantile $q_{90}$. Panel d) reports the network extracted by the standard granger causality. All the causality regression are computed without the market factor. 


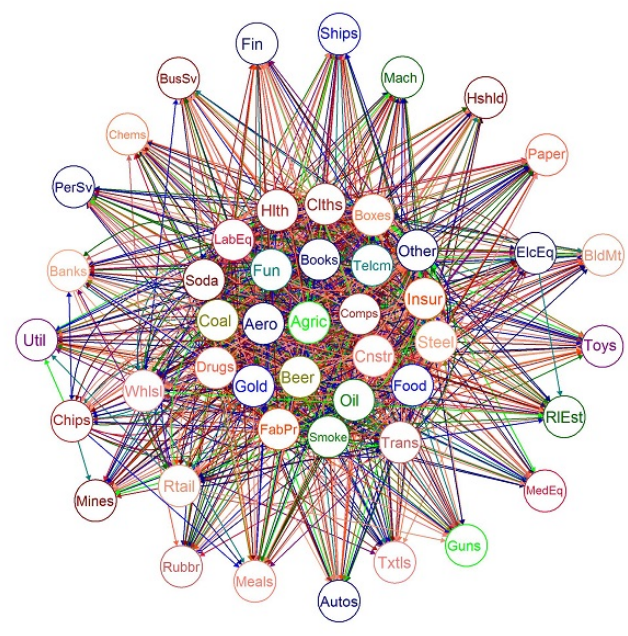

(a) $Q_{10}$ Not Parametric

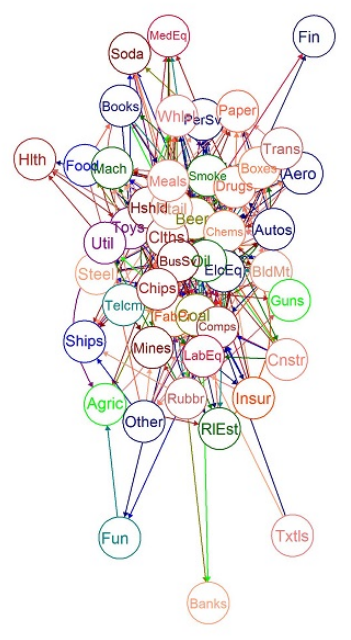

(c) $Q_{90}$ Base Quantile

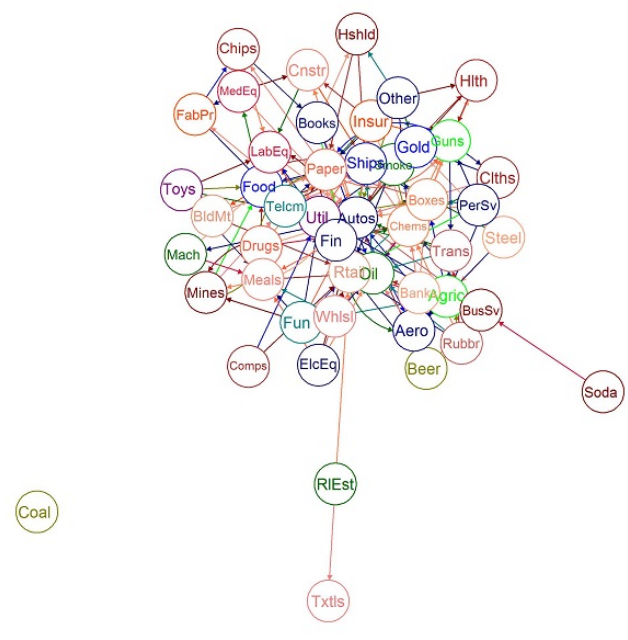

(b) $Q_{50}$ Quantile on Quantile

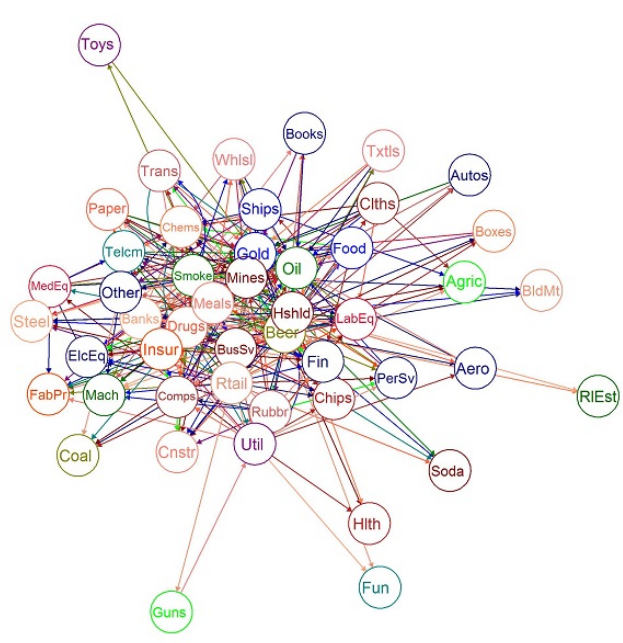

(d) Granger

Figure C.20: This figure visualizes 4 different networks for the period 2006-2008 relative to the first 48 Fama and French industry portfolios. In this case the networks visualized are some of the primitive networks used for the resulting network computations. Panel a) reports the network extracted by a Not parametric methodology on the $10 \%$ quantile $q_{10}$. Panel b) indicates the network extracted by a quantile on quantile methodology on the median $q_{50}$. Panel c) displays the network extracted by a Not parametric methodology on the $10 \%$ quantile $q_{10}$. Panel b) indicates the network extracted by a quantile on quantile methodology on the $90 \%$ quantile $q_{90}$. Panel d) reports the network extracted by the standard granger causality. All the causality regression are computed without the market factor. 


\section{Networks summary measures}

The network Density monitors the number of edges of the network relative to the maximum number of edges that the network might present. The Density equals $D=\frac{E}{V(V-1)}$ where $E$ is the total number of edges observed in a given network and $V$ is the number of nodes of the network. For further details we refer to Wasserman and Faust (1994).

The second measure we consider is Assortativity, denote by $r$, also called homophily, which captures the nodes tendency to connect with nodes having similar properties. In this work, we use a special type of assortativity, called assortativity by degree, see Newman $(2010)$ and Newman (2002). This measure monitors the node willingness to create links with nodes having similar degree. In this case, the degree $k_{i}, \quad i=1,2, \ldots V$, has a double role, the first (the standard one) represents the number of links ending at node $i$, the second one is the value assigned to that node $i$ for computing the assortativity. In the latter case, the degree might be a measure of the number of edges connecting the node to other nodes of the network, but could also be any continuous variable, for instance associated with the relevance of the edges. In order to distinguish between these two roles, we denote the degree by $k_{i}$ in the first case, and $x_{i}$ in the second case. Note that, the two values might be identical. We define the mean value $\mu$ of $x_{i}$ as:

$$
\mu=\frac{\sum_{i}^{K} \sum_{j}^{K} W_{i j} x_{i}}{2 E}=\frac{\sum_{i} k_{i} x_{i}}{2 E}
$$

where $2 E$ are the ends for all edges across the network, and $W_{i j}$ is the element of the unnormalized and un-weighted adjacency matrix $W$. The covariance between $x_{i}$ and $x_{j}$ is a way to measure the co-variation between node $i$ and node $j$ with respect to the variable $x$, which is, in the simplest case, the degree. Across the edges, this covariance can be formally defined as:

$$
\begin{aligned}
\operatorname{cov}\left(x_{i}, x_{j}\right) & =\frac{\sum_{i j} W_{i j}\left(x_{i}-\mu\right)\left(x_{j}-\mu\right)}{\sum_{i j} W_{i j}}=\frac{1}{2 E} \sum_{i j} W_{i j}\left(x_{i}-\mu\right)\left(x_{j}-\mu\right) \\
& =\frac{1}{2 E} \sum_{i j} W_{i j}\left(x_{i} x_{j}-\mu x_{i}-\mu x_{j}+\mu^{2}\right) \\
& =\frac{1}{2 E} \sum_{i j} W_{i j} x_{i} x_{j}-\frac{\sum_{i j} W_{i j} x_{i} \mu}{2 E}-\frac{\sum_{i j} W_{i j} x_{j} \mu}{2 E}-\frac{\sum_{i j} W_{i j} \mu^{2}}{2 E} \\
& =\frac{1}{2 E} \sum_{i j} W_{i j} x_{i} x_{j}-\mu^{2} \\
& =\frac{1}{2 E} \sum_{i j} W_{i j} x_{i} x_{j}-\frac{\sum_{i j} k_{i} k_{j} x_{i} x_{j}}{(2 E)^{2}}=\frac{1}{2 E} \sum_{i j}\left(W_{i j}-\frac{k_{i} k_{j}}{2 E}\right) x_{i} x_{j}
\end{aligned}
$$

In order to bound the assortativity coefficient in $-1 \leq r \leq 1$, the covariance is normalized by the maximum covariance value that can be reached by the network. The latter corresponds to the case where all the nodes share edges with nodes having the same degree (or $x_{i}=x_{j}$ ).

$$
\operatorname{cov}\left(x_{i}, x_{j}\right)_{\max }=\operatorname{cov}\left(x_{i}, x_{i}\right)=\frac{1}{2 E} \sum_{i j}\left(W_{i j}-\frac{k_{i} k_{j}}{2 E}\right) x_{i}^{2}
$$


Finally, as out interest lies in the evaluation of the assortativity by degree, we substitute $x_{i}$ and $x_{j}$ with $k_{i}$ and $k_{j}$, and obtain:

$$
r=\frac{\sum_{i j}\left(W_{i j}-k_{i} k_{j} / 2 E\right) k_{i} k_{j}}{\sum_{i j}\left(W_{i j}-k_{i} k_{j} / 2 E\right) k_{i}^{2}}
$$

If the $W$ matrix is not symmetric, then the network associated with this matrix is directed, and equation 30 must be modified ${ }^{7}$ In that case, $2 E$ becomes $\sum_{i j} W_{i j}=M$ and we distinguish the degree in indegree $k_{i}^{i n}$ and outdegree $k_{i}^{\text {out }}$, respectively, for the $i-t h$ node.

Formula 31 must be slightly modified. We are not using directly $k^{\text {in }}$ and $k^{\text {out }}$ but the excess degree, labeled with the symbol $e$. For an un-directed network, the excess degree or remaining degree is the number of edges leaving a given vertex minus one. As an example, if the $l-t h$ edge links the vertex $i$ with the vertex $j$, and the two vertexes have degree $k_{i}$ and $k_{j}$, respectively, then the excess degree for the $i_{t h}$ and $j_{t h}$ node is $e_{i}=k_{i}-1$ and $e_{j}=k_{j}-1$, respectively. If we have a directed network, each node has an excess outdegree $e^{\text {out }}$ and an excess indegree $e^{i n}$. Thus, if the $l-t h$ edge starts from vertex $i$ and goes to vertex $j$, then the excess outdegree for the $i-t h$ node is $e_{i}^{\text {out }}=k_{i}^{\text {out }}-1$ and excess indegree for the $j-$ th node is $e_{j}^{i n}=k_{j}^{i n}-1$.

In the case of a directed network, to compute the assortativity by degree we use:

$$
r=\frac{\sum_{i j} e_{i}^{i n} e_{j}^{\text {out }}-\frac{1}{M} \sum_{i} e_{i}^{\text {in }} \sum_{j} e_{j}^{\text {out }}}{\sqrt{\left(\sum_{i}\left(e_{i}^{i n}\right)^{2}-\frac{1}{M}\left(\sum_{i} e_{i}^{\text {in }}\right)^{2}\right)\left(\sum_{j}\left(e_{j}^{\text {out }}\right)^{2}-\frac{1}{M}\left(\sum_{j} e_{j}^{\text {out }}\right)^{2}\right)}}
$$

Since the assortativity measure is a ratio, indeterminate forms are also possible. The probability having indeterminate forms $\frac{0}{0}$ increases with a limited number of nodes and high number of edges. In this circumstances the actual number of links among the nodes is almost equal to that we would expect if the links were random, the effect is therefore the reduction of the numerator to zero. In addition, the almost completeness of the network makes null denominator because all the nodes have the same degree and consequently they behave as in a perfect assortativity mixing pattern (nodes with same degree are connected with node having exactly the same degree) where there is only one category given by the degree. Therefore, the indeterminateness arises for the impossibility to capture the network homophily tendency, because of the coexistence of two scenarios: a fully assortative pattern from one side and completely random from the other.

The last two measures we consider are closely related. The first, is the Eigenvector Centrality introduced by Bonacich (1987) This measure captures the nodes relevance (or centrality) as a function of the relevance of neighbor nodes. In other words, we make the prestige of the node proportional to the prestige of its neighbors. Formally, if we define with $x_{i}$ the prestige of the $i_{t h}$ node and $W$ the adjacency matrix, we assume that

$$
x_{i}=\sum_{j} W_{i j} x_{j}
$$

We can rewrite the previous expression 32 by using a matrix notation. We denote by $\mathbf{x}$

\footnotetext{
${ }^{7}$ For details see Newman $(2002)$ and Newman $\sqrt{2003) .}$
} 
the $V$-dimensional vector collecting the centrality score. The vector must satisfy the following equality:

$$
\mathbf{x}=W \mathbf{x}
$$

The estimation of the eigenvector centrality requires the use of an iterative procedure. In particular, starting from an initial guess for the centrality scores, $\mathbf{x}_{0}$, which we set equal to a vector of ones, the centrality scores are updated following

$$
\mathbf{x}_{t}=W \mathbf{x}_{t-1}=W^{t} \mathbf{x}_{0}
$$

where $t$ denotes th $t-t h$ iteration. The vector $\mathbf{x}_{0}$ can also be seen as a decomposition of linear independent vectors, where $c_{i}$ is the scalar associated with the $i-t h$ component.

$$
\mathbf{x}_{0}=\sum_{i}^{N} c_{i} \mathbf{v}_{i}
$$

If we plug equation 35 into equation 34 , by using the equivalence that $W \mathbf{x}=\lambda \mathbf{x}$ we obtain:

$$
\begin{aligned}
\mathbf{x}_{t} & =W^{t} \mathbf{x}_{0}=W^{t} \sum_{i}^{N} c_{i} \mathbf{v}_{i}=\sum_{i}^{N} c_{i} W^{t} \mathbf{v}_{i}=\sum_{i}^{N} c_{i} W^{t-1} \lambda_{i} \mathbf{v}_{i}=\sum_{i}^{N} c_{i} \lambda_{i}^{t} \mathbf{v}_{i} \\
& =\left(\frac{\lambda_{1}}{\lambda_{1}}\right)^{t} \sum_{i}^{N} c_{i} \lambda_{i}^{t} \mathbf{v}_{i}=\lambda_{1}^{t} \sum_{i}^{N} c_{i}\left(\frac{\lambda_{i}}{\lambda_{1}}\right)^{t} \mathbf{v}_{i}
\end{aligned}
$$

where $\lambda_{i}$ is the $i-t h$ eigenvalue associated to the $i-t h$ eigenvector, and $\lambda_{1}$ is the maximum eigenvalue 8 What we learn from the expression 37 is the following:

since $\lambda_{1}$ is the maximum eigenvalue, as soon $t \rightarrow \infty$ the $\sum_{i}^{N} c_{i}\left(\frac{\lambda_{i}}{\lambda_{1}}\right)^{t} \mathbf{v}_{i}$ tends to $c_{1} \mathbf{v}_{1}$ and thus the eigenvector centrality is proportional to the first eigenvalue as in the equation 37 we have 9

$$
\mathbf{x}_{t}=\lambda_{1}^{t} c_{1} \mathbf{v}_{1}
$$

In other words, combining the equation 37 with the 32 , we can observe that the centrality score is a function of the first eigenvalue

$$
x_{i}=\frac{1}{\lambda_{1}} \sum_{j} W_{i j} x_{j} .
$$

The eigenvector centrality can be computed not only for adjacency matrix $W$ but also for the normalized adjacency matrix.

\footnotetext{
${ }^{8}$ The existence of the maximum eigenvalue is guaranteed by the Perron- Frobenius theorem.

${ }^{9}$ The eigenvector centrality does not converge when the maximum eigenvalue $\lambda_{1}$ tends to zero. The maximum eigenvalue coefficient decreases with the sparsity of the adjacency matrix (Van Mieghem, 2010)
} 


\section{E Figures of Combined Network}

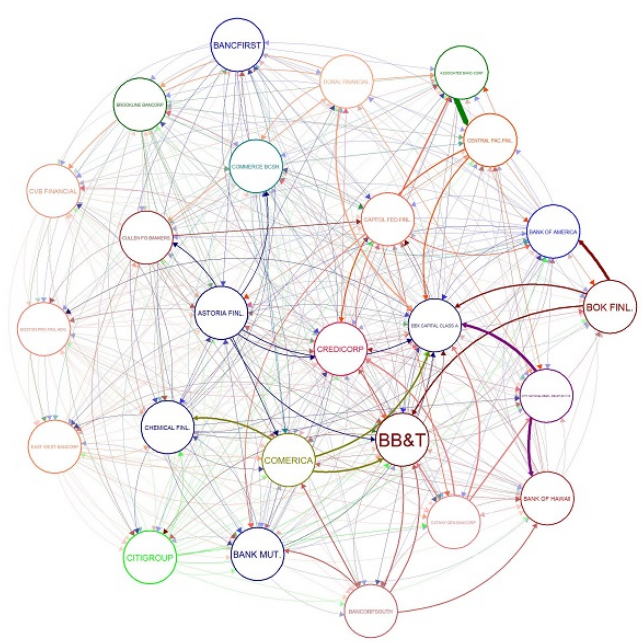

(a) $Q_{10}$

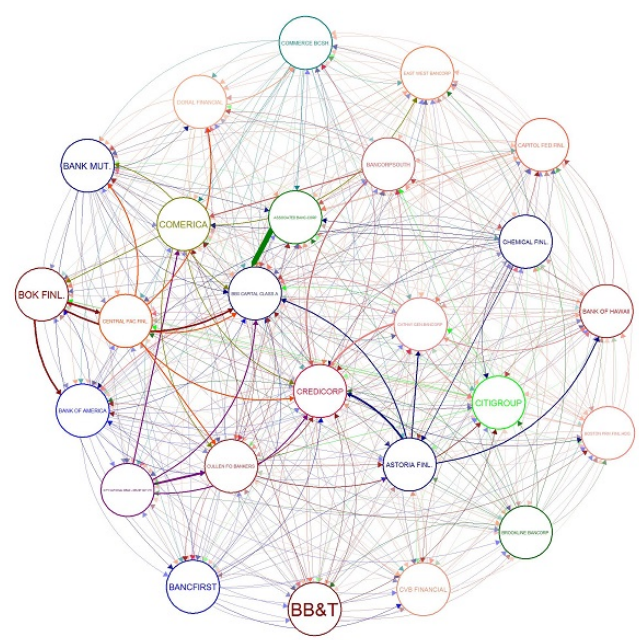

(c) $Q_{90}$

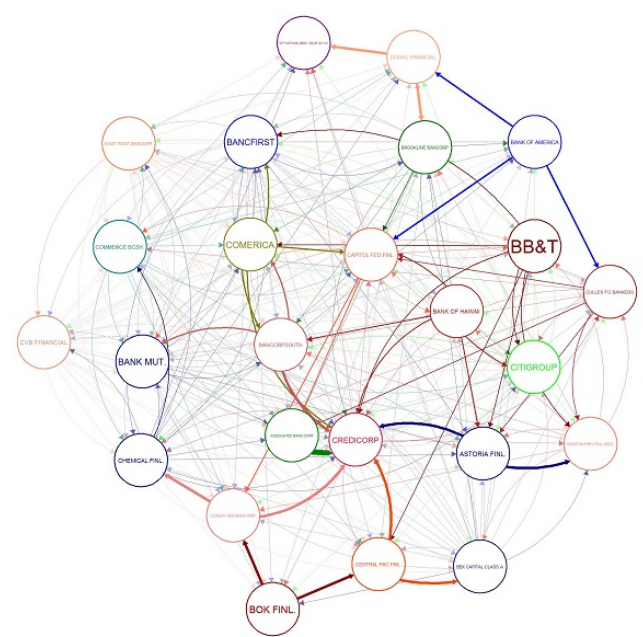

(b) $Q_{50}$

Figure E.21: This figure visualizes the 3 different resulting networks for the period 2006-2008 relative to the first 25 banks ordered for market capitalization. Panel a) reports the network extracted by combining causality network by using quantile regression (QB, Qo and QN) at the $10 \%$ quantile, and the standard granger causality method. Panel b) reports the network extracted by combining causality network by using quantile regression (QB, Qo and QN) at the $50 \%$ quantile, and the standard granger causality method. Panel c) reports the network extracted by combining causality network by using quantile regression (QB, Qo and QN) at the $90 \%$ quantile, and the standard granger causality method. All the causality regression are computed without the market factor. 


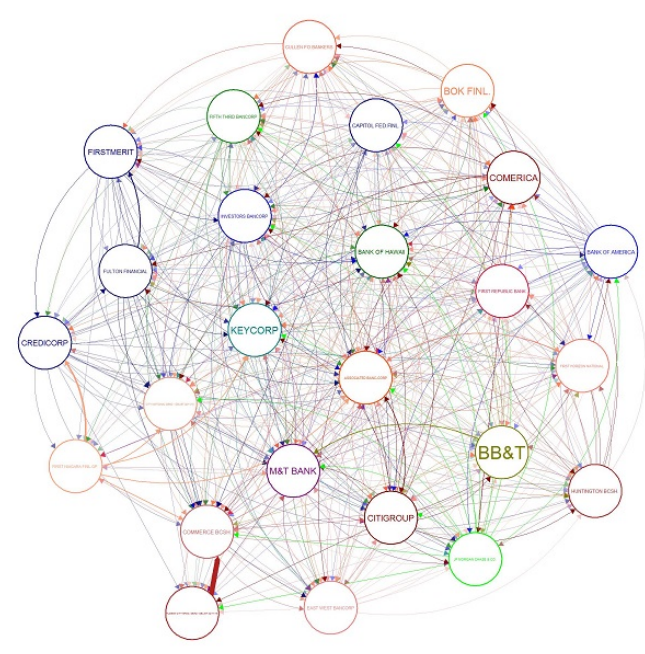

(a) $Q_{10}$

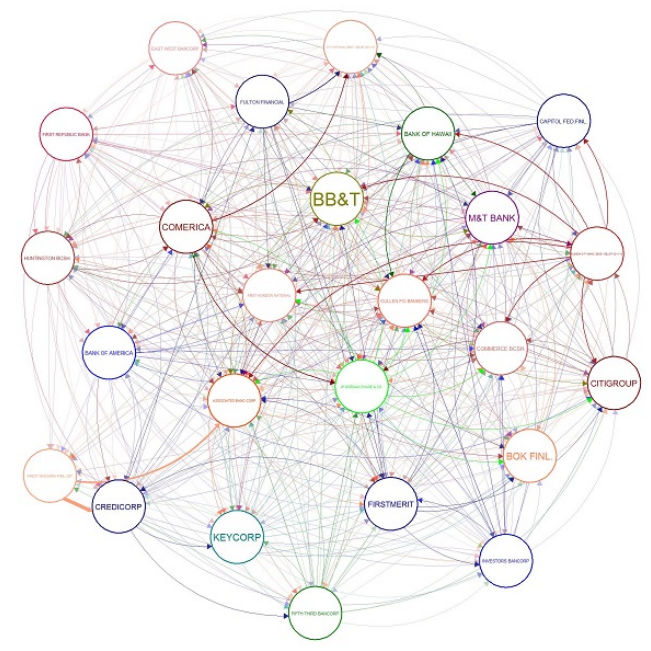

(c) $Q_{90}$

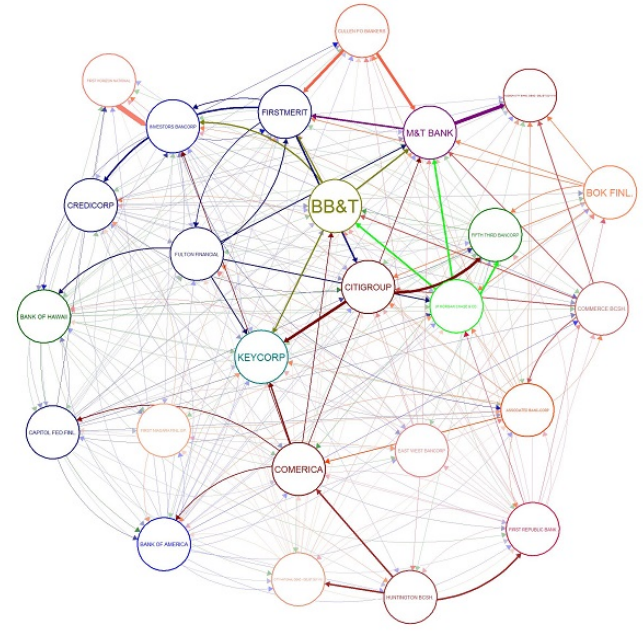

(b) $Q_{50}$

Figure E.22: This figure visualizes the 3 different resulting networks for the period 2011-2015 relative to the first 25 banks ordered for market capitalization. Panel a) reports the network extracted by combining causality network by using quantile regression (QB, Qo and QN) at the $10 \%$ quantile, and the standard granger causality method. Panel b) reports the network extracted by combining causality network by using quantile regression (QB, Qo and QN) at the $50 \%$ quantile, and the standard granger causality method. Panel c) reports the network extracted by combining causality network by using quantile regression (QB, Qo and $\mathrm{QN}$ ) at the $90 \%$ quantile, and the standard granger causality method. All the causality regression are computed without the market factor. 


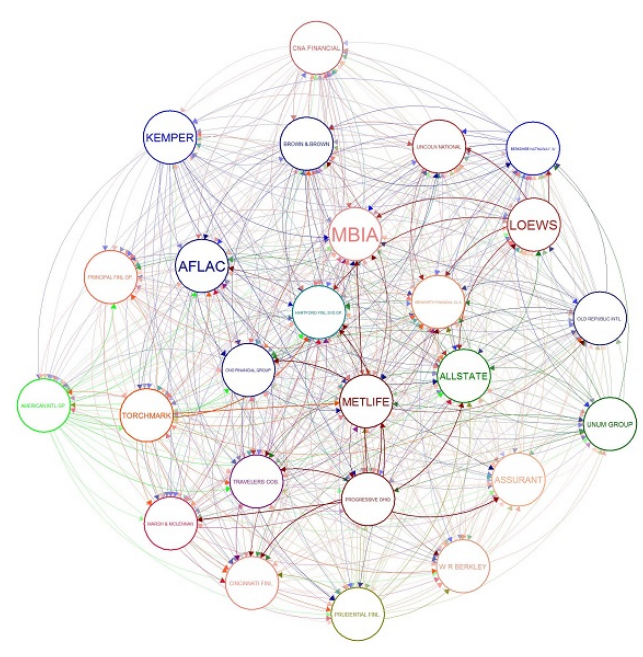

(a) $Q_{10}$

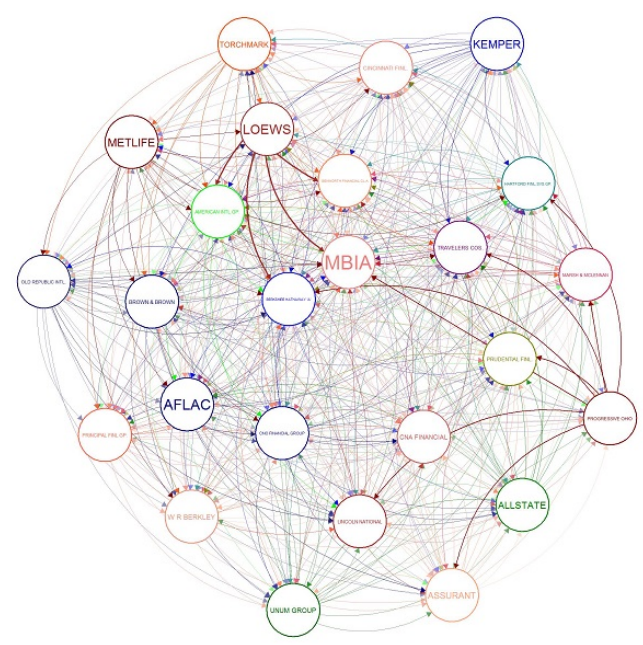

(c) $Q_{90}$

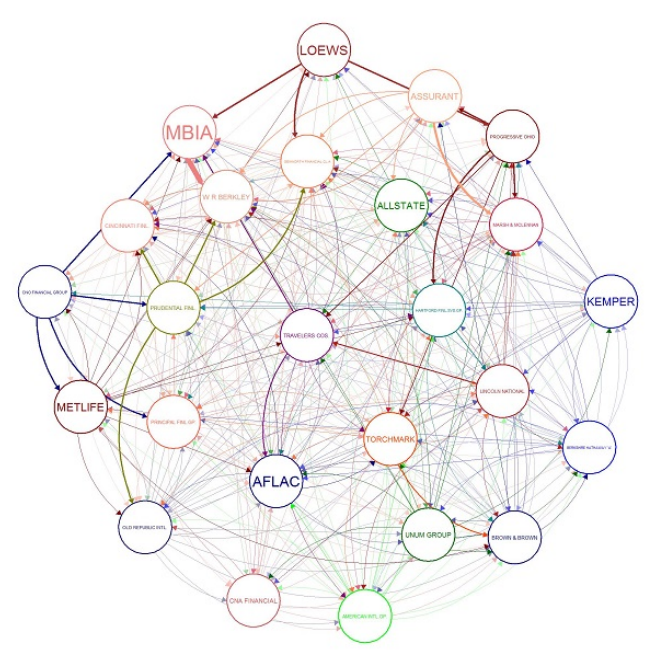

(b) $Q_{50}$

Figure E.23: This figure visualizes the 3 different resulting networks for the period 2006-2008 relative to the first 25 Insurance companies ordered for market capitalization. Panel a) reports the network extracted by combining causality network by using quantile regression (QB, Qo and $\mathrm{QN}$ ) at the $10 \%$ quantile, and the standard granger causality method. Panel b) reports the network extracted by combining causality network by using quantile regression (QB, Qo and QN) at the $50 \%$ quantile, and the standard granger causality method. Panel c) reports the network extracted by combining causality network by using quantile regression (QB, Qo and QN) at the $90 \%$ quantile, and the standard granger causality method. All the causality regression are computed without the market factor. 


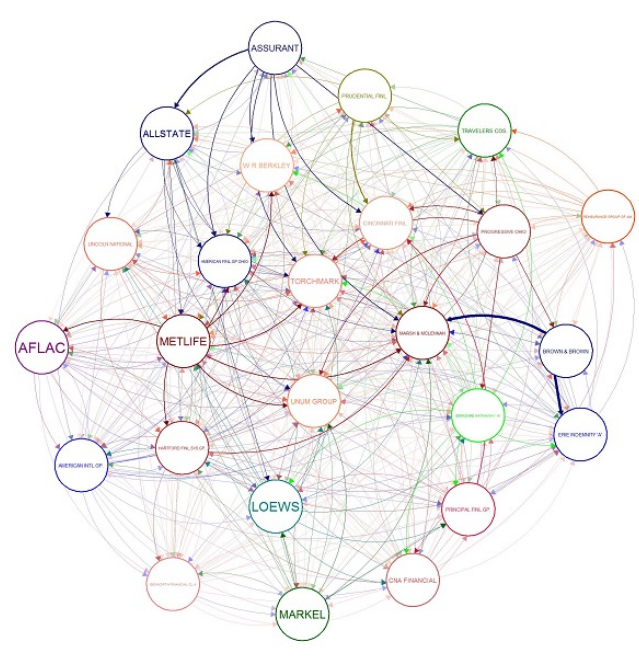

(a) $Q_{10}$

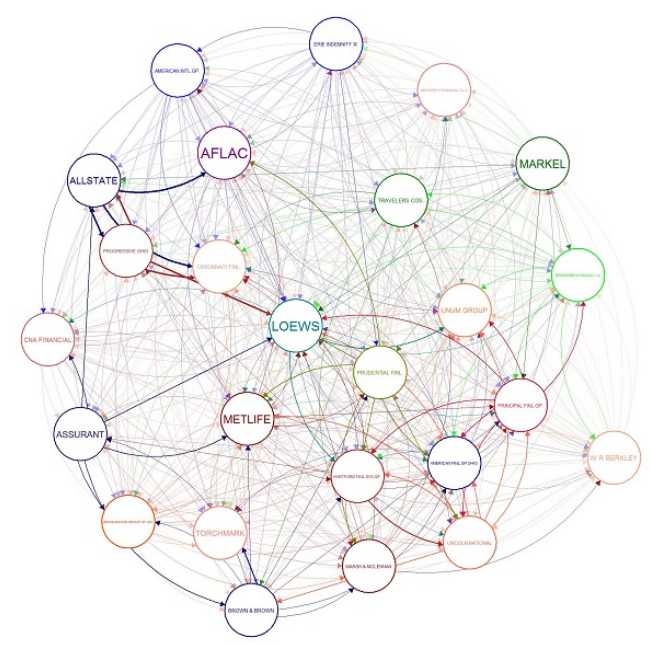

(c) $Q_{90}$

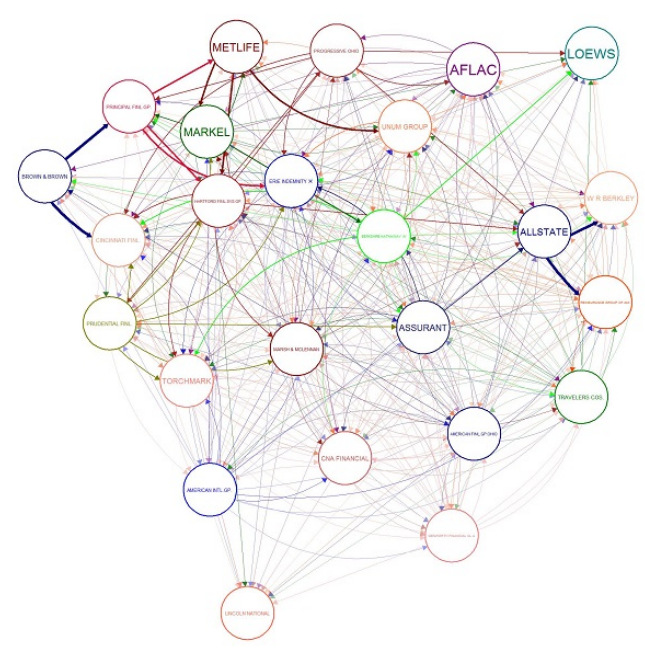

(b) $Q_{50}$

Figure E.24: This figure visualizes the 3 different resulting networks for the period 2011-2015 relative to the first 25 Insurance companies ordered for market capitalization. Panel a) reports the network extracted by combining causality network by using quantile regression (QB, Qo and $\mathrm{QN}$ ) at the $10 \%$ quantile, and the standard granger causality method. Panel b) reports the network extracted by combining causality network by using quantile regression (QB, Qo and $\mathrm{QN}$ ) at the $50 \%$ quantile, and the standard granger causality method. Panel c) reports the network extracted by combining causality network by using quantile regression (QB, Qo and $\mathrm{QN}$ ) at the $90 \%$ quantile, and the standard granger causality method. All the causality regression are computed without the market factor. 


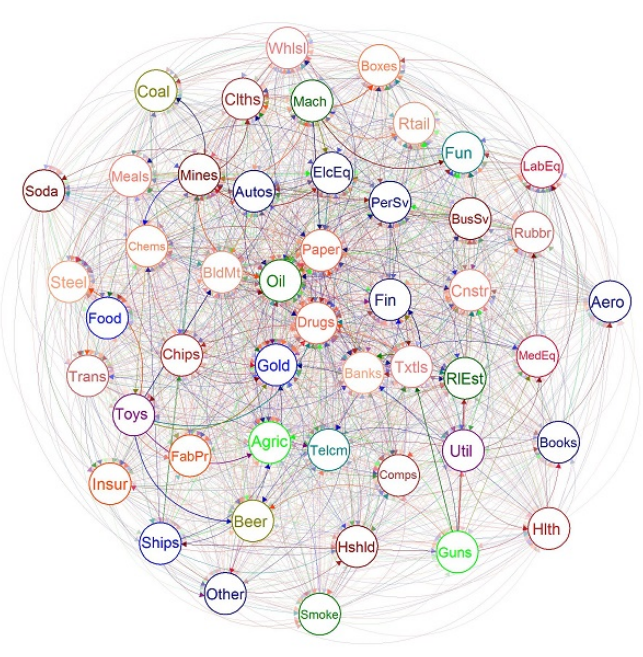

(a) $Q_{10}$

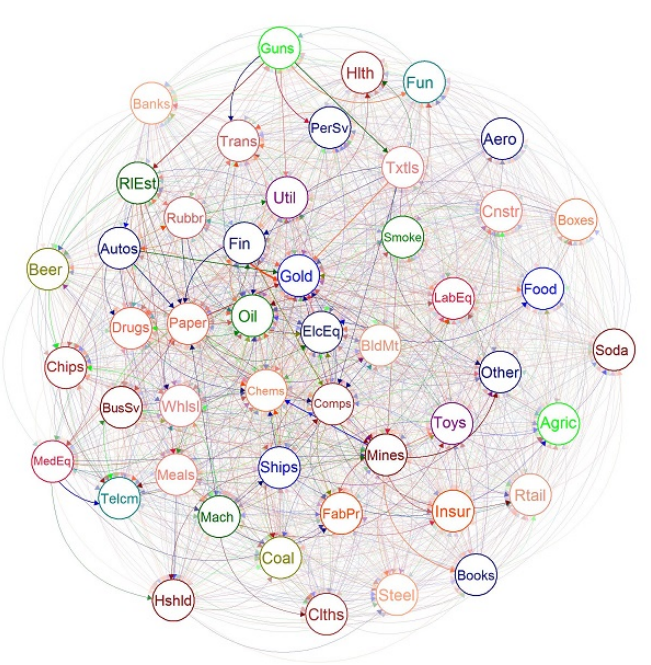

(c) $Q_{90}$

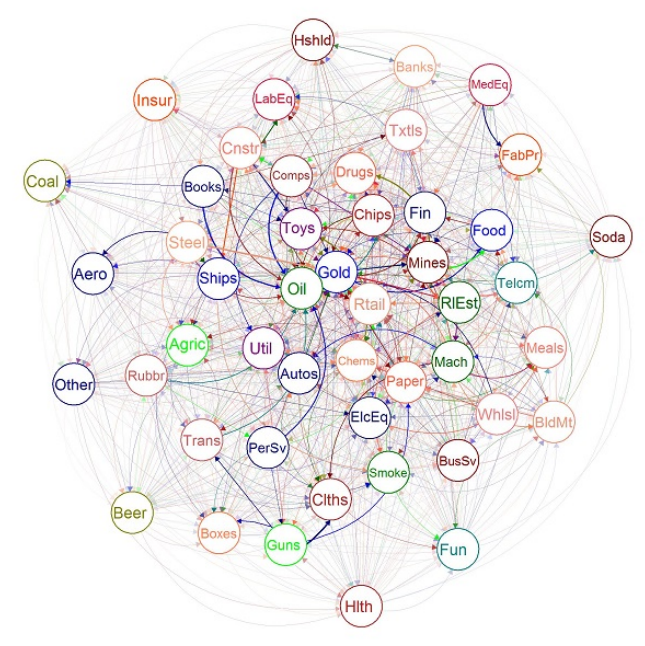

(b) $Q_{50}$

Figure E.25: This figure visualizes the 3 different resulting networks for the period 2006-2008 relative to 48 Industry portfolios. Panel a) reports the network extracted by combining causality network by using quantile regression (QB, Qo and QN) at the $10 \%$ quantile, and the standard granger causality method. Panel b) reports the network extracted by combining causality network by using quantile regression (QB, Qo and QN) at the $50 \%$ quantile, and the standard granger causality method. Panel c) reports the network extracted by combining causality network by using quantile regression (QB, Qo and $\mathrm{QN}$ ) at the $90 \%$ quantile, and the standard granger causality method. All the causality regression are computed without the market factor. 


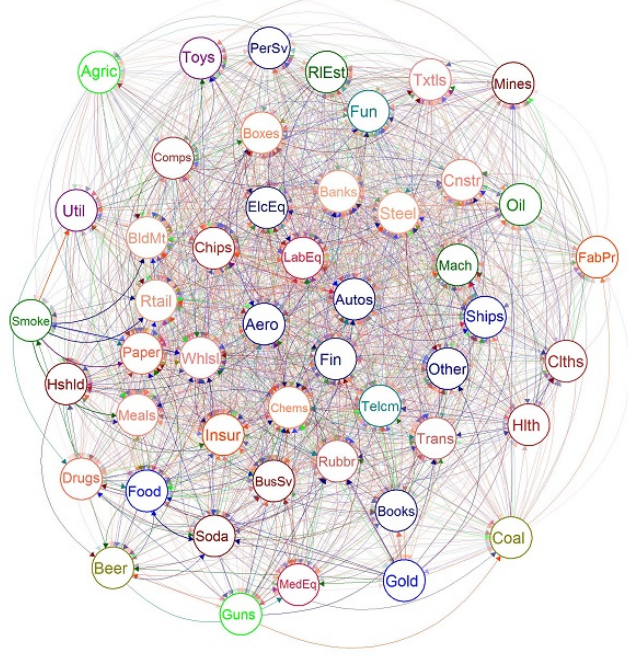

(a) $Q_{10}$

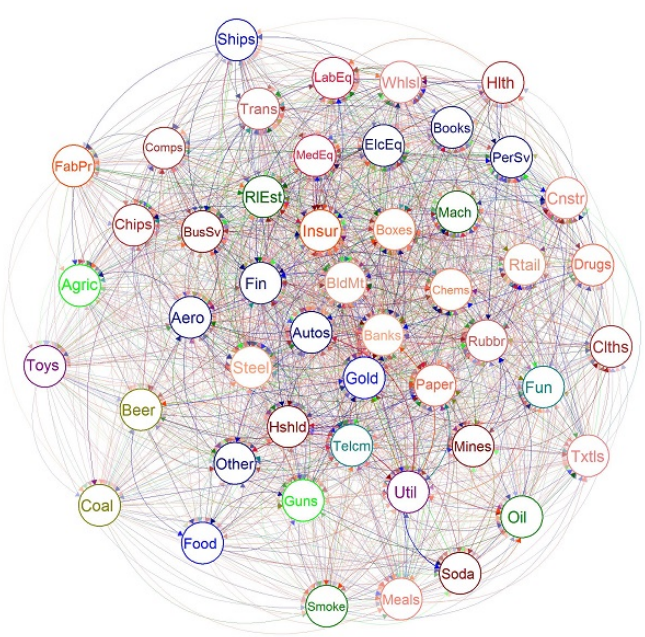

(c) $Q_{90}$

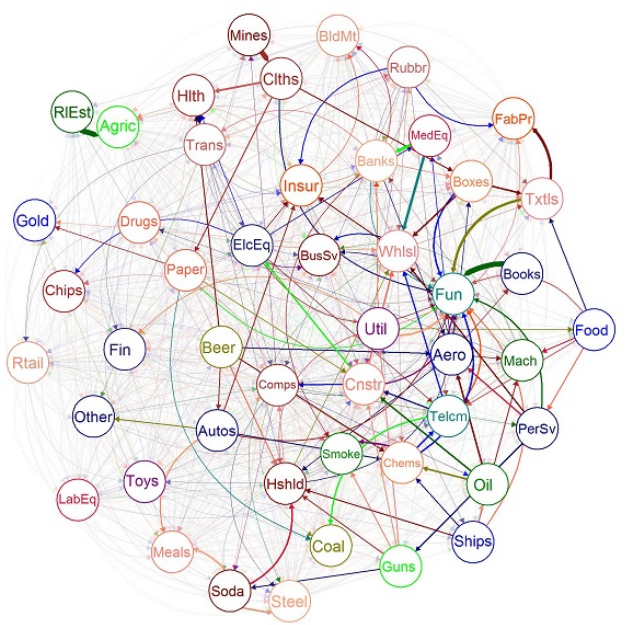

(b) $Q_{50}$

Figure E.26: This figure visualizes the 3 different resulting networks for the period 2011-2015 relative to 48 Industry portfolios. Panel a) reports the network extracted by combining causality network by using quantile regression (QB, Qo and QN) at the $10 \%$ quantile, and the standard granger causality method. Panel b) reports the network extracted by combining causality network by using quantile regression (QB, Qo and QN) at the $50 \%$ quantile, and the standard granger causality method. Panel c) reports the network extracted by combining causality network by using quantile regression (QB, Qo and $\mathrm{QN}$ ) at the $90 \%$ quantile, and the standard granger causality method. All the causality regression are computed without the market factor. 


\section{F Sensitivity analysis for weekly Returns}

Table F.15: The table reports the $\delta$ of model (5) that represent the weights for networks combination. The top panel focused on the banks dataset, the middle panel on the insurance companies dataset and the bottom panel on the industry portfolios dataset. The first column identifies the quantiles used to estimate the quantile-based network, and the second column indicates if a common factor was used $(\mathrm{Y})$ or not used $(\mathrm{N})$ in the estimation of the causality networks. Columns 3 to 5 refer to the crisis sample while columns 6 to 8 to the most recent sample. The second row identifies the three different networks which are optimally combined: baseline quantile causality - QB; quantile-on-quantile causality Qo; non-parametric quantile causality - QN. Parameters are, by construction, positive and sum up to one (within each row and within each period). A star identifies parameters significant at the $5 \%$ confidence level.

\begin{tabular}{|c|c|c|c|c|c|c|c|}
\hline \multirow[b]{2}{*}{ Quantile } & \multirow[b]{2}{*}{ Factor } & \multicolumn{3}{|c|}{ 2006-2008 } & \multicolumn{3}{|c|}{$2010-2015$} \\
\hline & & QB & Qo & QN & QB & Qo & QN \\
\hline \multicolumn{8}{|c|}{25 Banks } \\
\hline $10 \%$ & $\mathrm{~N}$ & 0.060 & $0.054^{*}$ & $0.885^{*}$ & 0.001 & 0.625 & 0.374 \\
\hline $50 \%$ & $\mathrm{~N}$ & 0.142 & $0.108^{*}$ & $0.750^{*}$ & 0.094 & 0.067 & $0.838^{*}$ \\
\hline $90 \%$ & $\mathrm{~N}$ & 0.028 & 0.026 & $0.946^{*}$ & 0.000 & $0.725^{*}$ & $0.275^{*}$ \\
\hline $10 \%$ & $\mathrm{Y}$ & 0.024 & 0.109 & $0.868^{*}$ & $0.000^{*}$ & $0.526^{*}$ & $0.474^{*}$ \\
\hline $50 \%$ & $\mathrm{Y}$ & 0.054 & $0.062^{*}$ & $0.885^{*}$ & 0.201 & $0.066^{*}$ & $0.733^{*}$ \\
\hline $90 \%$ & $\mathrm{Y}$ & 0.123 & 0.020 & $0.857^{*}$ & 0.000 & $0.877^{*}$ & $0.123^{*}$ \\
\hline \multicolumn{8}{|c|}{25 Insurance Companies } \\
\hline $10 \%$ & $\mathrm{~N}$ & 0.000 & 0.034 & $0.966^{*}$ & 0.058 & $0.831^{*}$ & $0.111^{*}$ \\
\hline $50 \%$ & $\mathrm{~N}$ & $0.842^{*}$ & 0.125 & 0.034 & 0.059 & 0.045 & $0.896^{*}$ \\
\hline $90 \%$ & $\mathrm{~N}$ & 0.000 & 0.028 & $0.972^{*}$ & 0.000 & $0.456^{*}$ & $0.544^{*}$ \\
\hline $10 \%$ & $\mathrm{Y}$ & 0.001 & 0.028 & $0.972^{*}$ & 0.000 & $0.488^{*}$ & $0.512^{*}$ \\
\hline $50 \%$ & $\mathrm{Y}$ & $0.678^{*}$ & 0.106 & $0.216^{*}$ & 0.053 & 0.041 & $0.907^{*}$ \\
\hline $90 \%$ & $\mathrm{Y}$ & 0.000 & 0.046 & 0.954 & 0.000 & 0.554 & 0.446 \\
\hline \multicolumn{8}{|c|}{48 Industry Portfolio } \\
\hline $10 \%$ & $\mathrm{~N}$ & 0.000 & $1.000^{*}$ & 0.000 & 0.032 & $0.968^{*}$ & 0.000 \\
\hline $50 \%$ & $\mathrm{~N}$ & 0.202 & 0.000 & 0.798 & 0.214 & 0.023 & 0.763 \\
\hline $90 \%$ & $\mathrm{~N}$ & 0.108 & $0.892^{*}$ & 0.000 & 0.016 & $0.984^{*}$ & 0.000 \\
\hline $10 \%$ & $\mathrm{Y}$ & 0.390 & 0.540 & 0.071 & 0.038 & $0.755^{*}$ & 0.207 \\
\hline $50 \%$ & $\mathrm{Y}$ & 0.237 & 0.613 & 0.150 & 0.089 & 0.024 & $0.888^{*}$ \\
\hline $90 \%$ & $\mathrm{Y}$ & 0.000 & $1.000^{*}$ & 0.000 & 0.111 & $0.857^{*}$ & 0.032 \\
\hline
\end{tabular}


Table F.16: The table reports residual correlation descriptive analyses for the Banks dataset. The first column identify the various models, while the second column indicates the number of networks used in the model. In the first column $Q(10 \%)$ identifies the use of a combination of causality networks from quantile regression (QB, Qo and QN) at the $10 \%$ quantile. Similarly, when the reference quantile is $50 \%$ or $90 \%$. With $G$ we denote the model using just the Granger casuality network, while the last line refers to the 4-factor CAPM. The table reports statistics for the residuals correlations: the minimum, maximum, the $10 \%$ quantile $q_{10}$, the median $q_{50}$, the $90 \%$ quantile and the number of elements of the correlation matrix lower than -0.1 .

\begin{tabular}{|c|c|c|c|c|c|c|c|c|}
\hline \multicolumn{9}{|c|}{$2006-2008$} \\
\hline Model & $\begin{array}{c}\mathrm{N} \\
\text { Networks }\end{array}$ & Factor & Min & $\operatorname{Max}$ & q10 & q50 & $\mathrm{q} 90$ & $\begin{array}{c}\% \text { elements } \\
\leq-0.1\end{array}$ \\
\hline $\mathrm{Q}(10 \%)$ & 4 & $\mathrm{~N}$ & -0.279 & 0.584 & -0.108 & 0.071 & 0.279 & $11.3 \%$ \\
\hline $\mathrm{Q}(50 \%)$ & 4 & $\mathrm{~N}$ & -0.344 & 0.597 & -0.106 & 0.060 & 0.316 & $11.0 \%$ \\
\hline $\mathrm{Q}(90 \%)$ & 4 & $\mathrm{~N}$ & -0.297 & 0.597 & -0.127 & 0.062 & 0.299 & $14.0 \%$ \\
\hline $\mathrm{Q}(10 \%)$ & 4 & $\mathrm{Y}$ & -0.291 & 0.567 & -0.135 & 0.046 & 0.253 & $16.0 \%$ \\
\hline $\mathrm{Q}(50 \%)$ & 4 & $\mathrm{Y}$ & -0.346 & 0.479 & -0.118 & 0.055 & 0.251 & $11.7 \%$ \\
\hline $\mathrm{Q}(90 \%)$ & 4 & $\mathrm{Y}$ & -0.301 & 0.533 & -0.137 & 0.038 & 0.235 & $16.3 \%$ \\
\hline $\mathrm{G}$ & 1 & $\mathrm{~N}$ & -0.381 & 0.670 & -0.128 & 0.111 & 0.372 & $13.3 \%$ \\
\hline $\mathrm{G}$ & 1 & $\mathrm{Y}$ & -0.394 & 0.641 & -0.128 & 0.089 & 0.378 & $11.7 \%$ \\
\hline 4-F-CAPM & 0 & N.A & -0.358 & 0.678 & -0.108 & 0.257 & 0.502 & $10.7 \%$ \\
\hline \multicolumn{9}{|c|}{ 2011-2015 } \\
\hline Model & $\begin{array}{c}\mathrm{N} \\
\text { Networks }\end{array}$ & Factor & Min & $\operatorname{Max}$ & q10 & q50 & q90 & $\begin{array}{c}\% \text { elements } \\
\leq-0.1\end{array}$ \\
\hline $\mathrm{Q}(10 \%)$ & 4 & $\mathrm{~N}$ & -0.265 & 0.424 & -0.121 & -0.015 & 0.137 & $14.7 \%$ \\
\hline $\mathrm{Q}(50 \%)$ & 4 & $\mathrm{~N}$ & -0.390 & 0.643 & -0.107 & 0.053 & 0.282 & $13.3 \%$ \\
\hline $\mathrm{Q}(90 \%)$ & 4 & $\mathrm{~N}$ & -0.248 & 0.432 & -0.125 & 0.010 & 0.136 & $15.3 \%$ \\
\hline $\mathrm{Q}(10 \%)$ & 4 & $\mathrm{Y}$ & -0.281 & 0.505 & -0.131 & -0.011 & 0.145 & $18.7 \%$ \\
\hline $\mathrm{Q}(50 \%)$ & 4 & $\mathrm{Y}$ & -0.316 & 0.609 & -0.127 & 0.054 & 0.245 & $15.0 \%$ \\
\hline $\mathrm{Q}(90 \%)$ & 4 & $\mathrm{Y}$ & -0.304 & 0.508 & -0.139 & 0.001 & 0.132 & $17.7 \%$ \\
\hline G & 1 & $\mathrm{~N}$ & -0.211 & 0.598 & -0.027 & 0.151 & 0.362 & $3.0 \%$ \\
\hline $\mathrm{G}$ & 1 & $\mathrm{Y}$ & -0.211 & 0.598 & -0.027 & 0.146 & 0.361 & $3.3 \%$ \\
\hline 4-F-CAPM & 0 & NA & -0.122 & 0.669 & 0.054 & 0.266 & 0.487 & $0.3 \%$ \\
\hline
\end{tabular}


Table F.17: The table reports residual correlation descriptive analyses for the Insurance Companies dataset. The first column identify the various models, while the second column indicates the number of networks used in the model. In the first column $Q(10 \%)$ identifies the use of a combination of causality networks from quantile regression (QB, Qo and QN) at the $10 \%$ quantile. Similarly, when the reference quantile is $50 \%$ or $90 \%$. With $G$ we denote the model using just the Granger casuality network, while the last line refers to the 4-factor CAPM. The table reports statistics for the residuals correlations: the minimum, maximum, the $10 \%$ quantile $q_{10}$, the median $q_{50}$, the $90 \%$ quantile and the number of elements of the correlation matrix lower than -0.1 .

\begin{tabular}{|c|c|c|c|c|c|c|c|c|}
\hline \multicolumn{9}{|c|}{ 2006-2008 } \\
\hline Model & $\begin{array}{c}\mathrm{N} \\
\text { Networks }\end{array}$ & Factor & Min & $\operatorname{Max}$ & q10 & q50 & q90 & $\begin{array}{c}\% \text { elements } \\
\leq-0.1\end{array}$ \\
\hline $\mathrm{Q}(10 \%)$ & 4 & $\mathrm{~N}$ & -0.440 & 0.682 & -0.222 & 0.002 & 0.248 & $27.0 \%$ \\
\hline $\mathrm{Q}(50 \%)$ & 4 & $\mathrm{~N}$ & -0.423 & 0.694 & -0.161 & 0.044 & 0.260 & $16.7 \%$ \\
\hline $\mathrm{Q}(90 \%)$ & 4 & $\mathrm{~N}$ & -0.448 & 0.676 & -0.207 & 0.009 & 0.255 & $25.7 \%$ \\
\hline $\mathrm{Q}(10 \%)$ & 4 & $\mathrm{Y}$ & -0.455 & 0.686 & -0.212 & 0.002 & 0.257 & $26.7 \%$ \\
\hline $\mathrm{Q}(50 \%)$ & 4 & $\mathrm{Y}$ & -0.369 & 0.515 & -0.181 & 0.018 & 0.225 & $20.7 \%$ \\
\hline $\mathrm{Q}(90 \%)$ & 4 & $\mathrm{Y}$ & -0.464 & 0.671 & -0.214 & 0.001 & 0.253 & $27.0 \%$ \\
\hline G & 1 & $\mathrm{~N}$ & -0.466 & 0.831 & -0.166 & 0.044 & 0.316 & $17.7 \%$ \\
\hline G & 1 & Y & -0.460 & 0.714 & -0.166 & 0.048 & 0.323 & $15.0 \%$ \\
\hline 4-F-CAPM & 0 & N.A & -0.370 & 0.847 & -0.149 & 0.089 & 0.422 & $14.0 \%$ \\
\hline \multicolumn{9}{|c|}{ 2011-2015 } \\
\hline Model & $\begin{array}{c}\mathrm{N} \\
\text { Networks }\end{array}$ & Factor & Min & $\operatorname{Max}$ & q10 & q50 & q90 & $\begin{array}{c}\% \text { elements } \\
\leq-0.1\end{array}$ \\
\hline $\mathrm{Q}(10 \%)$ & 4 & $\mathrm{~N}$ & -0.290 & 0.504 & -0.121 & 0.009 & 0.157 & $14.7 \%$ \\
\hline $\mathrm{Q}(50 \%)$ & 4 & $\mathrm{~N}$ & -0.258 & 0.547 & -0.149 & 0.019 & 0.202 & $16.7 \%$ \\
\hline $\mathrm{Q}(90 \%)$ & 4 & $\mathrm{~N}$ & -0.289 & 0.345 & -0.140 & -0.004 & 0.168 & $18.3 \%$ \\
\hline $\mathrm{Q}(10 \%)$ & 4 & $\mathrm{Y}$ & -0.333 & 0.503 & -0.153 & -0.012 & 0.189 & $20.7 \%$ \\
\hline $\mathrm{Q}(50 \%)$ & 4 & $\mathrm{Y}$ & -0.268 & 0.590 & -0.157 & 0.019 & 0.191 & $18.3 \%$ \\
\hline $\mathrm{Q}(90 \%)$ & 4 & $\mathrm{Y}$ & -0.299 & 0.398 & -0.152 & -0.001 & 0.199 & $21.3 \%$ \\
\hline G & 1 & $\mathrm{~N}$ & -0.136 & 0.658 & -0.005 & 0.121 & 0.317 & $1.7 \%$ \\
\hline $\mathrm{G}$ & 1 & $\mathrm{Y}$ & -0.136 & 0.658 & -0.014 & 0.116 & 0.315 & $1.7 \%$ \\
\hline 4-F-CAPM & 0 & N.A & -0.103 & 0.658 & 0.017 & 0.138 & 0.332 & $0.3 \%$ \\
\hline
\end{tabular}


Table F.18: The table reports residual correlation descriptive analyses for the Industry portfolios dataset. The first column identify the various models, while the second column indicates the number of networks used in the model. In the first column $Q(10 \%)$ identifies the use of a combination of causality networks from quantile regression (QB, Qo and QN) at the $10 \%$ quantile. Similarly, when the reference quantile is $50 \%$ or $90 \%$. With $G$ we denote the model using just the Granger casuality network, while the last line refers to the 4-factor CAPM. The table reports statistics for the residuals correlations: the minimum, maximum, the $10 \%$ quantile $q_{10}$, the median $q_{50}$, the $90 \%$ quantile and the number of elements of the correlation matrix lower than -0.1 .

\begin{tabular}{|c|c|c|c|c|c|c|c|c|}
\hline \multicolumn{9}{|c|}{ 2006-2008 } \\
\hline Model & $\begin{array}{c}\mathrm{N} \\
\text { Networks }\end{array}$ & Factor & Min & $\operatorname{Max}$ & q10 & q50 & q90 & $\begin{array}{c}\% \text { elements } \\
\leq-0.1\end{array}$ \\
\hline $\mathrm{Q}(10 \%)$ & 4 & $\mathrm{~N}$ & -0.465 & 0.611 & -0.191 & -0.001 & 0.201 & $25.6 \%$ \\
\hline $\mathrm{Q}(50 \%)$ & 4 & $\mathrm{~N}$ & -0.380 & 0.661 & -0.176 & 0.011 & 0.247 & $22.9 \%$ \\
\hline $\mathrm{Q}(90 \%)$ & 4 & $\mathrm{~N}$ & -0.443 & 0.608 & -0.195 & -0.006 & 0.206 & $25.9 \%$ \\
\hline $\mathrm{Q}(10 \%)$ & 4 & $\mathrm{Y}$ & -0.517 & 0.624 & -0.217 & -0.011 & 0.211 & $27.1 \%$ \\
\hline $\mathrm{Q}(50 \%)$ & 4 & $\mathrm{Y}$ & -0.467 & 0.690 & -0.199 & 0.006 & 0.237 & $25.2 \%$ \\
\hline $\mathrm{Q}(90 \%)$ & 4 & $\mathrm{Y}$ & -0.533 & 0.599 & -0.195 & -0.014 & 0.216 & $25.2 \%$ \\
\hline G & 1 & $\mathrm{~N}$ & -0.384 & 0.687 & -0.146 & 0.025 & 0.257 & $18.6 \%$ \\
\hline G & 1 & Y & -0.401 & 0.687 & -0.157 & 0.011 & 0.239 & $19.8 \%$ \\
\hline 4-F-CAPM & 0 & N.A & -0.486 & 0.733 & -0.219 & 0.003 & 0.267 & $27.1 \%$ \\
\hline \multicolumn{9}{|c|}{ 2011-2015 } \\
\hline Model & $\begin{array}{c}\mathrm{N} \\
\text { Networks }\end{array}$ & Factor & Min & $\operatorname{Max}$ & q10 & q50 & q90 & $\begin{array}{c}\% \text { elements } \\
\leq-0.1\end{array}$ \\
\hline $\mathrm{Q}(10 \%)$ & 4 & $\mathrm{~N}$ & -0.463 & 0.567 & -0.140 & -0.008 & 0.135 & $19.7 \%$ \\
\hline $\mathrm{Q}(50 \%)$ & 4 & $\mathrm{~N}$ & -0.447 & 0.519 & -0.138 & 0.007 & 0.176 & $16.6 \%$ \\
\hline $\mathrm{Q}(90 \%)$ & 4 & $\mathrm{~N}$ & -0.470 & 0.572 & -0.144 & -0.013 & 0.138 & $19.6 \%$ \\
\hline $\mathrm{Q}(10 \%)$ & 4 & $\mathrm{Y}$ & -0.442 & 0.501 & -0.148 & -0.003 & 0.145 & $19.3 \%$ \\
\hline $\mathrm{Q}(50 \%)$ & 4 & $\mathrm{Y}$ & -0.454 & 0.508 & -0.136 & 0.003 & 0.160 & $16.7 \%$ \\
\hline $\mathrm{Q}(90 \%)$ & 4 & $\mathrm{Y}$ & -0.443 & 0.567 & -0.144 & -0.005 & 0.153 & $18.4 \%$ \\
\hline G & 1 & $\mathrm{~N}$ & -0.470 & 0.577 & -0.140 & 0.007 & 0.199 & $18.0 \%$ \\
\hline $\mathrm{G}$ & 1 & $\mathrm{Y}$ & -0.503 & 0.577 & -0.139 & 0.006 & 0.177 & $17.7 \%$ \\
\hline 4-F-CAPM & 0 & N.A & -0.456 & 0.592 & -0.147 & 0.007 & 0.208 & $18.4 \%$ \\
\hline
\end{tabular}


Table F.19: The table report the $\delta$ of model (5) that represent the weights for networks combination. The top panel focused on the banks dataset, the middle panel on the insurance companies dataset and the bottom panel on the industry portfolios dataset. The first column identifies the quantiles used to estimate the quantile-based network, and the second column indicates if a common factor was used $(\mathrm{Y})$ or not used $(\mathrm{N})$ in the estimation of the causality networks. Columns 3 to 5 refer to the crisis sample while columns 6 to 8 to the most recent sample. The second row identifies the three different networks which are optimally combined: quantile-on-quantile causality Qo; non-parametric quantile causality - QN and Granger Causality. Parameters are, by construction, positive and sum up to one (within each row and within each period). A star identifies parameters significant at the $5 \%$ confidence level.

\begin{tabular}{|c|c|c|c|c|c|c|c|}
\hline & & \multicolumn{3}{|c|}{ 2006-2008 } & \multicolumn{3}{|c|}{$2010-2015$} \\
\hline Quantile & Factor & Qo & $\mathrm{QN}$ & GR & Qo & QN & GR \\
\hline \multicolumn{8}{|c|}{25 Banks } \\
\hline $10 \%$ & $\mathrm{~N}$ & 0.071 & $0.915^{*}$ & 0.014 & 0.630 & 0.354 & 0.017 \\
\hline $50 \%$ & $\mathrm{~N}$ & 0.128 & $0.848^{*}$ & 0.024 & 0.132 & $0.714^{*}$ & 0.154 \\
\hline $90 \%$ & $\mathrm{~N}$ & 0.008 & $0.884^{*}$ & 0.108 & $0.706^{*}$ & $0.243^{*}$ & $0.051^{*}$ \\
\hline $10 \%$ & $\mathrm{Y}$ & 0.082 & $0.843^{*}$ & 0.076 & 0.538 & $0.428^{*}$ & $0.034^{*}$ \\
\hline $50 \%$ & $\mathrm{Y}$ & 0.094 & 0.726 & 0.180 & 0.092 & $0.745^{*}$ & 0.163 \\
\hline $90 \%$ & $\mathrm{Y}$ & 0.002 & $0.893^{*}$ & 0.105 & $0.876^{*}$ & $0.121^{*}$ & $0.003^{*}$ \\
\hline \multicolumn{8}{|c|}{25 Insurance Companies } \\
\hline $10 \%$ & $\mathrm{~N}$ & 0.034 & $0.966^{*}$ & 0.000 & 0.804 & 0.141 & $0.055^{*}$ \\
\hline $50 \%$ & $\mathrm{~N}$ & 0.000 & $0.942^{*}$ & 0.058 & 0.054 & 0.615 & 0.331 \\
\hline $90 \%$ & $\mathrm{~N}$ & 0.028 & 0.972 & 0.000 & 0.364 & 0.436 & 0.200 \\
\hline $10 \%$ & $\mathrm{Y}$ & 0.006 & $0.949^{*}$ & 0.045 & 0.489 & 0.511 & 0.000 \\
\hline $50 \%$ & $\mathrm{Y}$ & 0.516 & 0.474 & 0.009 & 0.051 & $0.846^{*}$ & 0.102 \\
\hline $90 \%$ & $\mathrm{Y}$ & 0.046 & $0.954^{*}$ & 0.000 & 0.554 & 0.446 & $0.000^{*}$ \\
\hline \multicolumn{8}{|c|}{48 Industry Portfolio } \\
\hline $10 \%$ & $\mathrm{~N}$ & 0.173 & 0.057 & 0.770 & $1.000^{*}$ & 0.000 & $0.000^{*}$ \\
\hline $50 \%$ & $\mathrm{~N}$ & 0.079 & 0.248 & 0.673 & 0.030 & $0.868^{*}$ & 0.102 \\
\hline $90 \%$ & $\mathrm{~N}$ & $1.000^{*}$ & 0.000 & 0.000 & $1.000^{*}$ & 0.000 & $0.000^{*}$ \\
\hline $10 \%$ & $\mathrm{Y}$ & 0.619 & 0.052 & 0.329 & 0.640 & 0.320 & 0.039 \\
\hline $50 \%$ & $\mathrm{Y}$ & 0.000 & 0.676 & 0.324 & 0.029 & $0.876^{*}$ & 0.095 \\
\hline $90 \%$ & $\mathrm{Y}$ & 0.810 & $0.000^{*}$ & 0.190 & 0.918 & 0.032 & 0.050 \\
\hline
\end{tabular}


Table F.20: The table reports residual correlation descriptive analyses for the Banks dataset. The first column identify the various models, while the second column indicates the number of networks used in the model. In the first column $Q(10 \%)$ identifies the use of a combination of causality networks from quantile regression (Qo and QN) at the $10 \%$ quantile, combined with the Granger Causality Network. Similarly, when the reference quantile is $50 \%$ or $90 \%$. With $G$ we denote the model using just the Granger casuality network, while the last line refers to the 4-factor CAPM. The table reports statistics for the residuals correlations: the minimum, maximum, the $10 \%$ quantile $q_{10}$, the median $q_{50}$, the $90 \%$ quantile and the number of elements of the correlation matrix lower than -0.1 .

\begin{tabular}{|c|c|c|c|c|c|c|c|c|}
\hline \multicolumn{9}{|c|}{ 2006-2008 } \\
\hline Model & $\begin{array}{c}\text { N } \\
\text { Networks }\end{array}$ & Factor & Min & $\operatorname{Max}$ & q10 & q50 & q90 & $\begin{array}{c}\% \text { elements } \\
\leq-0.1\end{array}$ \\
\hline $\mathrm{Q}(10 \%)$ & 4 & $\mathrm{~N}$ & -0.304 & 0.584 & -0.117 & 0.067 & 0.273 & $12.0 \%$ \\
\hline $\mathrm{Q}(50 \%)$ & 4 & $\mathrm{~N}$ & -0.319 & 0.587 & -0.105 & 0.072 & 0.342 & $11.0 \%$ \\
\hline $\mathrm{Q}(90 \%)$ & 4 & $\mathrm{~N}$ & -0.269 & 0.600 & -0.147 & 0.050 & 0.278 & $16.0 \%$ \\
\hline $\mathrm{Q}(10 \%)$ & 4 & $\mathrm{Y}$ & -0.295 & 0.571 & -0.136 & 0.035 & 0.236 & $15.3 \%$ \\
\hline $\mathrm{Q}(50 \%)$ & 4 & $\mathrm{Y}$ & -0.346 & 0.490 & -0.119 & 0.048 & 0.288 & $12.3 \%$ \\
\hline $\mathrm{Q}(90 \%)$ & 4 & $\mathrm{Y}$ & -0.313 & 0.485 & -0.142 & 0.031 & 0.244 & $18.0 \%$ \\
\hline G & 1 & $\mathrm{~N}$ & -0.381 & 0.670 & -0.128 & 0.111 & 0.372 & $13.3 \%$ \\
\hline $\mathrm{G}$ & 1 & $\mathrm{Y}$ & -0.394 & 0.641 & -0.128 & 0.089 & 0.378 & $11.7 \%$ \\
\hline 4-F-CAPM & 0 & N.A & -0.358 & 0.678 & -0.108 & 0.257 & 0.502 & $10.7 \%$ \\
\hline \multicolumn{9}{|c|}{ 2011-2015 } \\
\hline Model & $\begin{array}{c}\mathrm{N} \\
\text { Networks }\end{array}$ & Factor & Min & $\operatorname{Max}$ & q10 & q50 & q90 & $\begin{array}{c}\% \text { elements } \\
\leq-0.1\end{array}$ \\
\hline $\mathrm{Q}(10 \%)$ & 4 & $\mathrm{~N}$ & -0.263 & 0.422 & -0.122 & -0.013 & 0.135 & $15.3 \%$ \\
\hline $\mathrm{Q}(50 \%)$ & 4 & $\mathrm{~N}$ & -0.332 & 0.528 & -0.116 & 0.052 & 0.243 & $12.7 \%$ \\
\hline $\mathrm{Q}(90 \%)$ & 4 & $\mathrm{~N}$ & -0.253 & 0.431 & -0.127 & 0.010 & 0.138 & $15.7 \%$ \\
\hline $\mathrm{Q}(10 \%)$ & 4 & $\mathrm{Y}$ & -0.283 & 0.503 & -0.132 & -0.008 & 0.140 & $19.0 \%$ \\
\hline $\mathrm{Q}(50 \%)$ & 4 & $\mathrm{Y}$ & -0.311 & 0.522 & -0.122 & 0.052 & 0.237 & $13.0 \%$ \\
\hline $\mathrm{Q}(90 \%)$ & 4 & $\mathrm{Y}$ & -0.304 & 0.508 & -0.140 & 0.001 & 0.132 & $17.7 \%$ \\
\hline $\mathrm{G}$ & 1 & $\mathrm{~N}$ & -0.211 & 0.598 & -0.027 & 0.151 & 0.362 & $3.0 \%$ \\
\hline $\mathrm{G}$ & 1 & $\mathrm{Y}$ & -0.211 & 0.598 & -0.027 & 0.146 & 0.361 & $3.3 \%$ \\
\hline 4-F-CAPM & 0 & NA & -0.122 & 0.669 & 0.054 & 0.266 & 0.487 & $0.3 \%$ \\
\hline
\end{tabular}


Table F.21: The table reports residual correlation descriptive analyses for the Insurance Companies dataset. The first column identify the various models, while the second column indicates the number of networks used in the model. In the first column $Q(10 \%)$ identifies the use of a combination of causality networks from quantile regression (Qo and QN) at the $10 \%$ quantile, combined with the Granger Causality Network. Similarly, when the reference quantile is 50\% or $90 \%$. With $G$ we denote the model using just the Granger casuality network, while the last line refers to the 4-factor CAPM. The table reports statistics for the residuals correlations: the minimum, maximum, the $10 \%$ quantile $q_{10}$, the median $q_{50}$, the $90 \%$ quantile and the number of elements of the correlation matrix lower than -0.1 .

\begin{tabular}{|c|c|c|c|c|c|c|c|c|}
\hline \multicolumn{9}{|c|}{ 2006-2008 } \\
\hline Model & $\begin{array}{c}\mathbf{N} \\
\text { Networks }\end{array}$ & Factor & Min & $\operatorname{Max}$ & $q 10$ & q50 & q90 & $\begin{array}{c}\% \text { elements } \\
\leq-0.1\end{array}$ \\
\hline $\mathrm{Q}(10 \%)$ & 4 & $\mathrm{~N}$ & -0.441 & 0.682 & -0.222 & 0.002 & 0.248 & $27.0 \%$ \\
\hline $\mathrm{Q}(50 \%)$ & 4 & $\mathrm{~N}$ & -0.435 & 0.756 & -0.169 & 0.027 & 0.335 & $20.7 \%$ \\
\hline $\mathrm{Q}(90 \%)$ & 4 & $\mathrm{~N}$ & -0.448 & 0.676 & -0.207 & 0.009 & 0.255 & $25.7 \%$ \\
\hline $\mathrm{Q}(10 \%)$ & 4 & $\mathrm{Y}$ & -0.436 & 0.684 & -0.203 & 0.005 & 0.246 & $28.0 \%$ \\
\hline $\mathrm{Q}(50 \%)$ & 4 & $\mathrm{Y}$ & -0.468 & 0.574 & -0.185 & 0.016 & 0.238 & $22.7 \%$ \\
\hline $\mathrm{Q}(90 \%)$ & 4 & $\mathrm{Y}$ & -0.464 & 0.671 & -0.214 & 0.001 & 0.253 & $27.0 \%$ \\
\hline G & 1 & $\mathrm{~N}$ & -0.466 & 0.831 & -0.166 & 0.044 & 0.316 & $17.7 \%$ \\
\hline $\mathrm{G}$ & 1 & $\mathrm{Y}$ & -0.460 & 0.714 & -0.166 & 0.048 & 0.323 & $15.0 \%$ \\
\hline 4-F-CAPM & 0 & N.A & -0.370 & 0.847 & -0.149 & 0.089 & 0.422 & $14.0 \%$ \\
\hline \multicolumn{9}{|c|}{ 2011-2015 } \\
\hline Model & $\begin{array}{c}\mathrm{N} \\
\text { Networks }\end{array}$ & Factor & Min & $\operatorname{Max}$ & q10 & q50 & q90 & $\begin{array}{c}\% \text { elements } \\
\leq-0.1\end{array}$ \\
\hline $\mathrm{Q}(10 \%)$ & 4 & $\mathrm{~N}$ & -0.293 & 0.482 & -0.123 & 0.009 & 0.158 & $14.7 \%$ \\
\hline $\mathrm{Q}(50 \%)$ & 4 & $\mathrm{~N}$ & -0.238 & 0.593 & -0.132 & 0.025 & 0.217 & $15.0 \%$ \\
\hline $\mathrm{Q}(90 \%)$ & 4 & $\mathrm{~N}$ & -0.289 & 0.348 & -0.137 & -0.006 & 0.167 & $19.3 \%$ \\
\hline $\mathrm{Q}(10 \%)$ & 4 & $\mathrm{Y}$ & -0.333 & 0.503 & -0.153 & -0.012 & 0.189 & $20.7 \%$ \\
\hline $\mathrm{Q}(50 \%)$ & 4 & $\mathrm{Y}$ & -0.276 & 0.590 & -0.140 & 0.020 & 0.210 & $17.0 \%$ \\
\hline $\mathrm{Q}(90 \%)$ & 4 & $\mathrm{Y}$ & -0.299 & 0.398 & -0.152 & -0.001 & 0.199 & $21.3 \%$ \\
\hline G & 1 & $\mathrm{~N}$ & -0.136 & 0.658 & -0.005 & 0.121 & 0.317 & $1.7 \%$ \\
\hline $\mathrm{G}$ & 1 & $\mathrm{Y}$ & -0.136 & 0.658 & -0.014 & 0.116 & 0.315 & $1.7 \%$ \\
\hline 4-F-CAPM & 0 & N.A & -0.103 & 0.658 & 0.017 & 0.138 & 0.332 & $0.3 \%$ \\
\hline
\end{tabular}


Table F.22: The table reports residual correlation descriptive analyses for the Industry portfolios dataset. The first column identify the various models, while the second column indicates the number of networks used in the model. In the first column $Q(10 \%)$ identifies the use of a combination of causality networks from quantile regression (Qo and QN) at the 10\% quantile, combined with the Granger Causality Network. Similarly, when the reference quantile is 50\% or $90 \%$. With $G$ we denote the model using just the Granger casuality network, while the last line refers to the 4-factor CAPM. The table reports statistics for the residuals correlations: the minimum, maximum, the $10 \%$ quantile $q_{10}$, the median $q_{50}$, the $90 \%$ quantile and the number of elements of the correlation matrix lower than -0.1 .

2006-2008

\begin{tabular}{ccccccccc}
\hline Model & $\begin{array}{c}\text { N } \\
\text { Networks }\end{array}$ & Factor & Min & Max & q10 & q50 & q90 & $\begin{array}{c}\text { \% elements } \\
\leq-0.1\end{array}$ \\
\hline Q(10\%) & 4 & N & -0.370 & 0.592 & -0.153 & 0.013 & 0.237 & $20.0 \%$ \\
Q(50\%) & 4 & N & -0.357 & 0.604 & -0.151 & 0.020 & 0.251 & $19.3 \%$ \\
Q(90\%) & 4 & N & -0.465 & 0.660 & -0.196 & -0.002 & 0.212 & $25.8 \%$ \\
Q(10\%) & 4 & Y & -0.431 & 0.595 & -0.182 & 0.007 & 0.221 & $22.5 \%$ \\
Q(50\%) & 4 & Y & -0.415 & 0.687 & -0.169 & 0.006 & 0.231 & $21.5 \%$ \\
Q(90\%) & 4 & Y & -0.508 & 0.592 & -0.187 & -0.007 & 0.215 & $23.8 \%$ \\
G & 1 & N & -0.384 & 0.687 & -0.146 & 0.025 & 0.257 & $18.6 \%$ \\
G & 1 & Y & -0.401 & 0.687 & -0.157 & 0.011 & 0.239 & $19.8 \%$ \\
$4-F-C A P M$ & 0 & N.A & -0.486 & 0.733 & -0.219 & 0.003 & 0.267 & $27.1 \%$ \\
& & & $\mathbf{2 0 1 1 - 2 0 1 5}$ & & & & \\
\hline Model & N & Factor & Min & Max & $\mathbf{q 1 0}$ & $\mathbf{q 5 0}$ & $\mathbf{q 9 0}$ & $\mathbf{\%}$ elements \\
& Networks & & & & & & & $\leq-0.1$ \\
\hline Q(10\%) & 4 & N & -0.463 & 0.567 & -0.138 & -0.008 & 0.133 & $19.3 \%$ \\
Q(50\%) & 4 & N & -0.464 & 0.519 & -0.137 & 0.003 & 0.172 & $16.8 \%$ \\
Q(90\%) & 4 & N & -0.471 & 0.572 & -0.144 & -0.013 & 0.139 & $20.0 \%$ \\
Q(10\%) & 4 & Y & -0.434 & 0.495 & -0.148 & -0.005 & 0.145 & $19.1 \%$ \\
Q(50\%) & 4 & Y & -0.509 & 0.513 & -0.141 & 0.004 & 0.163 & $16.5 \%$ \\
Q(90\%) & 4 & Y & -0.424 & 0.563 & -0.144 & -0.004 & 0.150 & $19.3 \%$ \\
G & 1 & N & -0.470 & 0.577 & -0.140 & 0.007 & 0.199 & $18.0 \%$ \\
G & 1 & Y & -0.503 & 0.577 & -0.139 & 0.006 & 0.177 & $17.7 \%$ \\
\hline
\end{tabular}




\section{G Tables for Monthly returns}

Table G.23: The table reports the $\delta$ of model (5) that represent the weights for networks combination. The top panel focused on the banks dataset, the middle panel on the insurance companies dataset and the bottom panel on the industry portfolios dataset. The first column identifies the quantiles used to estimate the quantile-based network, and the second column indicates if a common factor was used $(\mathrm{Y})$ or not used $(\mathrm{N})$ in the estimation of the causality networks. Columns 3 to 6 refer to the crisis sample while columns 7 to 10 to the most recent sample. The second row identifies the four different networks which are optimally combined: baseline quantile causality - QB; quantile-on-quantile causality Qo; non-parametric quantile causality - QN; Granger causality. Parameters are, by construction, positive and sum up to one (within each row and within each period). A star identifies parameters significant at the $5 \%$ confidence level.

\begin{tabular}{|c|c|c|c|c|c|c|c|c|c|}
\hline \multirow[b]{2}{*}{ Quantile } & \multirow[b]{2}{*}{ Factor } & \multicolumn{4}{|c|}{ 2006-2008 } & \multicolumn{4}{|c|}{ 2010-2015 } \\
\hline & & QB & Qo & QN & QR & QB & Qo & QN & QR \\
\hline \multicolumn{10}{|c|}{25 Banks } \\
\hline $10 \%$ & $\mathrm{~N}$ & 0.031 & 0.091 & 0.878 & 0.000 & 0.000 & $0.692^{*}$ & 0.271 & 0.037 \\
\hline $50 \%$ & $\mathrm{~N}$ & 0.495 & $0.000^{*}$ & 0.345 & 0.159 & 0.083 & $0.123^{*}$ & $0.689^{*}$ & 0.105 \\
\hline $90 \%$ & $\mathrm{~N}$ & 0.002 & 0.007 & $0.872^{*}$ & 0.119 & 0.000 & $0.739^{*}$ & 0.142 & 0.119 \\
\hline $10 \%$ & $\mathrm{Y}$ & 0.061 & 0.182 & $0.681^{*}$ & 0.077 & 0.000 & 0.604 & 0.375 & 0.022 \\
\hline $50 \%$ & $\mathrm{Y}$ & 0.131 & $0.109^{*}$ & $0.451^{*}$ & $0.310^{*}$ & 0.075 & $0.046^{*}$ & $0.746^{*}$ & 0.132 \\
\hline $90 \%$ & $\mathrm{Y}$ & 0.131 & 0.022 & $0.639^{*}$ & 0.207 & 0.000 & $0.954^{*}$ & 0.000 & 0.046 \\
\hline \multicolumn{10}{|c|}{25 Insurance Companies } \\
\hline $10 \%$ & $\mathrm{~N}$ & 0.248 & $0.000^{*}$ & $0.638^{*}$ & 0.115 & 0.000 & 0.651 & 0.137 & 0.213 \\
\hline $50 \%$ & $\mathrm{~N}$ & 0.689 & 0.000 & $0.175^{*}$ & 0.136 & 0.052 & 0.063 & 0.637 & 0.248 \\
\hline $90 \%$ & $\mathrm{~N}$ & 0.000 & $0.042^{*}$ & $0.829^{*}$ & 0.129 & 0.000 & 0.449 & 0.453 & 0.098 \\
\hline $10 \%$ & $\mathrm{Y}$ & 0.000 & $0.005^{*}$ & $0.916^{*}$ & 0.079 & 0.000 & $1.000^{*}$ & 0.000 & 0.000 \\
\hline $50 \%$ & $\mathrm{Y}$ & 0.000 & $0.071^{*}$ & $0.831^{*}$ & 0.098 & 0.082 & 0.054 & 0.862 & 0.003 \\
\hline $90 \%$ & $\mathrm{Y}$ & 0.000 & 0.000 & 0.907 & 0.093 & 0.000 & 0.186 & $0.814^{*}$ & 0.000 \\
\hline \multicolumn{10}{|c|}{48 Industry Portfolio } \\
\hline $10 \%$ & $\mathrm{~N}$ & 0.149 & 0.253 & 0.599 & 0.000 & 0.020 & 0.980 & 0.000 & 0.000 \\
\hline $50 \%$ & $\mathrm{~N}$ & 0.085 & 0.119 & 0.000 & 0.797 & 0.309 & 0.031 & 0.520 & 0.141 \\
\hline $90 \%$ & $\mathrm{~N}$ & 0.140 & 0.000 & 0.860 & 0.000 & 0.014 & 0.203 & $0.633^{*}$ & 0.150 \\
\hline $10 \%$ & $\mathrm{Y}$ & 0.304 & 0.226 & $0.356^{*}$ & 0.114 & 0.012 & 0.576 & 0.294 & 0.118 \\
\hline $50 \%$ & $\mathrm{Y}$ & 0.426 & 0.137 & 0.000 & 0.437 & 0.093 & 0.027 & $0.767^{*}$ & 0.113 \\
\hline $90 \%$ & $\mathrm{Y}$ & 0.166 & 0.030 & 0.749 & 0.055 & 0.103 & 0.668 & 0.106 & 0.123 \\
\hline
\end{tabular}


Table G.24: The table reports residual correlation descriptive analyses for the Banks dataset. The first column identify the various models, while the second column indicates the number of networks used in the model. In the first column $Q(10 \%)$ identifies the use of a combination of causality networks from quantile regression (QB, Qo and QN) at the $10 \%$ quantile, combined with the Granger causality network. Similarly, when the reference quantile is $50 \%$ or $90 \%$. With $G$ we denote the model using just the Granger casuality network, while the last line refers to the 4-factor CAPM. The table reports statistics for the residuals correlations: the minimum, maximum, the $10 \%$ quantile $q_{10}$, the median $q_{50}$, the $90 \%$ quantile and the number of elements of the correlation matrix lower than -0.1 .

Table G.25: Add caption

\begin{tabular}{|c|c|c|c|c|c|c|c|c|}
\hline \multicolumn{9}{|c|}{ 2006-2008 } \\
\hline Model & $\begin{array}{c}\mathrm{N} \\
\text { Networks }\end{array}$ & Factor & Min & Max & q10 & q50 & q90 & $\begin{array}{c}\% \text { elements } \\
\leq-0.1\end{array}$ \\
\hline $\mathrm{Q}(10 \%)$ & 4 & $\mathrm{~N}$ & -0.485 & 0.748 & -0.229 & 0.062 & 0.358 & $23.7 \%$ \\
\hline $\mathrm{Q}(50 \%)$ & 4 & $\mathrm{~N}$ & -0.401 & 0.736 & -0.193 & 0.095 & 0.412 & $20.7 \%$ \\
\hline $\mathrm{Q}(90 \%)$ & 4 & $\mathrm{~N}$ & -0.496 & 0.696 & -0.236 & 0.071 & 0.367 & $26.3 \%$ \\
\hline $\mathrm{Q}(10 \%)$ & 4 & $\mathrm{Y}$ & -0.499 & 0.797 & -0.248 & 0.034 & 0.325 & $30.3 \%$ \\
\hline $\mathrm{Q}(50 \%)$ & 4 & $\mathrm{Y}$ & -0.495 & 0.755 & -0.226 & 0.055 & 0.351 & $26.3 \%$ \\
\hline $\mathrm{Q}(90 \%)$ & 4 & $\mathrm{Y}$ & -0.504 & 0.737 & -0.257 & 0.050 & 0.323 & $28.3 \%$ \\
\hline $\mathrm{G}$ & 1 & $\mathrm{~N}$ & -0.605 & 0.756 & -0.172 & 0.190 & 0.528 & $17.0 \%$ \\
\hline $\mathrm{G}$ & 1 & $\mathrm{Y}$ & -0.599 & 0.826 & -0.190 & 0.141 & 0.505 & $18.0 \%$ \\
\hline 4-F-CAPM & 0 & N.A & -0.404 & 0.826 & 0.040 & 0.417 & 0.699 & $7.0 \%$ \\
\hline \multicolumn{9}{|c|}{ 2011-2015 } \\
\hline Model & $\begin{array}{c}\mathrm{N} \\
\text { Networks }\end{array}$ & Factor & Min & $\operatorname{Max}$ & q10 & q50 & q90 & $\begin{array}{c}\% \text { elements } \\
\leq-0.1\end{array}$ \\
\hline $\mathrm{Q}(10 \%)$ & 4 & $\mathrm{~N}$ & -0.415 & 0.527 & -0.209 & -0.007 & 0.209 & $28.0 \%$ \\
\hline $\mathrm{Q}(50 \%)$ & 4 & $\mathrm{~N}$ & -0.536 & 0.602 & -0.189 & 0.033 & 0.288 & $21.3 \%$ \\
\hline $\mathrm{Q}(90 \%)$ & 4 & $\mathrm{~N}$ & -0.455 & 0.512 & -0.200 & 0.007 & 0.227 & $27.7 \%$ \\
\hline $\mathrm{Q}(10 \%)$ & 4 & $\mathrm{Y}$ & -0.382 & 0.563 & -0.226 & -0.011 & 0.228 & $28.0 \%$ \\
\hline $\mathrm{Q}(50 \%)$ & 4 & $\mathrm{Y}$ & -0.449 & 0.582 & -0.201 & 0.038 & 0.280 & $22.7 \%$ \\
\hline $\mathrm{Q}(90 \%)$ & 4 & $\mathrm{Y}$ & -0.423 & 0.564 & -0.223 & -0.003 & 0.224 & $27.7 \%$ \\
\hline G & 1 & $\mathrm{~N}$ & -0.314 & 0.677 & -0.083 & 0.165 & 0.460 & $8.3 \%$ \\
\hline $\mathrm{G}$ & 1 & $\mathrm{Y}$ & -0.388 & 0.677 & -0.083 & 0.162 & 0.455 & $8.3 \%$ \\
\hline 4-F-CAPM & 0 & NA & -0.155 & 0.793 & 0.098 & 0.385 & 0.604 & $0.7 \%$ \\
\hline
\end{tabular}


Table G.26: The table reports residual correlation descriptive analyses for the Insurance Companies dataset. The first column identify the various models, while the second column indicates the number of networks used in the model. In the first column $Q(10 \%)$ identifies the use of a combination of causality networks from quantile regression (QB, Qo and QN) at the $10 \%$ quantile, combined with the Granger causality network. Similarly, when the reference quantile is $50 \%$ or $90 \%$. With $G$ we denote the model using just the Granger casuality network, while the last line refers to the 4-factor CAPM. The table reports statistics for the residuals correlations: the minimum, maximum, the $10 \%$ quantile $q_{10}$, the median $q_{50}$, the $90 \%$ quantile and the number of elements of the correlation matrix lower than -0.1 .

\begin{tabular}{|c|c|c|c|c|c|c|c|c|}
\hline \multicolumn{9}{|c|}{ 2006-2008 } \\
\hline Model & $\begin{array}{c}\mathbf{N} \\
\text { Networks }\end{array}$ & Factor & Min & $\operatorname{Max}$ & q10 & q50 & q90 & $\begin{array}{c}\% \text { elements } \\
\leq-0.1\end{array}$ \\
\hline $\mathrm{Q}(10 \%)$ & 4 & $\mathrm{~N}$ & -0.684 & 0.693 & -0.264 & 0.040 & 0.372 & $26.3 \%$ \\
\hline $\mathrm{Q}(50 \%)$ & 4 & $\mathrm{~N}$ & -0.674 & 0.760 & -0.261 & 0.039 & 0.366 & $26.0 \%$ \\
\hline $\mathrm{Q}(90 \%)$ & 4 & $\mathrm{~N}$ & -0.694 & 0.744 & -0.276 & 0.044 & 0.367 & $27.3 \%$ \\
\hline $\mathrm{Q}(10 \%)$ & 4 & $\mathrm{Y}$ & -0.674 & 0.734 & -0.277 & 0.054 & 0.383 & $29.0 \%$ \\
\hline $\mathrm{Q}(50 \%)$ & 4 & $\mathrm{Y}$ & -0.694 & 0.755 & -0.271 & 0.041 & 0.370 & $28.7 \%$ \\
\hline $\mathrm{Q}(90 \%)$ & 4 & $\mathrm{Y}$ & -0.679 & 0.734 & -0.270 & 0.055 & 0.383 & $28.3 \%$ \\
\hline G & 1 & $\mathrm{~N}$ & -0.665 & 0.771 & -0.267 & 0.055 & 0.399 & $27.7 \%$ \\
\hline $\mathrm{G}$ & 1 & $\mathrm{Y}$ & -0.629 & 0.771 & -0.240 & 0.065 & 0.398 & $24.7 \%$ \\
\hline 4-F-CAPM & 0 & N.A & -0.639 & 0.886 & -0.190 & 0.157 & 0.519 & $16.7 \%$ \\
\hline \multicolumn{9}{|c|}{ 2011-2015 } \\
\hline Model & $\begin{array}{c}\mathrm{N} \\
\text { Networks }\end{array}$ & Factor & Min & $\operatorname{Max}$ & q10 & q50 & q90 & $\begin{array}{c}\% \text { elements } \\
\leq-0.1\end{array}$ \\
\hline $\mathrm{Q}(10 \%)$ & 4 & $\mathrm{~N}$ & -0.543 & 0.610 & -0.206 & 0.010 & 0.236 & $26.0 \%$ \\
\hline $\mathrm{Q}(50 \%)$ & 4 & $\mathrm{~N}$ & -0.428 & 0.648 & -0.224 & 0.037 & 0.283 & $24.3 \%$ \\
\hline $\mathrm{Q}(90 \%)$ & 4 & $\mathrm{~N}$ & -0.450 & 0.577 & -0.229 & 0.001 & 0.247 & $27.7 \%$ \\
\hline $\mathrm{Q}(10 \%)$ & 4 & $\mathrm{Y}$ & -0.533 & 0.629 & -0.239 & 0.008 & 0.264 & $31.7 \%$ \\
\hline $\mathrm{Q}(50 \%)$ & 4 & $\mathrm{Y}$ & -0.442 & 0.697 & -0.236 & 0.036 & 0.287 & $26.7 \%$ \\
\hline $\mathrm{Q}(90 \%)$ & 4 & $\mathrm{Y}$ & -0.446 & 0.626 & -0.242 & 0.002 & 0.280 & $31.0 \%$ \\
\hline G & 1 & $\mathrm{~N}$ & -0.230 & 0.826 & -0.015 & 0.221 & 0.473 & $2.3 \%$ \\
\hline $\mathrm{G}$ & 1 & $\mathrm{Y}$ & -0.230 & 0.826 & -0.023 & 0.218 & 0.473 & $2.7 \%$ \\
\hline 4-F-CAPM & 0 & N.A & -0.204 & 0.826 & 0.045 & 0.246 & 0.475 & $0.7 \%$ \\
\hline
\end{tabular}


Table G.27: The table reports residual correlation descriptive analyses for the Industry portfolios dataset. The first column identify the various models, while the second column indicates the number of networks used in the model. In the first column $Q(10 \%)$ identifies the use of a combination of causality networks from quantile regression (QB, Qo and QN) at the $10 \%$ quantile, combined with the Granger causality network. Similarly, when the reference quantile is 50\% or $90 \%$. With $G$ we denote the model using just the Granger casuality network, while the last line refers to the 4-factor CAPM. The table reports statistics for the residuals correlations: the minimum, maximum, the $10 \%$ quantile $q_{10}$, the median $q_{50}$, the $90 \%$ quantile and the number of elements of the correlation matrix lower than -0.1 .

\begin{tabular}{|c|c|c|c|c|c|c|c|c|}
\hline \multicolumn{9}{|c|}{ 2006-2008 } \\
\hline Model & $\begin{array}{c}\mathrm{N} \\
\text { Networks }\end{array}$ & Factor & Min & $\operatorname{Max}$ & q10 & q50 & q90 & $\begin{array}{c}\% \text { elements } \\
\backslash \text { le }-0.1\end{array}$ \\
\hline $\mathrm{Q}(10 \%)$ & 4 & $\mathrm{~N}$ & -0.702 & 0.653 & -0.338 & -0.029 & 0.267 & $39.0 \%$ \\
\hline $\mathrm{Q}(50 \%)$ & 4 & $\mathrm{~N}$ & -0.654 & 0.680 & -0.353 & -0.032 & 0.296 & $39.7 \%$ \\
\hline $\mathrm{Q}(90 \%)$ & 4 & $\mathrm{~N}$ & -0.619 & 0.658 & -0.347 & -0.027 & 0.289 & $38.7 \%$ \\
\hline $\mathrm{Q}(10 \%)$ & 4 & $\mathrm{Y}$ & -0.749 & 0.638 & -0.319 & -0.022 & 0.265 & $37.3 \%$ \\
\hline $\mathrm{Q}(50 \%)$ & 4 & $\mathrm{Y}$ & -0.675 & 0.675 & -0.371 & -0.031 & 0.318 & $40.8 \%$ \\
\hline $\mathrm{Q}(90 \%)$ & 4 & $\mathrm{Y}$ & -0.690 & 0.689 & -0.348 & -0.030 & 0.277 & $39.5 \%$ \\
\hline G & 1 & $\mathrm{~N}$ & -0.654 & 0.685 & -0.368 & -0.023 & 0.293 & $39.2 \%$ \\
\hline $\mathrm{G}$ & 1 & $\mathrm{Y}$ & -0.695 & 0.705 & -0.373 & -0.020 & 0.301 & $40.3 \%$ \\
\hline 4-F-CAPM & 0 & N.A & -0.667 & 0.713 & -0.378 & -0.025 & 0.309 & $39.5 \%$ \\
\hline \multicolumn{9}{|c|}{ 2011-2015 } \\
\hline Model & $\begin{array}{c}\mathrm{N} \\
\text { Networks }\end{array}$ & Factor & Min & $\operatorname{Max}$ & q10 & q50 & q90 & $\begin{array}{c}\% \text { elements } \\
\leq-0.1\end{array}$ \\
\hline $\mathrm{Q}(10 \%)$ & 4 & $\mathrm{~N}$ & -0.583 & 0.623 & -0.234 & -0.008 & 0.210 & $30.4 \%$ \\
\hline $\mathrm{Q}(50 \%)$ & 4 & $\mathrm{~N}$ & -0.499 & 0.617 & -0.206 & 0.014 & 0.248 & $26.1 \%$ \\
\hline $\mathrm{Q}(90 \%)$ & 4 & $\mathrm{~N}$ & -0.551 & 0.589 & -0.224 & -0.006 & 0.244 & $29.1 \%$ \\
\hline $\mathrm{Q}(10 \%)$ & 4 & $\mathrm{Y}$ & -0.534 & 0.543 & -0.228 & -0.002 & 0.244 & $29.5 \%$ \\
\hline $\mathrm{Q}(50 \%)$ & 4 & $\mathrm{Y}$ & -0.443 & 0.529 & -0.216 & 0.008 & 0.241 & $25.3 \%$ \\
\hline $\mathrm{Q}(90 \%)$ & 4 & $\mathrm{Y}$ & -0.446 & 0.585 & -0.230 & -0.002 & 0.242 & $29.5 \%$ \\
\hline G & 1 & $\mathrm{~N}$ & -0.502 & 0.579 & -0.228 & 0.009 & 0.259 & $28.0 \%$ \\
\hline $\mathrm{G}$ & 1 & $\mathrm{Y}$ & -0.478 & 0.594 & -0.220 & 0.010 & 0.251 & $27.7 \%$ \\
\hline 4-F-CAPM & 0 & N.A & -0.502 & 0.626 & -0.231 & 0.021 & 0.283 & $28.4 \%$ \\
\hline
\end{tabular}




\section{H Homogeneity}

Table H.28: The table reports the $\delta$ of model (5), that represent the weights for networks combination. The model replaces the matrix $\mathcal{R}$ with a scalar coefficient, and thus we have, across assets, a homogeneous impact of the network. The top panel focused on the banks dataset, the middle panel on the insurance companies dataset and the bottom panel on the industry portfolios dataset. The first column identifies the quantiles used to estimate the quantile-based network, and the second column indicates if a common factor was used $(\mathrm{Y})$ or not used $(\mathrm{N})$ in the estimation of the causality networks. Columns 3 to 6 refer to the crisis sample while columns 7 to 10 to the most recent sample. The second row identifies the four different networks which are optimally combined: baseline quantile causality - QB; quantile-on-quantile causality Qo; non-parametric quantile causality - QN; Granger causality. Parameters are, by construction, positive and sum up to one (within each row and within each period). A star identifies parameters significant at the $5 \%$ confidence level.

\begin{tabular}{|c|c|c|c|c|c|c|c|c|c|}
\hline \multirow[b]{2}{*}{ Quantile } & \multirow[b]{2}{*}{ Factor } & \multicolumn{4}{|c|}{ 2006-2008 } & \multicolumn{4}{|c|}{$2010-2015$} \\
\hline & & QB & Qo & QN & QG & QB & Qo & $\mathrm{QN}$ & QG \\
\hline \multicolumn{10}{|c|}{25 Banks } \\
\hline $10 \%$ & $\mathrm{~N}$ & 0.000 & 0.159 & $0.841^{*}$ & 0.000 & 0.028 & $0.656^{*}$ & $0.264^{*}$ & 0.052 \\
\hline $50 \%$ & $\mathrm{~N}$ & 0.199 & $0.146^{*}$ & $0.589^{*}$ & $0.066^{*}$ & $0.140^{*}$ & $0.273^{*}$ & $0.349^{*}$ & $0.238^{*}$ \\
\hline $90 \%$ & $\mathrm{~N}$ & 0.001 & 0.000 & $0.984^{*}$ & 0.000 & 0.048 & $0.565^{*}$ & $0.281^{*}$ & 0.106 \\
\hline $10 \%$ & $\mathrm{Y}$ & $0.000^{*}$ & $0.398^{*}$ & $0.403^{*}$ & $0.199^{*}$ & 0.036 & $0.771^{*}$ & 0.160 & $0.033^{*}$ \\
\hline $50 \%$ & $\mathrm{Y}$ & $0.140^{*}$ & $0.204^{*}$ & $0.444^{*}$ & $0.212^{*}$ & $0.198^{*}$ & $0.187^{*}$ & $0.409^{*}$ & $0.206^{*}$ \\
\hline $90 \%$ & $\mathrm{Y}$ & $0.247^{*}$ & $0.000^{*}$ & $0.471^{*}$ & $0.282^{*}$ & 0.000 & $0.939 *$ & $0.061^{*}$ & $0.000^{*}$ \\
\hline \multicolumn{10}{|c|}{25 Insurance Companies } \\
\hline $10 \%$ & $\mathrm{~N}$ & 0.061 & $0.151^{*}$ & $0.789^{*}$ & $0.000^{*}$ & 0.078 & $0.922^{*}$ & 0.000 & 0.000 \\
\hline $50 \%$ & $\mathrm{~N}$ & 0.193 & $0.099^{*}$ & $0.495^{*}$ & $0.212^{*}$ & 0.158 & $0.111^{*}$ & $0.659^{*}$ & $0.072^{*}$ \\
\hline $90 \%$ & $\mathrm{~N}$ & $0.000^{*}$ & $0.000^{*}$ & $1.000^{*}$ & $0.000^{*}$ & $0.000^{*}$ & $0.492^{*}$ & $0.508^{*}$ & $0.000^{*}$ \\
\hline $10 \%$ & $\mathrm{Y}$ & 0.040 & 0.082 & $0.856^{*}$ & 0.022 & 0.007 & $0.645^{*}$ & $0.347^{*}$ & 0.000 \\
\hline $50 \%$ & $\mathrm{Y}$ & 0.090 & $0.218^{*}$ & $0.631^{*}$ & 0.060 & 0.038 & 0.072 & $0.831^{*}$ & 0.058 \\
\hline $90 \%$ & $\mathrm{Y}$ & 0.000 & 0.000 & $0.973^{*}$ & 0.027 & $0.000^{*}$ & $0.566^{*}$ & $0.434^{*}$ & $0.000^{*}$ \\
\hline \multicolumn{10}{|c|}{48 Industry Portfolio } \\
\hline $10 \%$ & $\mathrm{~N}$ & $0.000^{*}$ & $0.000^{*}$ & $0.000^{*}$ & $1.000^{*}$ & 0.074 & $0.926^{*}$ & $0.000^{*}$ & $0.000^{*}$ \\
\hline $50 \%$ & $\mathrm{~N}$ & 0.000 & 0.213 & 0.177 & 0.610 & $0.000^{*}$ & $1.000^{*}$ & $0.000 *$ & $0.000^{*}$ \\
\hline $90 \%$ & $\mathrm{~N}$ & $0.000^{*}$ & $0.000^{*}$ & $0.000^{*}$ & $1.000^{*}$ & $0.000^{*}$ & $1.000^{*}$ & $0.000^{*}$ & $0.000^{*}$ \\
\hline $10 \%$ & $\mathrm{Y}$ & $1.000 *$ & $0.000^{*}$ & $0.000^{*}$ & $0.000^{*}$ & $0.000^{*}$ & $1.000^{*}$ & $0.000^{*}$ & $0.000^{*}$ \\
\hline $50 \%$ & $\mathrm{Y}$ & $1.000^{*}$ & $0.000^{*}$ & $0.000^{*}$ & $0.000^{*}$ & 0.705 & $0.001^{*}$ & $0.294^{*}$ & $0.000^{*}$ \\
\hline $90 \%$ & $\mathrm{Y}$ & $0.782^{*}$ & $0.218^{*}$ & $0.000 *$ & $0.000^{*}$ & $0.000^{*}$ & $1.000^{*}$ & $0.000 *$ & $0.000^{*}$ \\
\hline
\end{tabular}


Table H.29: The table reports scalar coefficient $\rho$ replacing matrix $\mathcal{R}$ in model (5), to impose homogeneous reaction of the assets to network exposure. The top panel focused on the banks dataset, the middle panel on the insurance companies dataset and the bottom panel on the industry portfolios dataset. The first column identifies the quantiles used to estimate the quantile-based network, and the second column indicates if a common factor was used (Y) or not used $(\mathrm{N})$ in the estimation of the causality networks. The results identify the four different networks which are optimally combined: baseline quantile causality; quantile-on-quantile causality; non-parametric quantile causality; Granger causality. A star identifies parameters significant at the $5 \%$ confidence level.

\begin{tabular}{cccc}
\hline Quantile & Factor & 2006-2008 & 2011-2015 \\
\hline \multicolumn{4}{c}{ 25 Banks } \\
\hline $10 \%$ & $\mathrm{~N}$ & $0.687^{*}$ & $0.727^{*}$ \\
$50 \%$ & $\mathrm{~N}$ & $0.756^{*}$ & $0.851^{*}$ \\
$90 \%$ & $\mathrm{~N}$ & $0.604^{*}$ & $0.774^{*}$ \\
$10 \%$ & $\mathrm{Y}$ & $0.656^{*}$ & $0.713^{*}$ \\
$50 \%$ & $\mathrm{Y}$ & $0.894^{*}$ & $0.886^{*}$ \\
$90 \%$ & $\mathrm{Y}$ & $0.639^{*}$ & $0.673^{*}$ \\
\hline \multicolumn{4}{c}{$\mathbf{2 5}$ Insurance Companies } \\
\hline $10 \%$ & $\mathrm{~N}$ & $0.497^{*}$ & $0.554^{*}$ \\
$50 \%$ & $\mathrm{~N}$ & $0.301^{*}$ & $0.613^{*}$ \\
$90 \%$ & $\mathrm{~N}$ & $0.469^{*}$ & $0.618^{*}$ \\
$10 \%$ & $\mathrm{Y}$ & $0.491^{*}$ & $0.581^{*}$ \\
$50 \%$ & $\mathrm{Y}$ & $0.472^{*}$ & $0.638^{*}$ \\
$90 \%$ & $\mathrm{Y}$ & $0.477^{*}$ & $0.564^{*}$ \\
\hline \multicolumn{5}{c}{} \\
\hline $10 \%$ & $\mathbf{4 8}$ Industry companies \\
$50 \%$ & $\mathrm{~N}$ & $-0.077^{*}$ & $0.460^{*}$ \\
$90 \%$ & $\mathrm{~N}$ & $-0.117^{*}$ & $0.025^{*}$ \\
$10 \%$ & $\mathrm{Y}$ & $-0.077^{*}$ & $0.324^{*}$ \\
$50 \%$ & $\mathrm{Y}$ & $0.175^{*}$ & $0.282^{*}$ \\
$90 \%$ & $\mathrm{Y}$ & $0.129^{*}$ & $0.123^{*}$ \\
\hline \multicolumn{5}{c}{$0.100^{*}$} & $0.252^{*}$ \\
\hline \multicolumn{4}{c}{}
\end{tabular}


Table H.30: The table reports residual correlation descriptive analyses for the Banks dataset. The first column identify the various models, while the second column indicates the number of networks used in the model. In the first column $Q(10 \%)$ identifies the use of a combination of causality networks from quantile regression (QB, Qo and $\mathrm{QN})$ at the $10 \%$ quantile, combined with the Granger causality network. Similarly, when the reference quantile is $50 \%$ or $90 \%$. With $G$ we denote the model using just the Granger casuality network, while the last line refers to the 4-factor CAPM. The table reports statistics for the residuals correlations: the minimum, maximum, the $10 \%$ quantile $q_{10}$, the median $q_{50}$, the $90 \%$ quantile and the number of elements of the correlation matrix lower than -0.1 .

\begin{tabular}{|c|c|c|c|c|c|c|c|c|}
\hline \multicolumn{9}{|c|}{ 2006-2008 } \\
\hline Model & $\begin{array}{c}\mathbf{N} \\
\text { Networks }\end{array}$ & Factor & Min & $\operatorname{Max}$ & q10 & q50 & q90 & $\begin{array}{c}\% \text { elements } \\
\leq-0.1\end{array}$ \\
\hline $10 \%$ & 4 & $\mathrm{~N}$ & -0.443 & 0.578 & -0.207 & 0.082 & 0.364 & $23.0 \%$ \\
\hline $50 \%$ & 4 & $\mathrm{~N}$ & -0.469 & 0.640 & -0.205 & 0.114 & 0.425 & $19.3 \%$ \\
\hline $90 \%$ & 4 & $\mathrm{~N}$ & -0.461 & 0.602 & -0.193 & 0.031 & 0.371 & $23.3 \%$ \\
\hline $10 \%$ & 4 & $\mathrm{Y}$ & -0.377 & 0.573 & -0.198 & 0.061 & 0.322 & $21.3 \%$ \\
\hline $50 \%$ & 4 & $\mathrm{Y}$ & -0.526 & 0.558 & -0.184 & 0.057 & 0.329 & $18.0 \%$ \\
\hline $90 \%$ & 4 & $\mathrm{Y}$ & -0.588 & 0.546 & -0.206 & 0.061 & 0.347 & $19.3 \%$ \\
\hline Granger & 1 & $\mathrm{~N}$ & -0.366 & 0.662 & -0.120 & 0.221 & 0.475 & $12.3 \%$ \\
\hline Granger & 1 & $\mathrm{Y}$ & -0.591 & 0.626 & -0.143 & 0.155 & 0.428 & $12.3 \%$ \\
\hline Multifactor & - & - & -0.358 & 0.678 & -0.108 & 0.257 & 0.502 & $10.7 \%$ \\
\hline \multicolumn{9}{|c|}{ 2011-2015 } \\
\hline Model & $\begin{array}{c}\mathrm{N} \\
\text { Networks }\end{array}$ & Factor & Min & $\operatorname{Max}$ & q10 & q50 & q90 & $\begin{array}{c}\% \text { elements } \\
\leq-0.1\end{array}$ \\
\hline $10 \%$ & 4 & $\mathrm{~N}$ & -0.450 & 0.394 & -0.146 & -0.019 & 0.186 & $20.0 \%$ \\
\hline $50 \%$ & 4 & $\mathrm{~N}$ & -0.416 & 0.594 & -0.145 & 0.091 & 0.308 & $15.7 \%$ \\
\hline $90 \%$ & 4 & $\mathrm{~N}$ & -0.639 & 0.444 & -0.136 & 0.005 & 0.169 & $17.7 \%$ \\
\hline $10 \%$ & 4 & $\mathrm{Y}$ & -0.320 & 0.462 & -0.154 & -0.013 & 0.181 & $22.0 \%$ \\
\hline $50 \%$ & 4 & $\mathrm{Y}$ & -0.423 & 0.560 & -0.168 & 0.071 & 0.290 & $17.7 \%$ \\
\hline $90 \%$ & 4 & $\mathrm{Y}$ & -0.369 & 0.482 & -0.156 & -0.005 & 0.166 & $23.0 \%$ \\
\hline Granger & 1 & $\mathrm{~N}$ & -0.468 & 0.598 & -0.052 & 0.169 & 0.369 & $6.7 \%$ \\
\hline Granger & 1 & $\mathrm{Y}$ & -0.472 & 0.598 & -0.053 & 0.167 & 0.369 & $6.7 \%$ \\
\hline Multifactor & - & - & -0.122 & 0.669 & 0.054 & 0.266 & 0.487 & $0.3 \%$ \\
\hline
\end{tabular}


Table H.31: The table reports residual correlation descriptive analyses for the Insurance Companies dataset. The first column identify the various models, while the second column indicates the number of networks used in the model. In the first column $Q(10 \%)$ identifies the use of a combination of causality networks from quantile regression (QB, Qo and QN) at the $10 \%$ quantile, combined with the Granger causality network. Similarly, when the reference quantile is $50 \%$ or $90 \%$. With $G$ we denote the model using just the Granger casuality network, while the last line refers to the 4-factor CAPM. The table reports statistics for the residuals correlations: the minimum, maximum, the $10 \%$ quantile $q_{10}$, the median $q_{50}$, the $90 \%$ quantile and the number of elements of the correlation matrix lower than -0.1 .

\begin{tabular}{|c|c|c|c|c|c|c|c|c|}
\hline \multicolumn{9}{|c|}{ 2006-2008 } \\
\hline Model & $\begin{array}{c}\mathbf{N} \\
\text { Networks }\end{array}$ & Factor & Min & $\operatorname{Max}$ & $q 10$ & q50 & q90 & $\begin{array}{c}\% \text { elements } \\
\leq-0.1\end{array}$ \\
\hline $\mathrm{Q}(10 \%)$ & 4 & $\mathrm{~N}$ & -0.499 & 0.809 & -0.261 & -0.004 & 0.294 & $31.7 \%$ \\
\hline $\mathrm{Q}(50 \%)$ & 4 & $\mathrm{~N}$ & -0.468 & 0.812 & -0.213 & 0.018 & 0.379 & $23.3 \%$ \\
\hline $\mathrm{Q}(90 \%)$ & 4 & $\mathrm{~N}$ & -0.477 & 0.804 & -0.257 & -0.008 & 0.315 & $30.7 \%$ \\
\hline $\mathrm{Q}(10 \%)$ & 4 & $\mathrm{Y}$ & -0.475 & 0.806 & -0.259 & -0.008 & 0.325 & $30.7 \%$ \\
\hline $\mathrm{Q}(50 \%)$ & 4 & $\mathrm{Y}$ & -0.461 & 0.810 & -0.243 & 0.009 & 0.346 & $26.3 \%$ \\
\hline $\mathrm{Q}(90 \%)$ & 4 & $\mathrm{Y}$ & -0.485 & 0.802 & -0.266 & -0.011 & 0.314 & $30.7 \%$ \\
\hline G & 1 & $\mathrm{~N}$ & -0.425 & 0.831 & -0.190 & 0.047 & 0.398 & $20.0 \%$ \\
\hline $\mathrm{G}$ & 1 & $\mathrm{Y}$ & -0.420 & 0.836 & -0.188 & 0.046 & 0.384 & $20.0 \%$ \\
\hline 4-F-CAPM & - & - & -0.370 & 0.847 & -0.149 & 0.089 & 0.422 & $14.0 \%$ \\
\hline \multicolumn{9}{|c|}{ 2011-2015 } \\
\hline Model & $\begin{array}{c}\mathrm{N} \\
\text { Networks }\end{array}$ & Factor & Min & $\operatorname{Max}$ & q10 & q50 & q90 & $\begin{array}{c}\% \text { elements } \\
\leq-0.1\end{array}$ \\
\hline $\mathrm{Q}(10 \%)$ & 4 & $\mathrm{~N}$ & -0.302 & 0.554 & -0.115 & 0.002 & 0.182 & $14.7 \%$ \\
\hline $\mathrm{Q}(50 \%)$ & 4 & $\mathrm{~N}$ & -0.293 & 0.636 & -0.135 & 0.032 & 0.235 & $15.3 \%$ \\
\hline $\mathrm{Q}(90 \%)$ & 4 & $\mathrm{~N}$ & -0.313 & 0.510 & -0.136 & 0.001 & 0.181 & $18.0 \%$ \\
\hline $\mathrm{Q}(10 \%)$ & 4 & $\mathrm{Y}$ & -0.314 & 0.593 & -0.152 & -0.002 & 0.193 & $21.0 \%$ \\
\hline $\mathrm{Q}(50 \%)$ & 4 & $\mathrm{Y}$ & -0.249 & 0.650 & -0.142 & 0.031 & 0.220 & $19.0 \%$ \\
\hline $\mathrm{Q}(90 \%)$ & 4 & $\mathrm{Y}$ & -0.292 & 0.545 & -0.149 & 0.006 & 0.194 & $22.0 \%$ \\
\hline G & 1 & $\mathrm{~N}$ & -0.109 & 0.658 & 0.001 & 0.116 & 0.322 & $1.0 \%$ \\
\hline $\mathrm{G}$ & 1 & $\mathrm{Y}$ & -0.103 & 0.658 & -0.011 & 0.120 & 0.318 & $1.0 \%$ \\
\hline 4-F-CAPM & - & - & -0.103 & 0.658 & 0.017 & 0.138 & 0.332 & $0.3 \%$ \\
\hline
\end{tabular}


Table H.32: The table reports residual correlation descriptive analyses for the Industry portfolios dataset. The first column identify the various models, while the second column indicates the number of networks used in the model. In the first column $Q(10 \%)$ identifies the use of a combination of causality networks from quantile regression (QB, Qo and QN) at the $10 \%$ quantile, combined with the Granger causality network. Similarly, when the reference quantile is 50\% or $90 \%$. With $G$ we denote the model using just the Granger casuality network, while the last line refers to the 4-factor CAPM. The table reports statistics for the residuals correlations: the minimum, maximum, the $10 \%$ quantile $q_{10}$, the median $q_{50}$, the $90 \%$ quantile and the number of elements of the correlation matrix lower than -0.1 .

\begin{tabular}{|c|c|c|c|c|c|c|c|c|}
\hline \multicolumn{9}{|c|}{ 2006-2008 } \\
\hline Model & $\begin{array}{c}\mathbf{N} \\
\text { Networks }\end{array}$ & Factor & Min & $\operatorname{Max}$ & $q 10$ & q50 & q90 & $\begin{array}{c}\% \text { elements } \\
\leq-0.1\end{array}$ \\
\hline $\mathrm{Q}(10 \%)$ & 4 & $\mathrm{~N}$ & -0.444 & 0.756 & -0.204 & 0.007 & 0.274 & $25.6 \%$ \\
\hline $\mathrm{Q}(50 \%)$ & 4 & $\mathrm{~N}$ & -0.449 & 0.757 & -0.198 & 0.014 & 0.276 & $24.9 \%$ \\
\hline $\mathrm{Q}(90 \%)$ & 4 & $\mathrm{~N}$ & -0.444 & 0.756 & -0.204 & 0.007 & 0.274 & $25.6 \%$ \\
\hline $\mathrm{Q}(10 \%)$ & 4 & $\mathrm{Y}$ & -0.476 & 0.731 & -0.226 & -0.013 & 0.253 & $28.2 \%$ \\
\hline $\mathrm{Q}(50 \%)$ & 4 & $\mathrm{Y}$ & -0.494 & 0.733 & -0.228 & -0.007 & 0.265 & $28.2 \%$ \\
\hline $\mathrm{Q}(90 \%)$ & 4 & $\mathrm{Y}$ & -0.463 & 0.728 & -0.227 & -0.005 & 0.261 & $28.5 \%$ \\
\hline G & 1 & $\mathrm{~N}$ & -0.444 & 0.756 & -0.204 & 0.007 & 0.274 & $25.6 \%$ \\
\hline $\mathrm{G}$ & 1 & $\mathrm{Y}$ & -0.415 & 0.752 & -0.203 & 0.009 & 0.273 & $25.4 \%$ \\
\hline 4-F-CAPM & - & - & -0.486 & 0.733 & -0.219 & 0.003 & 0.267 & $27.1 \%$ \\
\hline \multicolumn{9}{|c|}{ 2011-2015 } \\
\hline Model & $\begin{array}{c}\mathrm{N} \\
\text { Networks }\end{array}$ & Factor & Min & $\operatorname{Max}$ & q10 & q50 & q90 & $\begin{array}{c}\% \text { elements } \\
\leq-0.1\end{array}$ \\
\hline $\mathrm{Q}(10 \%)$ & 4 & $\mathrm{~N}$ & -0.434 & 0.560 & -0.161 & -0.015 & 0.154 & $24.3 \%$ \\
\hline $\mathrm{Q}(50 \%)$ & 4 & $\mathrm{~N}$ & -0.450 & 0.588 & -0.148 & 0.007 & 0.204 & $18.8 \%$ \\
\hline $\mathrm{Q}(90 \%)$ & 4 & $\mathrm{~N}$ & -0.424 & 0.551 & -0.159 & -0.010 & 0.170 & $23.5 \%$ \\
\hline $\mathrm{Q}(10 \%)$ & 4 & $\mathrm{Y}$ & -0.431 & 0.564 & -0.166 & -0.010 & 0.179 & $23.0 \%$ \\
\hline $\mathrm{Q}(50 \%)$ & 4 & $\mathrm{Y}$ & -0.461 & 0.589 & -0.152 & 0.007 & 0.191 & $19.1 \%$ \\
\hline $\mathrm{Q}(90 \%)$ & 4 & $\mathrm{Y}$ & -0.432 & 0.567 & -0.166 & -0.005 & 0.183 & $22.6 \%$ \\
\hline G & 1 & $\mathrm{~N}$ & -0.467 & 0.589 & -0.144 & 0.009 & 0.208 & $18.4 \%$ \\
\hline $\mathrm{G}$ & 1 & $\mathrm{Y}$ & -0.445 & 0.592 & -0.148 & 0.008 & 0.208 & $18.4 \%$ \\
\hline 4-F-CAPM & - & - & -0.456 & 0.592 & -0.147 & 0.007 & 0.208 & $18.4 \%$ \\
\hline
\end{tabular}




\section{Estimated Parameters}

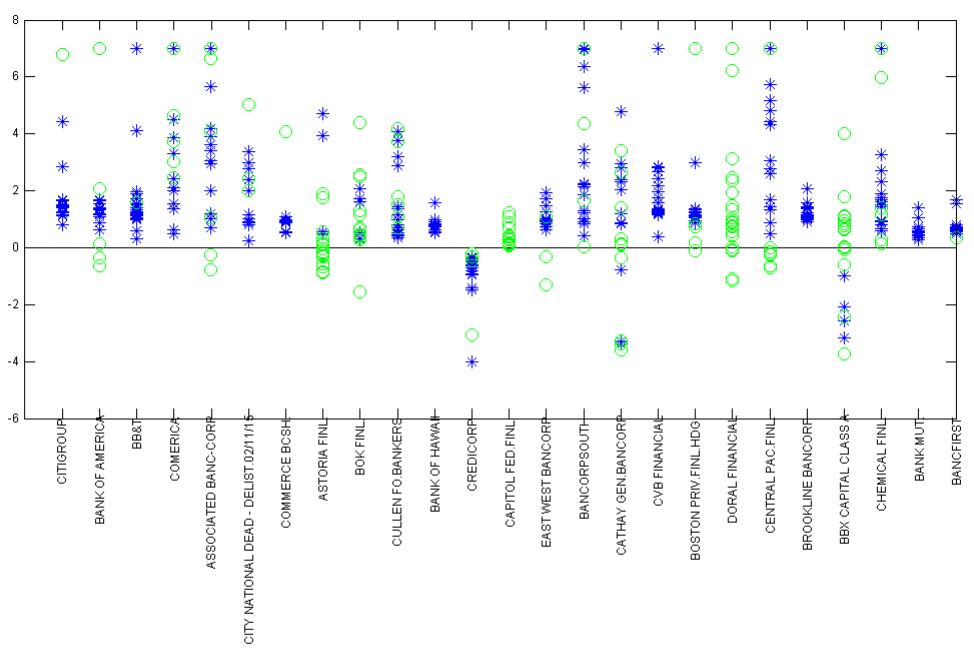

(a) $2006-2008$

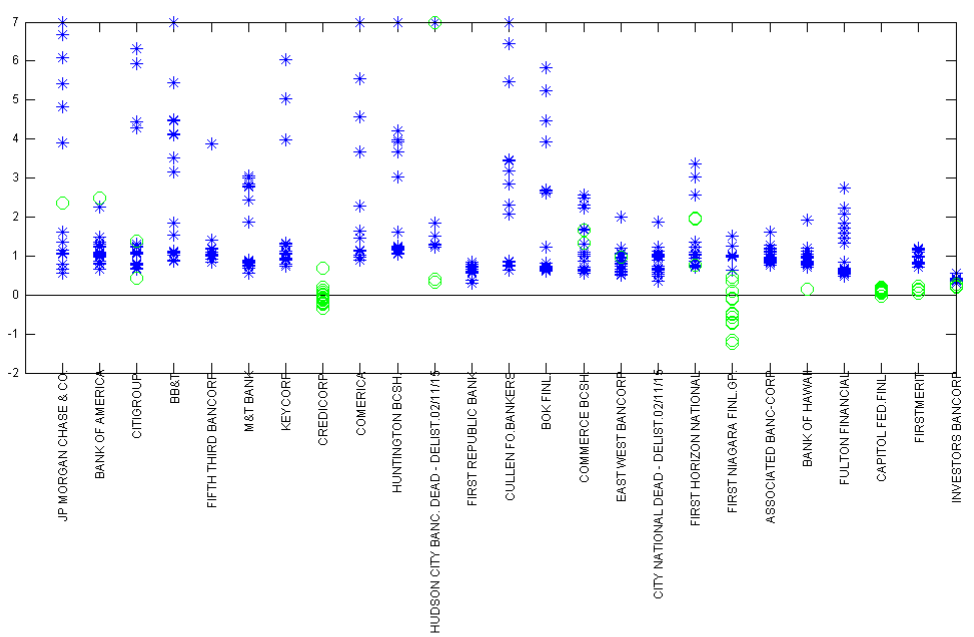

(b) $2011-2015$

Figure I.27: This figure exhibits the $\rho^{\prime} s$ coefficients signficant (blue asterisks ${ }^{\prime}{ }^{\prime}$ ) and not significant (green circle 'o') by using different resulting networks obtained combining different causality methodologies. The $\rho^{\prime} s$ parameters are relative to first 25 banks monthly returns ordered for market capitalization. Panel a) reports the coefficients relative to the period 2006-2008. Panel b) reports the coefficients relative to the period $2011-2015$. 


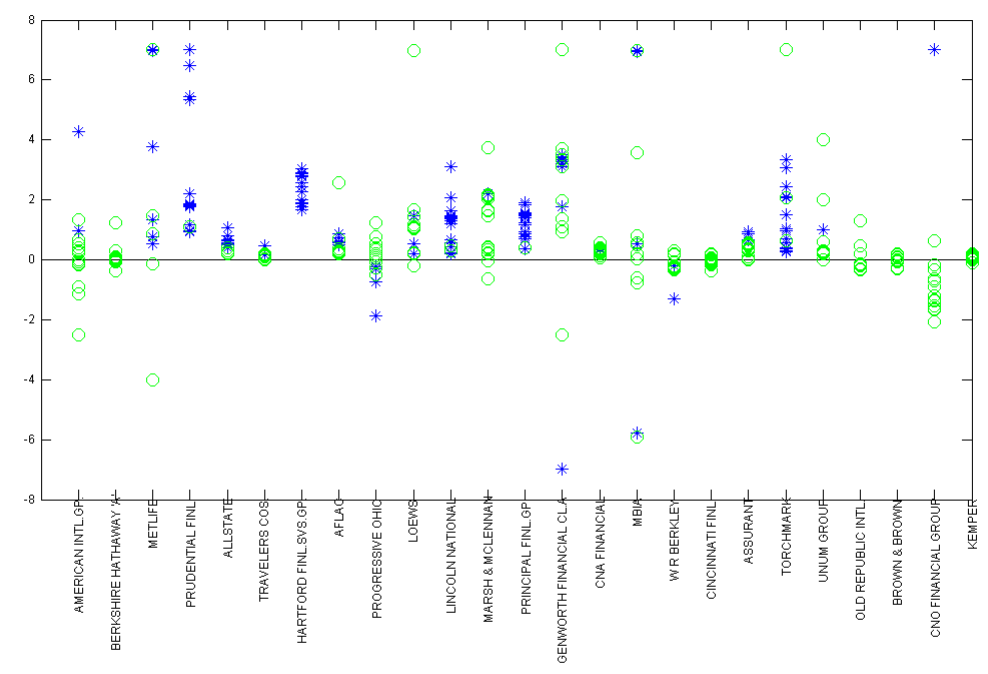

(a) $2006-2008$

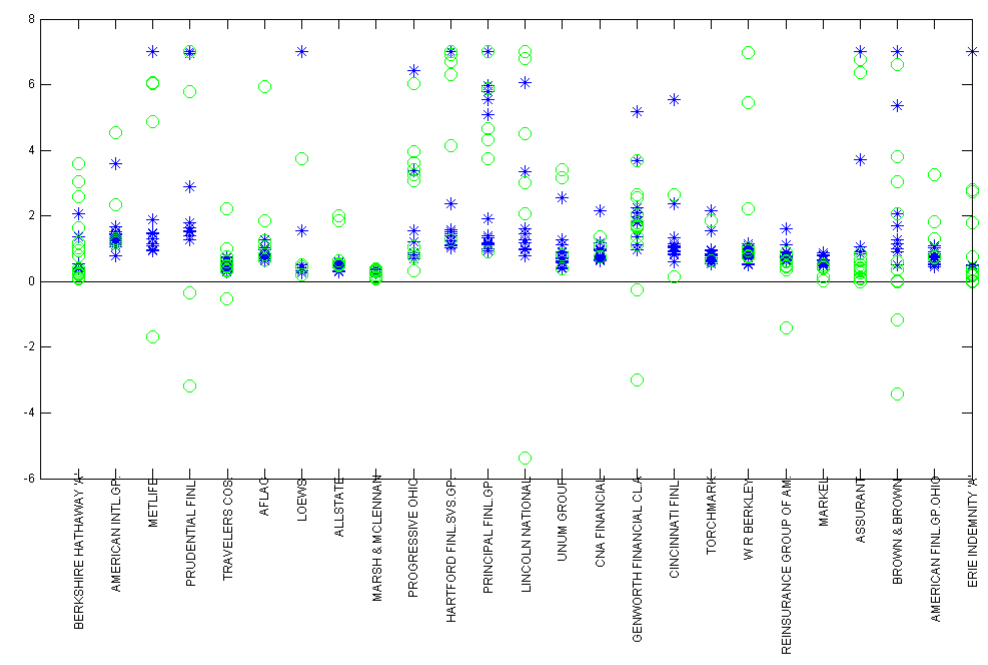

(b) $2011-2015$

Figure I.28: This figure exhibits the $\rho^{\prime} s$ coefficients signficant (blue asterisks '*') and not significant (green circle 'o') by using different resulting networks obtained combining different causality methodologies. The $\rho^{\prime} s$ parameters are relative to first 25 Insurers monthly returns ordered for market capitalization. Panel a) reports the coefficients relative to the period 2006-2008. Panel b) reports the coefficients relative to the period $2011-2015$. 


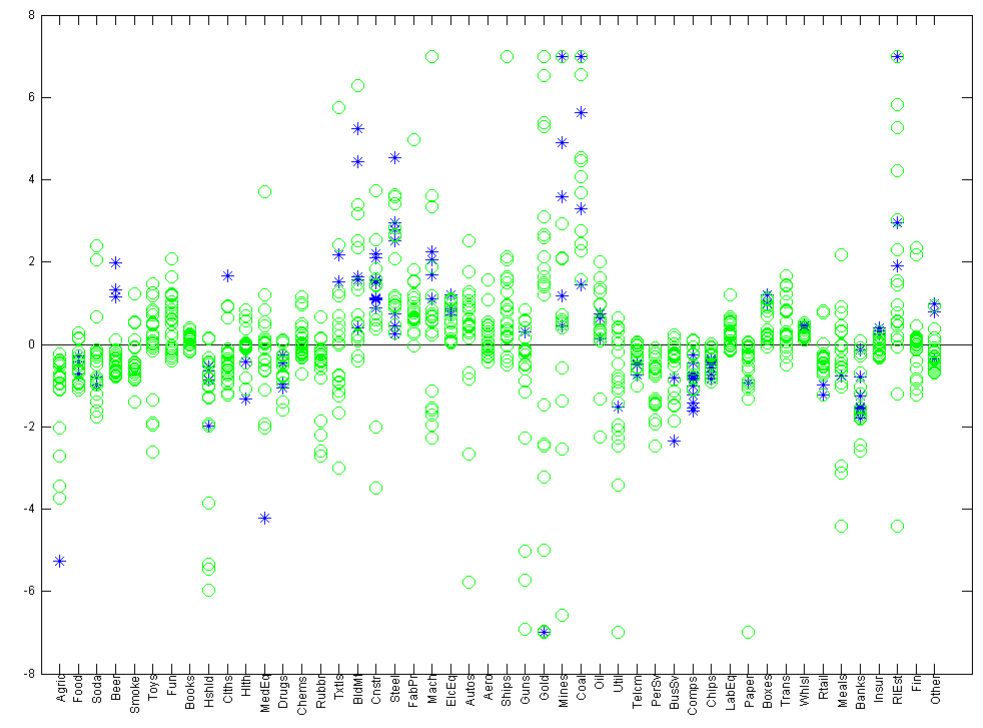

(a) $2006-2008$

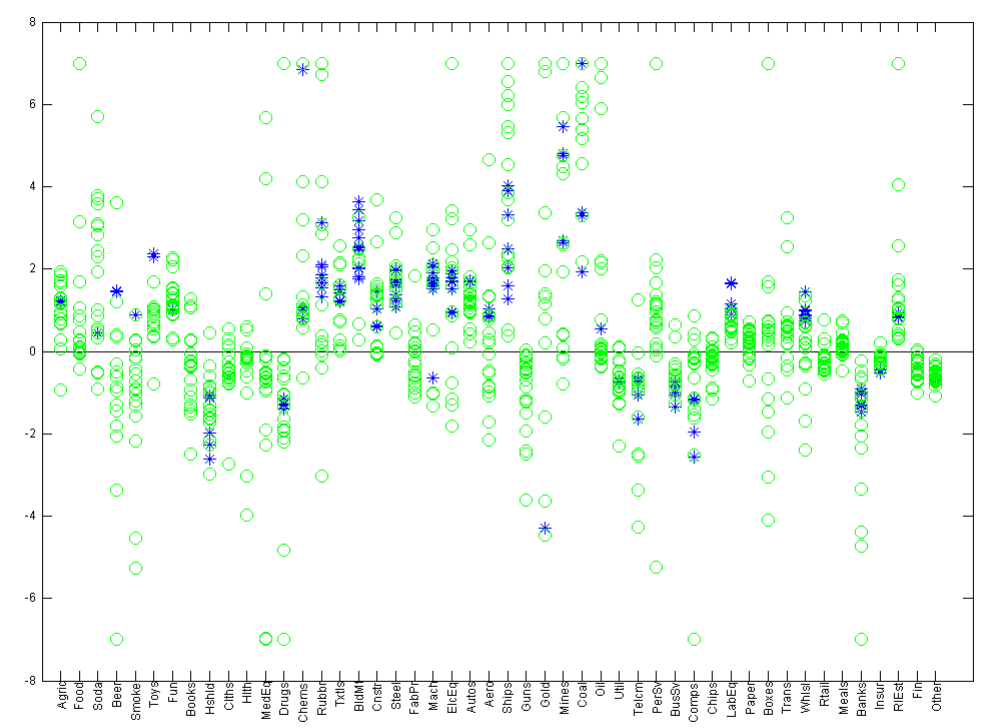

(b) $2011-2015$

Figure I.29: This figure exhibits the $\rho^{\prime} s$ coefficients signficant (blue asterisks ${ }^{\prime}{ }^{\prime}$ ) and not significant (green circle 'o') by using different resulting networks obtained combining different causality methodologies. The $\rho^{\prime} s$ parameters are relative to 48 Fama and French industry portfolios monthly returns ordered for market capitalization. Panel a) reports the coefficients relative to the period $2006-2008$. Panel b) reports the coefficients relative to the period $2011-2015$. 


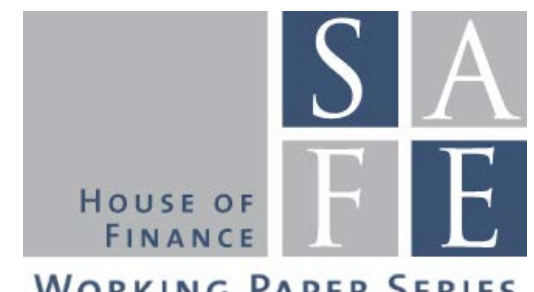

Working PAPER SERIES

\section{Recent Issues}

No. 164 Raimond Maurer, Olivia S. Mitchell, Ralph Rogalla, Tatjana Schimetschek

No. 163 Giuliano Curatola, Michael Donadelli, Patrick Grüning

No. 162 Gabriele Camera, Alessandro Gioffré

No. 161 Tobin Hanspal

No. 160 Domenico Rocco Cambrea, Stefano Colonnello, Giuliano Curatola, Giulia Fantini

No. 159 Monica Billio, Michael Donadelli, Antonio Paradiso, Max Riedel

No. 158 Michael Donadelli, Renatas Kizys, Max Riedel

No. 157 Steffen Meyer, Linda Urban, Sophie Ahlswede

No. 156 Reint Gropp, Thomas Mosk, Steven Ongena, Carlo Wix

No. 155 Vahid Saadi

No. 154 Brigitte Haar

No. 153 Julia Hirsch, Uwe Walz

No. 152 Viral Acharya, Tim Eisert, Christian Eufinger, Christian Hirsch
Optimal Social Security Claiming Behavior under Lump Sum Incentives: Theory and Evidence

Technology Trade with Asymmetric Tax Regimes and Heterogeneous Labor Markets: Implications for Macro Quantities and Asset Prices

Asymmetric Social Norms

The Effect of Personal Financing Disruptions on Entrepreneurship

Abandon Ship: Inside Debt and Risk-Taking Incentives in Bad Times

Which Market Integration Measure?

Globally Dangerous Diseases: Bad News for Main Street, Good News for Wall Street?

Does feedback on personal investment success help?

Bank Response To Higher Capital Requirements: Evidence From A QuasiNatural Experiment

Mortgage Supply and the US Housing Boom: The Role of the Community Reinvestment Act

Shareholder Wealth vs. Stakeholder interests? Evidence from Code Compliance under the German Corporate Governance Code

The Financing Dynamics of Newly Founded Firms

Whatever it Takes: The Real Effects of Unconventional Monetary Policy 